\title{
Dimensionen religiöser Erziehung muslimischer Kinder in Niedersachsen
}

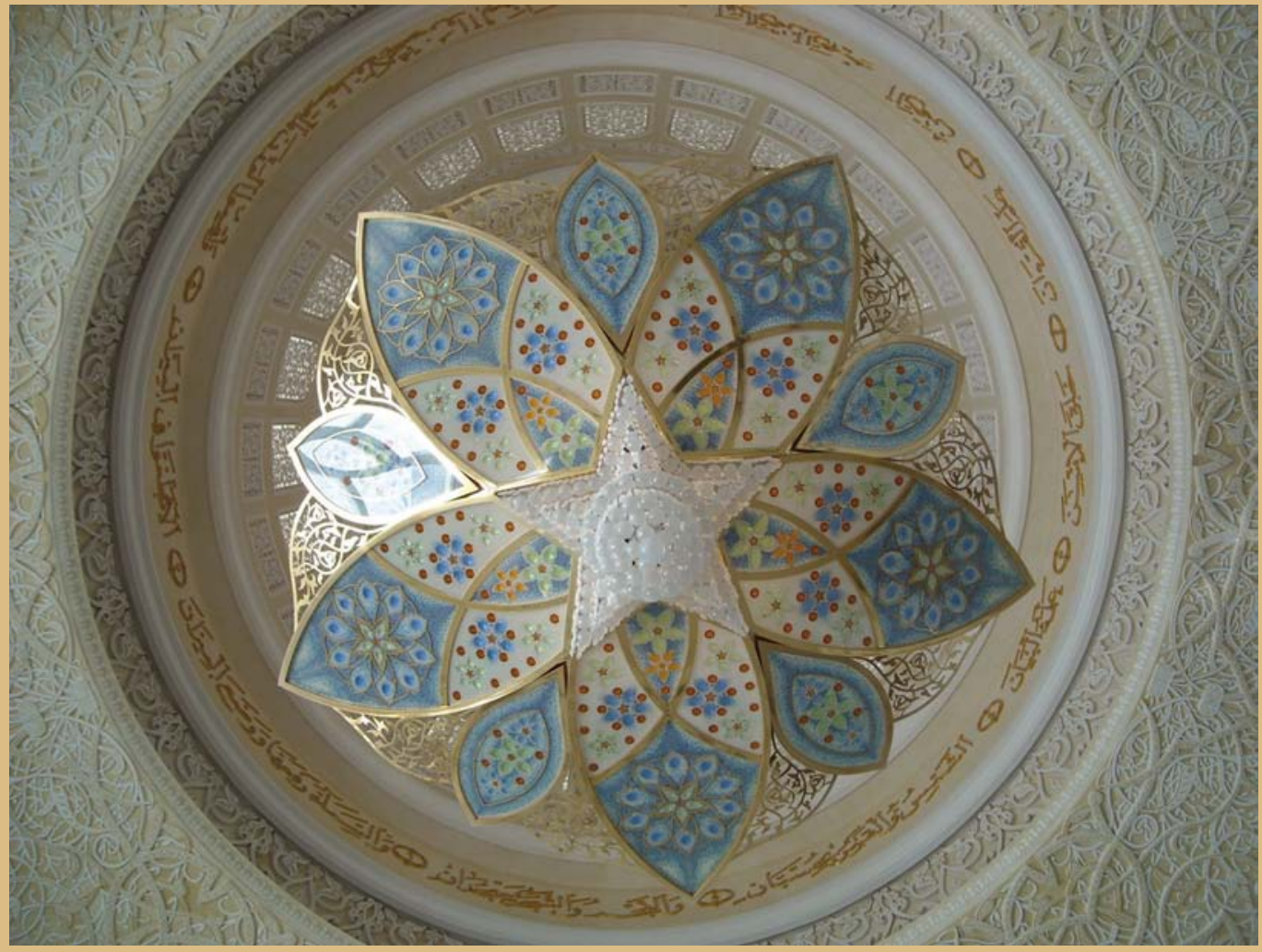



Katja Koch und Kinan Darwisch

Dimensionen religiöser Erziehung muslimischer Kinder in Niedersachsen

This work is licensed under the

Creative Commons License 3.0 "by-nd", allowing you to download, distribute and print the document in a few copies for private or educational use, given that the document stays unchanged and the creator is mentioned. You are not allowed to sell copies of the free version.

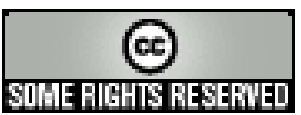


erschienen in der Reihe der Universitätsdrucke

im Universitätsverlag Göttingen 2010 
Katja Koch und

Kinan Darwisch (Hg.)

Dimensionen religiöser Erziehung muslimischer Kinder in Niedersachsen

Ergebnisse der Tagung des

Pädagogischen Seminars der

Georg-August-Universität Göttingen in Kooperation mit der

SCHURA Niedersachsen,

Landesverband der Muslime in

Niedersachsen e.V.

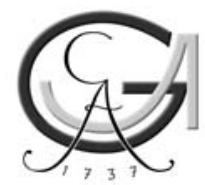

Universitätsverlag Göttingen 2010 


\section{Bibliographische Information der Deutschen Nationalbibliothek}

Die Deutsche Nationalbibliothek verzeichnet diese Publikation in der Deutschen Nationalbibliographie; detaillierte bibliographische Daten sind im Internet über $<$ http://dnb.ddb.de $>$ abrufbar.

\section{Kontaket}

Prof. Dr. Katja Koch

Pädagogisches Seminar der Georg-August-Universität Göttingen

Baurat-Gerber-Str. 4-6

37073 Göttingen

e-mail:kkoch@gwdg.de

Dieses Buch ist auch als freie Onlineversion über die Homepage des Verlags sowie über den OPAC der Niedersächsischen Staats- und Universitätsbibliothek (http://www.sub.uni-goettingen.de) erreichbar und darf gelesen, heruntergeladen sowie als Privatkopie ausgedruckt werden. Es gelten die Lizenzbestimmungen der Onlineversion. Es ist nicht gestattet, Kopien oder gedruckte Fassungen der freien Onlineversion zu veräußern.

Satz und Layout: Kinan Darwisch Umschlaggestaltung: Franziska Lorenz Titelabbildung: (C) Janine Grimmig / PIXELIO' www.pixelio.de 
Katja Koch \& Kinan Darwisch: Zur Einführung in diesen Band ................................. 4

Avni Altiner: Dimensionen religiöser Erziehung muslimischer Kinder in

Niedersachsen - Grußwort des Vorsitzenden der Schura Niedersachsen............. 8

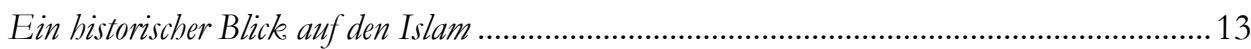

Gerdien Jonker: Den Islam erzählen. Interpretationen der muslimisch geprägten

Welt in europäischen Schulbüchern ...................................................................... 14

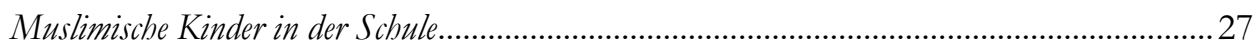

Katja Koch: Migrantenkinder im deutschen Schulwesen............................................28

Nazife Toklu: Individuelle und institutionelle Diskriminierung muslimischer Kinder in deutschen Schulen ................................................................................... 42

Auf dem Weg zum Islamischen Religionsunterricht ............................................................. 51

Heidemarie Ballasch: Schulversuch „Islamischer Religionsunterricht“ in

Niedersachsen. Auf dem Weg zum Islamischen Religionsunterricht als ordentliches Unterrichtsfach.

Kinan Darwisch: Islamischer Religionsunterricht in Deutschland und seine Entwicklung in Niedersachsen

Haci-Halil Uslucan: Islamischer Religionsunterricht in der Grundschule: Ein Modellversuch zur Nachahmung? .........................................................................74

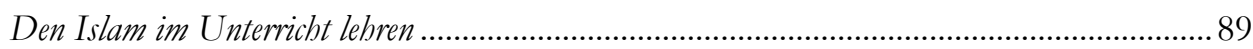

Ali Özgur Özdil: Lehrtradition, Vorbild im Glauben versus neue muslimische Fachdidaktik und Moscheepädagogik

Katja Koch: Was sollen Lehrerinnen und Lehrer für den islamischen Religionsunterricht können? 100

Bemerkungen zum Scbluss....

Betram Schmitz, Kinan Darwisch: Zwischen Würdigung und Verantwortung Perspektiven zum Islamunterricht an deutschen Schulen. 
Firouz Vladi: Was nötig ist: Mit Vertrauen auf Klassenfahrt, interkulturelle Kompetenz für Schulen und Jugendeinrichtungen sowie einige Anmerkungen zu einer korrekten islamischen Terminologie.

Die Autorinnen und Autoren ............................................................................................. 131 
Vor- und Grußworte 


\section{Katja Koch \& Kinan Darwisch: Zur Einführung in diesen Band}

„Können Sie es sich vorstellen gemeinsam mit der Schura Niedersachsen eine Tagung zum Islamischen Religionsunterricht zu organisieren?" Diese Frage stellte mir Kinan Darwisch vor einiger Zeit in meinem Büro. Nach kurzer Bedenkzeit sagte ich zu, nicht nur, weil das Thema damals in „der Luft lag“, sondern auch, weil ich neugierig war, wie jene den Schulversuch sehen, für deren Kinder er gedacht war. Was halten Muslime vom niedersächsischen Schulversuch? Was sind ihre Interessen in diesem Zusammenhang und welche Veränderungen wünschen sie sich? Gleichzeitig fand ich, dass sich die Erziehungswissenschaft allgemein, und die Schulpädagogik im Besonderen, bisher zu wenig mit diesem Thema beschäftigt hat.

Der Zeitpunkt für die Tagung war gut gewählt: Der Islamische Religionsunterricht lief seit einigen Jahren, es gab eine erste wissenschaftliche Evaluation und beide Seiten, sowohl die Bildungsadministration als auch die Muslime konnten erste Erfahrungen sammeln. In den vorbereitenden Gesprächen mit der Schura Niedersachsen e.V., hier vor allem mit Herrn Vladi und Herrn Al-Dalati, wurde schnell deutlich, dass ein gemeinsames Ziel der Tagung eine erste Bestandsaufnahme des Islamischen Religionsunterrichts und ein offener Dialog über seine weitere Gestalt sein sollte. Das Programm der Tagung, die im übrigen mit über 200 Teilnehmerinnen und Teilnehmern sehr gut besucht war und aufgrund der 
vielen interessanten sowie engagierten Diskussionen auch zwei Stunden länger dauerte als geplant, spiegelt das Bemühen um diesen Dialog: Die von uns als relevant eingeschätzten Themen wurden mit jeweils zwei Referentinnen und Referenten besetzt, die wiederum unterschiedliche Facetten der Thematik beleuchteten und verschiedene Sichtweisen einbrachten. Für den hier vorliegenden Tagungsband haben wir diese Struktur weitgehend beibehalten, die Beiträge wurden thematisch zusammengefasst und um einen weiteren, auf der Tagung nicht gehaltenen Vortrag von Katja Koch sowie um ein gemeinsames Nachwort von Bernhardt Schmitz und Kinan Darwisch ergänzt.

Nach unserer Einführung beginnt der Tagungsband mit einem Grußwort des Vorsitzenden der Schura Niedersachen e.V., Avni Altiner, der aus seiner muslimischen Sicht in Niedersachsen wichtige Dimensionen religiöser Erziehung hervorhebt. Gerdien Jonker vom Georg-Eckert-Institut in Braunschweig zeigt die historische Dimension des Umgangs mit dem Islam in Westeuropa auf. Sie verdeutlicht mit Blick auf die Schulbücher, dass sich dort zwar althergebrachte Vorbehalte finden, diese jedoch nicht als Ausdruck einer allgemeinen Islamfeindlichkeit zu werten sind.

Mit der Situation von muslimischen Kindern in der Schule beschäftigen sich die Beiträge von Katja Koch und Nazife Toklu. Nazife Toklu berichtet aus ihrer Erfahrung als Mitarbeiterin in unterschiedlichen Integrationsprojekten von alltäglichen Formen der institutionellen Diskriminierung muslimischer Schülerinnen und Schüler. Der für die Veröffentlichung neu aufgenommene Beitrag von Katja Koch ordnet diese Erfahrungen in die schulpädagogische Diskussion um die Benachteiligung von Migrantenkindern im deutschen Bildungswesen ein. Aufgezeigt werden aktuelle Befunde zur Bildungsbeteiligung von Kindern mit Migrationshintergrund und mögliche Ursachen hierfür.

Der vierte Teil des Tagungsbandes ist dem Schulversuch „Islamischer Religionsunterricht", seiner Verwirklichung, seiner rechtlichen Probleme und seiner Evaluation gewidmet. Heidemarie Ballasch, die im Niedersächsischen Kultusministerium den Schulversuch von Beginn an federführend begleitet hat, beschreibt nicht nur, welche Schritte von Seiten der Bildungsadministration unternommen wurden, sondern führt auch den Prozess des Aushandelns am Runden Tisch vor Augen. Kinan Darwisch, Georg-August-Universität Göttingen, führt aus, welche juristischen Fallstricke und Schwierigkeiten bisher eine flächendeckende Einführung des Schulversuchs verhindern und wie diese beseitigt werden können. Dass dieser Unterricht bereits jetzt von den Schülerinnen und Schülern angenommen wird und tatsächlich einen Beitrag zur Integration leisten kann, zeigen die Untersuchungsergebnisse von denen Haci-Hali Uslucan berichtet.

Die Beiträge von Ali Özgür Özdil und Katja Koch stellen schließlich die didaktische Dimension des Islamischen Religionsunterrichts heraus. Ali Özgür Özdil benennt in seinem Beitrag einige aus muslimischer Sicht wichtige Leerstellen der bisherigen didaktischen Ausrichtung des Islamischen Religionsunterrichts und 
fordert Lehrerinnen und Lehrer, die für ihre Schülerinnen und Schüler vor allem Vorbilder im Glauben sind. Katja Koch betont in ihrem Beitrag, dass die Lehrerinnen und Lehrer für den Islamischen Religionsunterricht vor allem als Fachleute für guten Unterricht zu betrachten seien und daher dieselben Anforderungen erfüllen müssten wie andere Lehrerinnen und Lehrer auch. „Guter“ Islamischer Religionsunterricht brauche Lehrerinnen und Lehrer, die über eine entsprechend fachwissenschaftliche, didaktische und diagnostische Expertise verfügen.

Unser Tagungsband endet mit einer Nachlese. Bernhard Schmitz und Kinan Darwisch reflektieren die bisher erfolgten Maßnahmen und loten zukünftige Perspektiven aus. Firouz Vladi stellt aus Sicht der Schura Niedersachsen e.V. kritische Punkte heraus und fordert u.a. interkulturelle Kompetenzen für Lehrerinnen und Lehrer.

Als Herausgeber haben wir uns bei der Redaktion der Beiträge die Freiheit genommen, einzelne Passagen stilistisch zu korrigieren. Inhaltlich haben wir die Beiträge nicht verändert, so dass die Meinungen und Ansichten der jeweiligen Autorinnen und Autoren noch deutlich hervortreten. Manchen Aussagen stimmen wir zu, andere sehen wir deutlich kritischer. Wir hoffen, dass die Beiträge dieses Bandes Anlass zur produktiven Auseinandersetzung mit dem Islamischen Religionsunterricht geben und die Leserinnen und Leser die Chance nutzen, sich einer anderen Sichtweise anzunähern, die jeweiligen Argumente wahrzunehmen und produktiv miteinander darüber zu diskutieren.

Wir selbst sind davon überzeugt, dass die Einrichtung eines Islamischen Religionsunterrichts, wenn auch vorerst nur als Schulversuch vor allem „Normalität“ und Wertschätzung symbolisiert. Eine flächendeckende Einführung ist wünschenswert und erscheint uns als längst überfälliger Schritt. Dass hierbei der Islamische Religionsunterricht denselben fachlichen und fachdidaktischen Standards genügen muss, wie anderer Unterricht auch, halten wir für selbstverständlich. Dies bedeutet, dass es im Islamischen Religionsunterricht, wie in jedem Religionsunterricht, nicht vorrangig darum gehen kann, die Religiosität der Schülerinnen und Schüler zu fördern und auf die Einhaltung religiöser Praxen zu drängen, sondern dass die Reflexion der eigenen Religiosität (oder Nichtreligiosität) Gegenstand des Unterrichts sein sollte. In diesem Sinne wünschen wir uns eine akademische Ausbildung zukünftiger Lehrerinnen und Lehrer für den Islamischen Religionsunterricht an den Universitäten und eine Integration des Faches in den bisherigen schulischen Fächerkanon. Hierbei soll auch kurz angemerkt sein, dass eine universitäre Imam-Ausbildung in diesem Kontext, wie sie im niedersächsischen Osnabrück angedacht ist, von höchstem integrations-politischen und -pädagogischen Interesse ist sowie den in Niedersachsen mutig eingeschlagenen Weg letztlich noch mehr stärken könnte.

Wir möchten an dieser Stelle nochmals die Gelegenheit nutzen, um uns vor allem bei der Schura Niedersachsen e.V. für die gute inhaltliche Kooperation, die finanzielle Unterstützung bei der Ausrichtung der Tagung und der Drucklegung des 
Tagungsbandes zu bedanken. Dem Pädagogischen Seminar Göttingen danken wir für die Bereitstellung der Räume, der Göttinger Akademie der Wissenschaften für ihren finanziellen Zuschuss zur Tagung und der Moscheegemeinde Göttingen für die Verpflegung der Tagungsgäste. Dem etwas verspäteten Zeitpunkt der Veröffentlichung des vorliegenden Tagungsbandes liegen verschiedenste Verzögerungen zu Grunde. Dennoch haben die Verfasser und die Schura Niedersachsen e.V. das gemeinsame Ziel einer Veröffentlichung nie aufgegeben. Das deutsche Sprichwort „W as lange währt, wird endlich gut" möge daher sowohl auf den Tagungsband als auch auf den Islamischen Religionsunterricht in Niedersachsen (und Deutschland) zutreffen. 


\section{Avni Altiner: Dimensionen religiöser Erziehung muslimischer Kinder in Niedersachsen - Grußwort des Vorsitzenden der Schura Niedersachsen}

Liebe Geschwister im Islam, sehr geehrte Damen und Herren,

"Assalamu aleikum wa rahmatullah wa barakatub. ${ }^{\text {" }}$

Muslim sein ist keinem in die Wiege gelegt, man muss es im Lauf des Lebens erst erlernen. Diese uralte Einsicht gilt bis heute. Wer sich auf den Weg macht und sucht, der kann ankommen. Wer meint, schon angekommen zu sein, woher hat er Gewissheit, nicht am Ende in einer Sackgasse zustehen? Wer schon als Kind im Islam aufgewachsen ist, unter der Anleitung und dem Vorbild von Eltern mit Iman $^{2}$ und Taqwa ${ }^{3}$, der steht als Jugendlicher und Erwachsener dennoch vor der Aufgabe, das Muslim sein immer weiter zu erlernen, zu verfeinern. Und wer sich erst im Jugend- oder Erwachsenenalter für den Islam entscheidet, sei es als Konvertit oder sei es aus einem säkularen oder gar agnostischen Elternhaus ursprünglich muslimischer Abkunft, wird den muslimischen Glauben im Vorfeld kennen-

\footnotetext{
1 Islamische Grußformel: „Der Frieden Sei mit Euch sowie Gottes Barmherzigkeit und sein Segen“ 2 Arabisch: Glauben.

3 Arabisch: Gottesfurcht
} 
lernen und einüben. Es geht darum, sich Allahs Gnade der Rechteleitung mit Kopf, Herz und Hand zu Eigen zu machen und im Leben zu verwirklichen. Dies ist ein Prozess, der lebenslanges Lernen erfordert.,

Für die Schura Niedersachsen zeichnen sich vier Lern- und Sozialisationsinstanzen $\mathrm{ab}$, in denen der Islam vermittelt und eingeübt werden kann:

Ein erster lebensprägender Ort ist die Familie, in der Eltern ihre Kinder religiös erziehen. Hier können Kinder vertrauensvolles Gebet, as-salah („die an das verborgene Glauben und das Gebet verrichten“, Qur'an ${ }^{4}$ 2/2) und du'a, Respekt, Anstand und die Sittlichkeit des Lebens zwischen den Generationen und Geschlechtern erfahren und die wunderbaren qur'anischen Geschichten von Allah und den Menschen hören. Hier werden Eltern zu den ersten Zeugen des Glaubens, wenn sie ihre Kinder altersgemäß in die muslimische Orthopraxie und Tradition einführen, am Freitag zum Cum'a mitnehmen und ihnen Taqwa und Ethik, Adhab $^{5}$ vor leben. Der Prophet (s.a.s.) ${ }^{6}$ sagte:

„Die besten Eltern sind die Eltern, die ibren Kindern eine religiöse Erziehung zuteil kommen lassen und ibnen Ethik früh vermitteln" (Hadis) $)^{\text {. }}$

Eine zweite Instanz sind die Moscheen, in denen Kinder und Jugendliche aufwachsen, in der Jugendgruppe der Moschee Gemeinschaft erfahren, sich im Adhan ${ }^{8}$ üben, Festgebete und Trauertage mitgestalten und erleben des weiteren in der Jugendgruppe der Gleichaltrigen ihre Identität ausbilden und sich sozial engagieren.

„In der Tat, wer auch immer sich Allah bingibt und Gutes tut, der hat seinen Lohn bei seinem Herrn; und keine Furcht kommt über sie und Sie werden nicht traurig sein, (Qur'an) 2/112).

Wir wissen jedoch alle, dass nicht überall solche ideale Bedingungen herrschen, weder in Familien noch in den Moscheevereinen. Viele Elternpaare fühlen sich überfordert, ihr Kind im Islam zu erziehen, weil sie unsicher sind oder einer religiösen Erziehung ihres Kindes skeptisch gegenüberstehen. „Seine Religion soll das Kind später einmal selbst wählen“, hört man gelegentlich. Wie aber soll man sich für etwas entscheiden können, was man gar nicht kennt? Würde eine Mutter oder ein Vater jemals sagen: „Wir wissen nicht, ob unser Kind geliebt werden möchte, dazu soll es sich später selbst entscheiden"? Nein, in der Erziehung wollen Eltern

\footnotetext{
4 Es wird vorliegend die der arabischen Aussprache näherliegende Ausschreibung „Qur’an“ für das heilige Buch der Muslime verwendet und nicht wie meist im deutschsprachigen Raum oft noch üblich „Koran“

5 arabisch: gutes Benehmen

6 Abkürzung für arabisch: „Salla-llahu aleihi wasallam“. Dies ist der gebräuchliche Segens- und Friedenswunsch, den Muslime dem Propheten Mohammed wünschen. Wörtlich bedeutet er: "Allahs Segen und Frieden auf ihm.“

7 Hadis = Überlieferungen des Propheten.

8 Gebetsruf
} 
vielmehr alles weitergeben, was ihnen im Leben wichtig geworden ist. Nicht anders darf es mit der Liebe zu Allah sein. Daher sollten Eltern hierbei die Unterstützung erfahren, die sie benötigen, um ihre Kinder religiös zu erziehen.

Die dritte Sozialisationsinstanz wird aus dem Zitat des ehrwürdige Propheten deutlich, in welchem er sagt: „Kinder, die von ihren Eltern nicht erzogen werden, werden von der Gesellschaft erzogen.". Da wir als Muslime in der Diaspora leben und in der Mehrheitsgesellschaft eine Minderheit sind, ist umso wichtiger, dass muslimische Kinder die Chance erhalten, neben der religiösen Erziehung im Elternhaus und den Moscheen auch am islamischen Religionsunterricht in der Schule teilzunehmen.

Die vierte wichtige Instanz der Begegnung mit dem Islam ist somit der schulische Religionsunterricht. Schule gewinnt heute als Lebensraum für Kinder und Jugendliche zunehmend an Bedeutung und der islamische Religionsunterricht ist in diesem Rahmen ein wichtiger Lernort des Glaubens, vor allem dann, wenn wie oben erwähnt, viele Kinder und Jugendliche in ihren Familien bisher keinen $\mathrm{Zu}$ gang zum gelebten Islam erfahren haben. Im Wissen darum haben wir uns als Schura Niedersachsen seit etwa 6 Jahren für die Einführung des islamischen Religionsunterrichts eingesetzt.

Als Fachleute vor einigen Jahren nach der Akzeptanz des Religionsunterrichts in der Schule fragten, erlebten sie eine Überraschung. Schülerinnen und Schüler, so fanden sie heraus, sind keineswegs desinteressiert, vielmehr ist der Religionsunterricht für die überwältigende Mehrheit ein „beliebtes Fach“, das sie als wichtig für ihr Leben einstufen. Dies sollte auch für muslimische Kinder und Jugendliche möglich sein. Der Religionsunterricht allgemein und damit auch der islamische Religionsunterricht vermittelt ein lebensdeutsames Glaubenswissen, das das Verantwortungsbewusstsein und die Urteilsfähigkeit des Einzelnen fördert. Im islamischen Religionsunterricht lernen die Schülerinnen und Schüler den Qur'an nach seinen Inhalten kennen, angefangen vom Bericht über Allahs wundervolle und schöne Schöpfung bis hin zu den lebenspraktischen Vorbildern unseres Propheten Muhammad (s.a.s.) und seiner Familie und Gefährten. Auch mit dem Wirken der ihm vorangegangenen Propheten unter den Menschen und Völkern macht sie der islamische Religionsunterricht bekannt, mit Adam, Nuh, Ibrahim, Ismail, Yunus, Musa und Isa ${ }^{9}$ (Friede sei mit ihnen allen), Vorbildern gelebten Glaubens in ihrer Zeit und für unsere Zeit.

Ein solches orientierendes Grundwissen stärkt den Glauben an den einen und einigen Gott, der sich über Gibril ${ }^{10}$ Muhammad (s.a.s.) in Liebe geoffenbart hat, der Hoffnung auf Rechtleitung im Dunya ${ }^{11}$ und Rahme ${ }^{12}$ im Achira ${ }^{13}$ schenkt.

\footnotetext{
9 Die Übersetzung der islamischen Prophetennamen: „Nuh“ = Noah; „Ibrahim“ = Abraham; „Ismail“" ist für die Muslime der ältere Sohn Abrahams, der geopfert werden sollte; „Yunus“= Jonas; „Musa“ = Moses; „Isa“ = Jesus.

10 „Gibril“ ist der Engel der den Propheten die göttliche Offenbarung brachte.

11 Arabisch: Diesseits
} 
Schließlich zeigt der Religionsunterricht auch die Weite der in allen Kulturen verwurzelten Ummah ${ }^{14}$ und weckt die Bereitschaft, Verantwortung zu übernehmen und sich zu engagieren. Religiöses Grundwissen ist wichtig für alle Kinder und Jugendlichen von der Grundschule bis zum Gymnasium und zur Berufsschule. Das gilt für die jungen Leute, die in einem muslimischen Elternhaus aufwachsen ebenso wie für diejenigen, die religiös distanziert leben und die eigene Erfahrungen mit dem Islam oder überhaupt einem Glauben aufbauen möchten.

Der konfessionell geprägte schulische Religionsunterricht bietet den muslimischen Schülerinnen und Schülern die einzigartige Chance, den Islam von innen heraus zu verstehen, und von außen zu betrachten. Somit trägt er dazu bei, den eigenen Standpunkt zu reflektieren und vernünftige Rechenschaft über den Glauben zu geben. In einer Zeit, in der Werte nicht mehr allgemein gültig sind, vermittelt der islamische Religionsunterricht eine Orientierung in ethischen Fragen, die in Qur'an und Sunnah gründen. Wer das Doppelgebot der Gottes- und Nächstenliebe (as-salah wa az-zakah ${ }^{15}$ ) kennt, wird auch sein Handeln daran ausrichten können.

Die öffentliche Schule, in der alle Schulpflichtigen zusammenkommen und verschiedene soziale und kulturelle Milieus und sprachliche und ethnische Herkünfte sowie verschiedene Ausprägungen des Islam vertreten sind, ist ein Spiegelbild unserer Gesellschaft, die religiös und weltanschaulich pluraler wird. Kinder und Jugendliche lernen sich über Konfessions- und Religionsgrenzen hinaus kennen und schließen nicht selten auch Freundschaften. Dieses Miteinander im Schulalltag bildet eine gute Möglichkeit, sich selbst und die anderen kennen zu lernen. Die muslimischen Kinder lernen, dass die Einheit des Islam und der Ummah höchstes Gut, dass Unterschiede innerhalb des Islam ein Reichtum und nicht dazu bestimmt sind, einander wertend oder gar ausgrenzend zu unterscheiden.

Aus unserer Sicht ist das glaubwürdige Zeugnis der Religionslehrerinnen und -lehrer für einen erfolgreichen Religionsunterricht unabdingbar und von entscheidender Bedeutung. „Möge das von gegenseitiger Liebe geprägte Vorbild des Gesandten Allahs (s.a.s.) das religiöse Lernen in der Schule prägen und dem Religionsunterricht seine ethische und spirituelle Mitte geben! Das wünsche ich allen Lehrenden in den Schulen und unserer jungen Generation auf ihrem Weg zum Frieden mit Allah und den Menschen. In dieser Linie greife auch ich aus meiner Verantwortung als Vorsitzender des Landesverbandes der Muslime in Niedersachsen das Thema erneut auf, um alle zu ermutigen und zu bestärken, die im Feld der religiösen Erziehung und Bildung Verantwortung tragen. Ich freue mich, dass es uns in Kooperation mit dem Pädagogischen Seminar der Georg-AugustUniversität gelungen ist, diese Tagung zu organisieren. Unser besonderer Dank

12 Arabisch: Barmherzigkeit

13 Arabisch: Jenseits

14 Arabisch: Gemeinschaft der Muslime

15 Das islamische Gebet und die islamische Armensteuer, bzw. Armenabgabe 
gilt Frau Dr. Katja Koch, Herrn Kinan Darwisch sowie Herrn Firouz Vladi, ohne deren Mitarbeit diese Tagung nicht zu standen gekommen wäre.

Wassalam, Avni Altiner,

Vorsitzender der Schura Niedersachsen

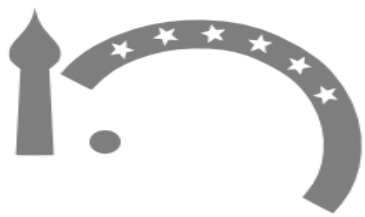


Ein historischer Blick auf den Islam 


\section{Gerdien Jonker: Den Islam erzählen. Interpretationen der muslimisch geprägten Welt in europäischen Schulbüchern}

Der Islam hat einen spezifischen Platz im europäischen Gedächtnis. Seine Wahrnehmung ist allerdings eine konfliktgeladene, die vor allem die bewaffneten Auseinandersetzungen aufzählt. Der Islam, ob nun in Gestalt von Arabern, Osmanen, Tartaren oder Türken, wird darin ,als der gefährlichste und dauerhafteste Feind Europas empfunden, als Europas Antithese und Negation" (Francois/Schulze 1999: 25).

Nicht nur die beiden Historiker Francois und Schulze kamen zu dieser Schlussfolgerung. Eine lange Reihe Wissenschaftler, die sich im Laufe des 20. Jahrhunderts mit dem nunmehr tausendjährigen Corpus europäischer Quellentexte über Muslime, darin abwechselnd „Sarazenen“, „Hagarener“, „Heiden“, „Musulmaner“, „Mooren“ oder „Türggs“ genannt, auseinandersetzte, stellte fest, dass die muslimischen Bevölkerungen an den Rändern Europas stets den Katalysator für die europäische Selbstwahrnehmung bildeten. Muslime galten als ,nicht wir' und wurden als die ,Anderen' schlechthin dargestellt, als der Feind vor den Toren Europas. Die Soziologie bemerkt dazu kühl, ohne Abgrenzung sei eine kollektive Identität nun einmal nicht zu haben. In diesem, für jede Gruppenbildung also 
unabdingbaren Abgrenzungsprozess galten Muslime somit als eine ,nicht wir'Gruppe. Die Grenzziehung zwischen der als ,eigen’ verstandenen Gruppe und den Fremden verlief allerdings nicht nur zum Süden hin - zum muslimischen Spanien, zur europäischen Türkei und zum anderen Ufer des Mittelmeers -, sie verlief ebenfalls quer durch die europäischen Städte und Landstriche. Hier verfolgte sie das Ziel, die jüdischen Gemeinden als nicht zugehörig darzustellen. Beide Wahrnehmungen und die damit verbundenen Ausgrenzungen komplettierten einander. Sie haben - jede auf ihre eigene Art - dazu beigetragen, Formulierungen dafür zu finden, was Europa ist, wo es liegt und welches seine Werte sind.

Dieser Beitrag handelt vom engen Verhältnis zwischen den Fremdbildern, die Muslimen einen bestimmten, außerhalb von Europa liegenden Ort zuwiesen, und den Selbstbildern Europas, die durch jene Fremdbilder ihre Konturen erst erhielten. Als Quellenmaterial wurden unter anderem die Narrative über die Muslime und den Islam in europäischen Geschichtsschulbüchern herangezogen. Geschichtsschulbücher enthalten Vorstellungen darüber, woher wir kommen und wohin wir gehen, wer wir sind und wie wir uns von anderen unterscheiden. Sie repräsentieren das, was gesellschaftlich an die nächste Generation weitergegeben wird, und vermitteln somit einen Kernbestand von Wissen, Weltbildern und Regeln.

Historisch gesehen wurden Europas Bewohner erst durch den nationalen Geschichtsunterricht zu Bürgern (citizens), die eine gemeinsame Sprache sprechen, dasselbe Territorium bewohnen und eine Geschichte teilen. Auch heute noch entstehen Bürger im Medium der (Geschichts-)Schulbücher, in denen nationale Narrative verdichtet und vermittelt werden. Daher lassen sich Schulbücher auch wie Klassifikationsschemata betrachten, wie kognitive Karten des Verstehens. Für das Studium von Selbst- und Fremdbildern bieten sie eine unverzichtbare Grundlage.

Bevor wir einen Rundgang durch die verschiedenen Quellenlagen machen, noch ein Wort zu den Eigenschaften von Selbst- und Fremdbildern. Das Wichtigste ist wohl: Selbst- und Fremdbilder sind kollektive Bilder. Kollektive Bilder sind wiederum Vorstellungen, die mit Hilfe spezifischer Sprachwendungen immer wieder in Erinnerung gerufen und damit gleichsam „gemacht“ werden. Sie fassen das Wesentliche zusammen, ohne sich um Zwischentöne oder gar Abweichungen zu kümmern, und werden bei Bedarf in jeder Lage wiederholt. Indem ,wir'Gruppen ,Anderen' stets zuschreiben, was sie nicht sind (sein wollen), bestätigt ihre Fremdbeschreibung fortlaufend ihre Selbstbeschreibung. Man könnte sagen, dass ein Fremdbild nichts anders als eine Funktion des Selbstbildes ist. Erkenntnisse über die andere Gruppe bringen sie in nur sehr geringem Maße.

Selbstbeschreibungen sozialer Gruppen sind per Definition normativ and damit auch konstitutiv. Die Gruppe legt fest, wie sie sein soll, und stellt damit die Weichen für die entsprechenden Sozialstrukturen, die die Umsetzung ihrer Vorstellungen ermöglichen sollen. Systemtheoretiker erklären daher, dass die Grenze 
zum ,nicht wir' durch die Sozialstrukturen gezogen werde. Wenn sich die ,wir'Gruppe zum Beispiel als demokratisch und modern beschreibt, als individuell und den Menschenrechten verpflichtet, dann müssen ihre Sozialstrukturen garantieren, dass dem auch so ist. Ihre Gegenspieler, die so genannten ,Anderen', bekommen inzwischen die Etikette, die das, Wir' partout nicht haben will: mittelalterlich und autoritär, rechtlos, gewalttätig und in einer instrukturellen Ungleichheit verhaftet. Akzeptieren diese ,Anderen' das ihnen Zugeschriebene nicht, so entstehen Spannungen. Man spricht von contested stereotypes, die wiederum drei Eigenschaften zu haben scheinen: sie entstehen in bestimmten historischen Situationen, sie sind langlebig und sie weisen eine ausgeprägte Dynamik auf. In den meisten Fällen sind beide Parteien, die ,wir'-Gruppe und die als anders Etikettierten, eng miteinander verflochten, zum Beispiel die europäische Kultur: der Aufklärung verpflichtet und der Orient, dem Europa im Laufe der Zeit ein wahres Potpourri an negativen Eigenschaften in die Schuhe geschoben hat: barbarisch und exotisch, rückständig und fremdartig, nicht vertrauenswürdig und mit einem generischen Zug zur Gewalt ausgestattet.

In gebotener Kürze sollen im Folgenden die europäischen Erzählmuster über den Islam in drei Schritten skizziert werden. Der erste Schritt stellt das tausendjährige Wissen über Muslime in Europa vor: was wusste man zu welcher Zeit und was wurde mit diesem Wissen gemacht? Der zweite bietet einen Einblick in das Kaleidoskop europäischer Schulbucherzählungen über den Islam: Was wird wo erzählt? Der dritte verortet das Islam-Narrativ der gegenwärtigen deutschen Geschichtsschulbücher in diesen beiden Kontexten.

Das Erkenntnisinteresse des Beitrags liegt darin, in Erfahrung zu bringen, inwiefern, mit welchen Mitteln und zu welchen Zwecken Europäer seit nunmehr tausend Jahren ihre muslimischen Nachbarn als generisch anders, oftmals auch als feindlich definieren. Die Leitfrage lautet: Ist das historische Erbe heute noch relevant für Repräsentationen der Zugehörigkeit zu Europa?

\section{Wissen über Muslime in Europa}

Gab es eigentlich in Europa vor den Kreuzzügen Wissen über Muslime, und wenn ja, welches? Die Crónica mozarabe von 754 berichtet zwar über den Widerstand der Karolinger bei Tours und Poitiers gegen die arabischen Streifzüge, aber dem Historiker Michael Borgolte zufolge war ,Poitiers' keineswegs die Entscheidungsschlacht zwischen Christen und Muslimen, zu der sie im 19. Jahrhundert stilisiert wurde. Die arabischen Streifzüge fielen zwischen den vielen Einwanderern und Plünderern an Europas Grenzen kaum ins Gewicht (vergl. Borgolte 2006: 259). „Nein“, folgert auch Almut Höfert in ihrem grundlegenden Werk über die „Türkengefahr" (Höfert 2003). Erst mit den militärischen Raubzügen Richtung Jerusalem entstand ein kriegerisches Epos, was verdecken sollte, dass die Franken in den Augen der Muslime als rohe Zeitgenossen galten und dass die Konfrontation mit 
der orientalischen Kultur auf europäischer Seite Unterlegenheitsgefühle ausgelöst hatte (vergl. Fontana 1995; Cardini 2000).

Europas ,Andere' waren zunächst die kultivierten und gelehrten Araber in Spanien und Süditalien, die die Lateiner zwar philosophi nannten, denen sie aber dennoch den Besitz einer Religion eifersüchtig absprachen. Spätestens seit die Türken vor Wien standen, begann Europa, „den Feind zu beschreiben“ (Höfert 2003) und sich zudem in der Retrospektive als christlich zu definieren (vergl. Borgolte 2006:300). Infolge dessen bekam auch der ,Feind' einen religiösen Anstrich, was sich alsbald zu einer Essentialisierung ausweitete. Der französische Historiker August Braudel schrieb dieses Spezifikum der europäischen Wahrnehmung auch den muslimischen Nachbarn zu: „Die islamische Welt verhält sich zum Okzident wie die Katze zum Hund. Es ließe sich auch von einem Gegen-Okzident reden, wobei die Zweideutigkeit jenes tiefen Gegensatzes, der zugleich Gegnerschaft, Feindschaft und Anleihe bedeutet, mitschwingen. Germaine Tillion würde von ,komplementären Feinden' reden. Sie sind tatsächlich gewaltige Feinde und Rivalen. Was der eine tut, tut auch der andere.“ (1958).

In der Konfrontation mit Muslimen in Palästina, auf Sizilien und in Spanien blitzt zum ersten Mal der Gegensatz christlich - nicht-christlich als bedeutungsvolles Moment der Selbstvergewisserung auf. Es geht zunächst mit der Vorstellung vom „Abendland“ als Bezugspunkt für kollektive Selbstbeschreibungen einher. Diese Verschiebungen vollziehen sich langsam und unstetig. Das Wort Europa als Selbstbezeichnung kommt erst in einem späteren Stadium hinzu (vergl. Burke 1980). Wann aber erhält der Begriff Europa eine neue Stellung? Dafür sind die folgenden Konstellationen relevant:

- Gegenüber der Türkengefahr treten Unterschiede zwischen Ost- und Westkirche zurück, und Europa wird als christliches Territorium beschrieben.

- Mit der Eroberung neuer Weltregionen erfolgt die Verschiebung des Horizonts - weg von Südost- und Osteuropa mit seinen orthodoxen und muslimischen Bevölkerungen hin zu Westeuropa.

- Die konfessionellen Spaltungen des 16. und 17. Jahrhunderts stellen einen tiefen Einschnitt und eine Zerrreißprobe dar. In dieser Umbruchzeit entsteht Europa als christliche Selbstvergewisserung und in Abgrenzung vom Islam.

Die Türkengefahr setzt dabei neben einer äußeren auch eine innere Abgrenzung in Bewegung. In den Reiseberichten und Wissenskompendien des Mittelalters wurden die Muslime bereits als leibhaftige Teufel und der Koran als ein Blendwerk voll von Lügen dargestellt. Als aber ab Mitte des 15. Jahrhunderts die lateinische Christenheit sich mit militärisch überlegenen Osmanen konfrontiert sah, bildete das überlieferte Wissen fortan die Folie, auf der empirische Berichte und damit neues Wissen über die Osmanen präsentiert wurden. Im Zeichen der Medienrevolution um 1500, die neue Vervielfältigungsmöglichkeiten und die rasante Verbreitung des medial propagierten Türkenbildes ermöglichte, verdichtete sich dieses zu einem Antagonismusnarrativ (vergl. Höfert 2003), einer Erzählung also, die 
gerade zum Ziel hat, der Antithese und der Negation der als anders erfahrenen ,Anderen' Ausdruck zu verleihen.

Das erste vollständige Produkt der Druckerpresse im Oktober 1454 war nicht etwa die Bibel, sondern ein Turcicum, ein so genannter Türkenkalender, das früheste gesicherte Druckwerk, das zur Propagierung eines Kriegs gegen die Türken diente: Eyn manung der cristenheit widder die durken. Ihm folgte im Dezember des gleichen Jahres der Türkenkalender für 1455 (vergl. Höfert 2003:58). Anschaulich wird hieran auch die von Benedict Anderson vertretene These der Allianz von Buchdruck und Reformation, also die enge Koppelung von konfessioneller Spaltung und Medienrevolution (1991). Diese Grenzziehungen sind gleichwohl nicht eindeutig. Auf dem Frontispiz vom „Theater wichtiger Städte der Welt“ von Georg Braun (1594), auf dem weibliche Gestalten die Kontinente und prächtig gekleidete Männer die zivilen Bürger der Zeit verkörpern, wird zum Beispiel ein Osmane in einem intensiven Gespräch mit seinen beiden Nachbarn dargestellt ( vergl. Schneider 2006:13). Schneiders Rundgang durch die Enzyklopädien der frühen Neuzeit macht klar, dass die Osmanen zugleich der zivilisierten Welt zugerechnet wurden.

Im Übergang zum 18. Jahrhundert findet der bewundernde Blick - mit abschätzigen Meinungen vermischt - Eingang in Darstellungen, die die Osmanen als exotisch und effeminiert hochstilisieren. In diesem Jahrhundert der Aufklärung und Zivilisation, der Rationalität und der Wissenschaft setzt sich die gelehrte Welt auch mit dem Koran auseinander, insbesondere durch die Übersetzungstätigkeit. Im Urteil der Übersetzer, das stets im Vorwort kundgetan wird, dominiert zwar über den Islam das Wort Lügengespinst, es setzt sich aber zugleich auch der Versuch durch, sich dem Werk objektiv, also wissenschaftlich zu nähern (vergl. Rehrmann 2001). In Deutschland wird hiermit die Basis für die philologische Arbeit mit dem Corpus der islamischen Tradition gelegt. In Frankreich und den Niederlanden vermischt sich die Beschäftigung mit den Erfahrungen (empirisches Wissen!) der Kolonialverwaltungen.

Es bleibt gegenwärtig schwierig einzuschätzen und stellt ein Desiderat dar, wie diese unterschiedlichen Überlieferungsstränge der Literaten, Philologen und Philosophen im 19. Jahrhundert ins Wissen Eingang fanden. Das reich bebilderte Buch Mythen der Nationen illustriert jedoch, dass der Islam im Zuge der Nationalstaatenbildung bereits einen spezifischen Platz in der Populärkultur vieler europäischer Länder zugewiesen bekommen hatte (vergl. Flacke 1998). Der Charakteristik nach handelte es sich bei diesen Darstellungen um die bereits eingangs erwähnte konfliktgeladene Wahrnehmung, in der sich vor allem die bewaffneten Auseinandersetzungen aneinander reihten. Der Islam - gemeint waren die Bevölkerungen, die mehrheitlich muslimisch waren - wurde darin als Feind Europas empfunden. Damit war Europas alter ego - was es selbst nicht sein wollte - fest in der Selbstwahrnehmung verankert. Den Wissenschaftlern blieb somit nur noch festzustellen, dass die muslimischen Bevölkerungen an Europas Grenzen einen Katalysator 
für die Selbstwahrnehmung bildeten. Insbesondere die südosteuropäische Grenze hielt die ungesicherte Identität Europas wach (vergl. Todorova 1997), aber auch die Selbstwahrnehmung der christlichen Spanier, die das muslimische Spanien bis heute größtenteils aus dem kollektiven Gedächtnis bannen (vergl. Viladrich-Grau, Mercè 2009) sowie die zahllosen Versuche des russischen Zentrum die Alleinherrschaft einer orthodoxen Identität zu installieren und jede Erinnerung an seine umfangreichen autochthonen muslimischen Bevölkerungen auszumerzen (vergl. Gibatdinov 2009).

Schließlich sei angemerkt, dass die zweite Medienrevolution am Ende des 20. Jahrhunderts die alte Wahrnehmung mit einer neuen Bilderflut überlagerte, deren Niederschlag auch in den heutigen Geschichtsschulbüchern zu finden ist (siehe unten). In der Forschung wird daher die Frage verhandelt, ob das Muster des früheren „Antagonismus-Narrativs“ die Kriterien für die Auswahl neuer Bilder über Muslime (Fundamentalismus, Terror) weiterhin anleitet (vergl. Hafez/Richter 2007).

Bietet das ,alte' europäische Wissen über den Islam Sedimente, die durch gegenwärtige Herausforderungen (Stichwort: 11. September 2001) und demographische Veränderungen (Migration) lediglich aktualisiert werden? Oder werden sie durch EU-Normen allmählich überlagert? ,Passt' das historische Erbe noch?

\section{Das Kaleidoskop der europäischen Schulbuch- erzählungen}

In den europäischen Schulbüchern folgt das Thema „Islam“ den Fußspuren dieser althergebrachten Wahrnehmung (siehe für den folgenden Abschnitt auch Jonker 2008). Es wiederholt Erzählfetzen kollektiver Erinnerungen und historischer Phantasien, die zwar praktisch überall in Europa negativ geprägt waren, aber dennoch in Süd- oder Nord-, Ost-, Zentral- oder Westeuropa mit jeweils anderen historischen Eindrücken angefüllt worden sind. Die arabische Expedition, die 732 bei Poitiers und Tours scheiterte, wurde 1851 von Edward S. Creasy als einer der 15 ,entscheidenden Schlachten der Welt“ gekürt, als „Europas Rettung vor dem zivilen und religiösen Joch des Koran“ (Creasy 1994: 157). Seitdem findet sie Aufnahme in den deutschen Schulbüchern, nicht aber in französischen oder spanischen. Der Mythos der Kreuzzüge ist seit dem 19. Jahrhundert ein Dauerbrenner in polnischen, österreichischen, deutschen und niederländischen Geschichtsbüchern, nicht aber in den skandinavischen Ländern oder in Italien. Russische, baltische und polnische Geschichtsbücher assoziieren „Islam“ zuallererst mit „Tataren“, jener mittelalterlichen Bezeichnung der Gruppe von Mongolen, die bis ins westliche Polen vorgedrungen waren (tartarus $=$ Hölle). Niederländer, Engländer und Franzosen wissen nicht um diese europäische Wahrnehmung, sondern betrachten die islamische Welt durch die Linse ihrer Kolonialvergangenheit. 
Italiener und Spanier, aber auch Norweger und Schweden verbinden mit „Islam“ den freien Handelaustausch. Die ersten beiden betrachten heute nur zögernd die Orte und Zeiten, in denen sie selber zur pax arabica gehörten, als Teil der eigenen Geschichte. Schon das allein ist wiederum für die Griechen ein Ding der Unmöglichkeit. Dementsprechend gehört die vierhundertjährige Periode der osmanischen Herrschaft im griechischen Schulbuch nicht zur eigenen Geschichte und verschwindet in äußerst knapp gehaltenen ,Feind'-Beschreibungen. Island ist das einzige europäische Land, das bisher kein Islam-Narrativ in seinen Schulbüchern anbietet. Der Grund dafür dürfte sein, dass die Insel nicht nur keine historischen Erfahrungen mit fernen, andersgläubigen Nachbarn hatte, sondern dass die Isländer in ihrer Geschichte auch kein Interesse an einer Abgrenzung verfolgten.

Diesen verschiedenen Narrativen über den Islam bilden kleine Erzählblöcke im Gesamtnarrativ der nationalen Geschichtsschulbücher. Der Lehrstoff für die sechste Klasse beginnt in der Regel mit der Steinzeit („Wo kommen wir her?“), um sich dann der „Wiege der Zivilisation“ zuzuwenden: dem alten Griechenland und dem römischen Reich, der Zeit der Völkerwanderung mit der Ankunft der Germanen in Europa und deren Verortung in einer christlichen Gemeinschaft. Ist dieser Bogen gespannt, dann haben die arabischen Stämme ihren Auftritt als „Eroberer" an den Küsten Europas oder als eine der „drei Weltreligionen“. Das begleitende Bildmaterial zeigt meistens die Kaaba, Massenansammlungen von betenden Gläubigen, berühmte Moscheen und verschleierte Frauen.

Auf dieser historischen Grundlage, die sich örtlich vom 17. Jahrhundert bis in die Gegenwart nachvollziehen lässt (siehe unten), halten seit dem 11. September 2001 neue Erzählungen Einzug in die Schulmedien. Islamismus, Terrorismus, rauchende Türme, schwarz verhüllte Frauen, abgehackte Hände und Steinigungen werden als Sinnbilder des Islam dargestellt. Die alte Essentialisierung des „Anderen“ als religiös, rückständig und nicht kompatibel mit europäischen und „westlichen" Werten erfuhr damit einen neuen Aufschwung.

Zusammenfassend kann erstens festgehalten werden, dass die unterschiedlichen Regionen Europas bis heute ihre Klischees und Stereotype über die muslimischen Nachbarn weitervermitteln, ohne sich über deren Herkunft und Sprengkraft groß Gedanken zu machen. In Österreich hat seit 2003 die Erzählung Hilfe, die Türken kommen! Eingang gefunden. In den polnischen Geschichtsbüchern schauen neben Osama bin Laden die schwarz verhüllten Frauen von den Seiten hoch. In Russland rösten die Tataren nach wie vor die armen Russenkinderchen am Spieß. In England stellt man Muslime gleich mit dem Mittelalter. Spanien wartet mit Karten der Vertreibung auf, und die Niederländer - und nicht nur sie diskutieren „van Gogh“ - Theo van Gogh, den 2004 ermordeten „Islamkritiker“.

Es scheint so, als seien die europäischen Schulbuch-Erzählungen über den Islam Facetten eines europäischen Gründungsnarrativs, dessen Elemente und aktuelle Anreicherungen in einer longue durée verankert sind. In ihr haben verschiedene lokale historische Erfahrungen ihre Spuren hinterlassen. Ihre vorläufige Kar- 
tografie in den verschiedenen Teilen Europas zeigt uns ein kaleidoskopisches Bild. Südliche Länder (Spanien, Italien) integrieren die Begegnungen im Mittelmeerraum und die Reconquista. Die zentraleuropäischen Länder der ehemaligen Österreichisch-Ungarischen Monarchie halten die Erinnerung an die Türkengefahr wach. Die östlichen Länder Russlands bringen gegenwärtig (wieder) alte Bilder von blutrünstigen Tataren zur Darstellung. Die westlichen Länder (England, Frankreich, Niederlande) vermischen koloniale Erfahrungen mit der Realität der Migration.

\section{Muslime und Islam in den deutschen Geschichts- schulbüchern}

Das gegenwärtige deutsche Narrativ fußt auf einem Erzählmuster, das in den ersten deutschen Geschichtsschulbüchern, die nach dem dreißigjährigen Krieg überall in Nord- und Mitteldeutschland erschienen, entwickelt worden war. Die flexible Fortschreibung dieser Texte scheint typisch für die protestantische Produktion von Geschichtsbüchern in den deutschen Ländern zu sein. Außer Berlin haben wir Erfurt, Helmstedt, Gotha und Nürnberg als Produktionsorte von Geschichtsbuchgenealogien identifizieren können (siehe für den folgenden Abschnitt auch Jonker 2008).

Geschichte war bis dahin eine Art Rumpelkammer gewesen, die dazu gedacht war, das Fach Latein mit Faktenwissen zu unterstützen (Fuhrmann 1999: 126). Die vielen Auflagen und die weite Verbreitung von Geschichtskompendien a studioso iuventute aus der Hand spätantiker Autoren zeugen indes davon, dass die Annäherung an die Geschichte später in den Schulen in Gebrauch blieb. Daneben entstanden in lutherischen Städten wie Gotha, Erfurt, Jena, Nürnberg, Helmstedt und Berlin aber auch Cluster von Gelehrten, die unter der Schirmherrschaft eines Fürsten ein neues Geschichtsbuch für „ihre“ Schule schrieben. Mit dabei waren meistens der Rektor des Gymnasiums, der örtliche Pfarrer, ein Theologe, ein Spezialist für antike Sprachen und ein Verleger; die Co-Autoren fanden meist in der Widmung eine Erwähnung, so zum Beispiel im frühesten Geschichtswerk lutherischer Provenienz, der Historicae Geographicae von Sebastian Schröter, das bereits 1614 in Erfurt verlegt wurde. In diesen Büchern fand nicht nur die Faktenanhäufung über Kreuzzüge, Sarazenen und Türkenkriege aus dem evangelischen Geschichtskalender einen Platz, sondern es wurden auch wiederholt Versuche unternommen, das gesamte historische Wissen übersichtlich und sinnvoll zu ordnen. So entstanden Geschichts- und Geographiekompendien, die über mehrere Generationen immer wieder von neuem geordnet und fortgeschrieben wurden, bis sich ein ,Master-Narrativ' abzeichnete, das den Vorstellungen der lutherischen Fürsten und Experten entsprach. Wie das im Einzelnen ablief, soll im Folgenden am Bei- 
spiel der Geschichtsbücher des Joachimthalschen Gymnasiums in Berlin erklärt werden.

1682 publizierte der Rektor des Joachimthalschen Gymnasiums in Berlin eine kleine Abhandlung, in der er die Geschichte der gesamten Welt ins Auge fasst (vergl. Wilhelmi 1682). Offensichtlich hatte das Büchlein seine Leserschaft auch über das Gymnasium hinaus erlangt, jedenfalls ergriff der Verleger einige Zeit später die Initiative, die ursprüngliche Ausgabe von 154 Seiten auf nunmehr 1.070 Seiten auszudehnen (vergl. Wilhelmi 1696). Der Text wurde inhaltlich in fünf Weltreiche unterteilt, das Resultat erscheint aus heutiger Sicht dennoch als unübersichtliche Anhäufung biblischen, antiken und kirchengeschichtlichen Wissens. Hinzu kam ein Sammelsurium an Kalendereintragungen aus neuerer Zeit, von den Kreuzzügen über osmanische Niederlagen im Felde bis hin zur Sichtung eines Kometenschweifs in Form eines türkischen Säbels. Es waren allesamt kurze, zusammenhangslose Eintragungen nach dem folgenden Erzählmuster: „Unter Pabst Urbano Anno 1095 haben die Christen das heilige Land wieder erobert/ und es 88 Jahr einbehalte. In dieser Zeit ist der Ordo Canonicorum seu Regularium aufkommen Anno 1070/ wie auch die Karteuser von Brunone." (Wilhelmi 1696: Monarchiae Quartae/Imperatores Christiana)

Die zweite Ausgabe war aber offensichtlich kein Erfolg; sie wurde zumindest nicht wieder aufgelegt. Dafür erschien bereits 1723 aus der Hand von Hilmar Curas, Lehrer am selbigen Gymnasium, eine Einleitung zur Universalhistorie, die die Arbeit seines Vorgängers wieder aufnahm. Statt der Einteilung in Weltreiche, wählte der Autor diesmal eine teleologische, heilsgeschichtliche Strukturierung (Curas 1723). Curas teilte die Vergangenheit in drei übersichtliche Epochen, welche der christlichen Heilsgeschichte Zentralität einräumten, nämlich: „Von der Erschaffung der Welt biß auf die Sündfluth“; „Von der Sündfluth biß auf die Geburt Christi“, sowie „Von Christi Geburt biß auf diese Zeit“. Den jeweils anfallenden Stoff teilte er in Fragen und Antworten. Auf diese Weise kam er auch auf die Türken zu sprechen: „Was ist vom Constantino Paleologo zu merken? Er ist der letzte christliche Kayser gewesen, und zu seiner Zeit haben die Türken Constantinopel erobert. / Wie gieng es dem Kayser bey der Übergabe? Er kam in der Übergabe um das Leben, sein Haupt wurde auf eine Stange gesetzt und von den Türken herum getragen. Die Stadt wurde geplündert, alles niedergemachet, und grausam gehauset.“ (Curas 1723: Einleitung S. 88)

Diese kurze Eintragung schien aber doch auf ein Bedürfnis nach mehr Information zu stoßen. Jedenfalls fügte der Autor dem Buch noch einen achtseitigen Anhang hinzu, dem er den Titel Von den Türken gab. Hier findet sich der Beginn der Schulbucherzählung über „den Islam“. Curas`Fragen und Antworten produzieren ein Erzählschema, das das Islam-Narrativ in den deutschen Geschichtsschulbüchern bis heute bestimmt. Seine Frage „Wer war dieser Mohamet?" betraf das Leben des Propheten. Auf: „Wie befestigte er diese Religion?“ folgte eine Pauschalbeurteilung des Islam. Curas dritte Frage: „Wie fieng es weiter an?“ führte 
zur Geschichte der frühen Reiche. Mit: „Welche Länder bemächtigten sich die Saracener?" leitete er zum arabischen Angriff auf die europäischen Küsten im Mittelmeer über. Zahlreiche Fragen über Die türkische Bedrohung gaben ihm zudem Gelegenheit, die „Aggression“ der Angreifer im Detail darzulegen.

Ein halbes Jahrhundert später wurde das Werk erneut fortgeschrieben. Diesmal war es der Theologe und Historiker Matthias Schröckh, der sich daranmachte, die schlichten Fragen und Antworten seines Vorgängers in einem zweibändigen, 900 Seiten umfassenden Werk umzuschreiben, das später mit dem Titel Weltgeschichte für Kinder bis weit in das 19. Jahrhundert nachgedruckt werden sollte (Schröckh 1774). Die Erzählung Über den Türken wird darin unter dem Titel Über den Araber fortgeführt.

Ende des 18. Jahrhunderts war die akute Kriegsdrohung der Türken bereits in zeitlich weite Ferne gerückt; die lutherischen Bildungsschichten interessierten sich so für den Islam eher als eine „,natürliche Religion“ und für die Osmanen eher als Produzenten exotischer Kulturgüter. Türkische Alltagsszenen wurden in Meißener Porzellanfiguren abgebildet. Der preußische König schenkte dem türkischen Gesandten einen islamischen Friedhof in Berlin (vergl. Rehrmann 2001). Schröckh schrieb eine weitgehend säkularisierte Weltgeschichte. Darin stellte er den Islam im Kontext der Weltreligionen dar und betrachtete ihn unter rationalen, fast wissenschaftlichen Gesichtspunkten. Das Erzählschema blieb jedoch einfach gestrickt und orientierte sich im Wesentlichen an dem seines Vorgängers. Es umfasste die Schritte: (a) Mohammed steht für eine neue Religion; (b) dies führte zu Angriffen auf Europa; (c) dagegen mussten sich die Europäer mit Hilfe der Kreuzzügen verteidigen. Mit einer Erzählung von 50 Seiten nahm Schröckh übrigens auch jene ausgedehnten Erzählungen über „den Islam“ vorweg, die im 19. Jahrhundert die Geschichtsbücher dominieren sollten; Ludwig Bauer etwa widmete dem Thema insgesamt 160 Seiten (vergl. Bauer 1836).

Das gegenwärtige Narrativ führt Teile dieses historischen Erzählmusters fort. Seit den 1990er Jahren ist es um eine Erzählung über „Gastarbeiter“ aus der Türkei ergänzt worden, die von „Frauen“ und „Fundamentalismus“ flankiert wird. Demnach umfassen die gegenwärtigen Erzählelemente folgende Einheiten:

- Mohammed und die Entstehung des Islam

- Der Koran und die religiösen Pflichten

- Dschihad und arabische Kultur in Spanien

- Die Kreuzzüge, gefolgt vom „Leben im Heiligen Land“

- Die Begegnung der Religionen früher und heute

Gegen Ende der Gesamterzählung, wenn die Geschichtsbücher den Stoff für die 10. Klasse bereitstellen, folgt in manchen Werken ein Kapitel über „Reislamisierung und Fundamentalismus“. Andere wenden sich an dieser Stelle aktuellen Debatten zu, deren Themenpalette vom „Kopftuchstreit“ über „Moscheenbau in deutschen Städten“ bis zur Frage reicht, ob die Türkei Teil der Europäischen Union sein könne. Die neuesten Bücher zeigen Fotos von Osama bin Laden sowie 
die brennenden Twin-Towers nach den Anschlägen vom 11. September 2001 und diskutieren den clash of civilization.

\section{Zum Schluss}

Ohne Selbstbeschreibung kann sich weder kollektive Zugehörigkeit noch Integration artikulieren. Die Niederländer grenzen sich von den Deutschen ab und die Belgier wieder von den Niederländern. Die Briten ziehen eine Grenze zwischen sich und dem „Kontinent“, und dem neu erwachten russischen Patriotismus scheint es im Augenblick eine Notwendigkeit zu sein, sich von Westeuropa abzugrenzen. Die Amerikaner ziehen seit dem 11. September eine Grenze quer über den Globus, auf der einen Seite sehen sie ihre friends, auf der anderen Seite die rogue states. Europa grenzt sich von seinen muslimischen Nachbarn am anderen Ufer des Mittelmeers ab und zieht allerlei Grenzen quer über den Balkan, die seine Distanz zur Türkei zum Ausdruck bringen sollen. Manche dieser Grenzziehungen finden in diesem Augenblick statt, andere schauen auf eine lange Tradition zurück. Was sie gemein haben, ist dass sie in Zeiten von Spannung, der Empfindung von Bedrohung oder Kriegsgewalt entstanden sind. Kollektive Erinnerungen, die durch Angst hervorgerufen wurden, haben bekanntlich einen langen Schatten, länger als die längsten Zeiten friedlicher Koexistenz.

Dennoch verschieben sich solche Grenzziehungen ständig. Solange sie die gegenwärtige Selbstwahrnehmung unterstützen und für die ,wir'-Gruppe nützlich sind, können historische Sedimente mit Aktualität aufgeladen werden. Verlieren sie diese Rolle, so verschwinden auch die Feindbilder. Niederländer porträtieren den Deutschen gerne als Nazi-Schurken, der vor siebzig Jahren das Land besetzt hielt, aber wenn es um Geschäftspartner oder Ferienbegegnungen geht, lassen sie diese Zuschreibung schleunigst fallen. Großbritannien portraitiert sich selbst gerne als weltweites empire, das der EU finanziell haushoch überlegen ist, doch seit Beginn der Finanzkrise 2007/2008 überlegt es sich, dem Euroraum vielleicht doch beizutreten. Die muslimischen Nachbarn Europas haben ihre Spuren in den kollektiven Erinnerungen hinterlassen. Mit dem Ende des Ost-West-Konflikts, Anfang der 1990er Jahre, als man feststellte, dass im erweiterten Europa circa 22 Millionen Muslime leben, begannen EU-Politiker und Wissenschaftler, Nichtregierungsorganisationen und Nachbarschaftsinitiativen, Muslime und Nichtmuslime aktiv darüber nachzudenken, wie sich das Bild, das die einen vom anderen haben, in eine, wir'-Empfindung ummünzen lässt.

Sind aber die europäischen Erzählmuster über den Islam, die die Darstellungen über Muslime in Geschichtsschulbüchern beeinflussen, nun Ausdruck einer allgemein vorherrschenden Islamfeindlichkeit? Ich meine, dass sie vor allem Ausdruck althergebrachter Vorbehalte sind, die ihren Ursprung in längst vergessenen Ängsten haben. Dieses historische Erbe kann durch Aktualität durchaus neu entstehende Ängste unterfüttern. Die Aktualität kann sich aber auch dahingehend 
entwickeln, dass das Erbe seine Bedeutung verliert und verblasst. Entscheidend wird sein, wie sich Europa in den kommenden Jahren auf globaler Ebene definieren wird: als Draufgänger, als Kolonialmacht oder als eine unter vielen Weltregionen, die sich ihrer Abhängigkeit von anderen Teilen der Erde bewusst ist.

\section{Literatur}

Anderson, B.: Imagined Communities. Reflections on the Origin and Spread of Nationalism, 2. Aufl., London 1991

Bauer, L.: Allgemeine Weltgeschichte für alle Stände mit besonderer Rücksicht auf die Geschichte der Religionen sowie das Bedürfniß der gebildeten Jugend beiderlei Geschlechts, bearbeitet und bis auf das Jahr 1835 fortgeführt. Stuttgart 1836

Borgolte, M.: Christen, Juden, Muselmanen. Die Erben der Antike und der Aufstieg des Abendlandes 300 bis 1400 n. Chr. München 2006

Braudel, F.: „Histoire et sciences sociales”, in: Annales 14, S.710-18 1958

Burke, P.: „Did Europe exist before 1700?” in: History of European Ideas 1, S. 21-29 1980

Cardini, F.: Europa und der Islam. Geschichte eines Missverständnisses. München: 2000

Creasy, E. S.: Fifteen decisive battles of the World. From Marathon to Waterloo. New York: DaCapo Press, Nachdr. der Erstausgabe von 1851. 1994

Curas, H.: Einleitung zur Universalhistorie, Worinnen die merkwürdigste Begebenheiten von Anfang der Welt bis auf diese Zeit, in Fragen und Antwort kurz vorgetragen werden, nebst einem Anhang der türkischen Historie wie auch eine vollständige Genealogie der Churfürsten zu Brandenburg. Berlin 1723

Flacke, M. (Hrsg.): Mythen der Nationen. Ein europäisches Panorama. München u.a.1998

Francois, E./Schulze, H.: „Das emotionale Fundament der Nationen“, in: Monika Flacke (Hrsg.): Mythen der Nationen. Ein europäisches Panorama. Berlin 1999

Fontana, J.: Europa im Spiegel. Eine kritische Revision der europäischen Geschichte. München 1995

Fuhrmann, M.: Der europäische Bildungskanon. Frankfurt 1999

Gibatdinov, M. M.: „Cross-referencing Images of Muslims and Islam in Russian and Tatar Textbooks (1747-2007)", in: Gerdien Jonker/Shiraz Thobani (Hrsg.): Narrating Islam: The Muslim World in European Texts. London: 2009 
Hafez, K./Richter, C.: „Das Islambild von ARD und ZDF“, in: Aus Politik und Zeitgeschichte, 26-27, S. 40-46, 2007

Höfert, A.: Den Feind Beschreiben. Türkengefahr und europäisches Wissen über das Osmanische Reich 1450-1600. Frankfurt 2003

Jonker, G.: „Islam in German textbooks. The history of an educational narrative”, in: Luigi Cajani (Hrsg.): The Impact of Textbook on the Knowledge of Other Cultures. Madrid 2008

Jonker, G.: „Die longue durée der Islam-Erzählung: Genese und Wandel eines narrativen Schemas in deutschen Schulbüchern 1517-2001", in: Abdo Abboud/Ulrike Stehli-Werbeck (Hrsg.): Die Wahrnehmung des Anderen in der arabischen Welt und in Deutschland. Münster 2008a

Rehrmann, M.-O.: Ehrenthron oder Teufelsbrut? Das Bild des Islams in der deutschen Aufklärung. Zürich 2001

Schneider, U. J.: Seine Welt Wissen. Enzyklopädien der Frühen Neuzeit. Darmstadt 2006

Schröckh, M.: Einleitung zur Universalhistorie: zum Gebrauche bey dem ersten Unterrichte der Jugend; nebst einem Anhange der Saechsischen und Brandenburgischen Geschichte / Hilmar Curas ganz neu umgearb., berichtigt u. zum Gebrauch d. Schulen bequemer gemacht von Johann Matthias Schroeckh. Berlin 1774

Todorova, M.: Imagining the Balkans. Oxford 1997

Viladrich-Grau, M.: ,Representations of Muslim Andalus in the Scholarly Historical Texts of Catalonia (1714-1900)", in: Gerdien Jonker/Shiraz Thobani (Hrsg.): Narrating Islam: The Muslim World in European Texts. London 2009

Wilhelmi, J. G.: Universal-Historie in kleine Periodos oder Exercitia eingetheilet, umb der Jugend das studium historicum angenehme zu machen/. Berlin 1696

Wilhelmi, J. G.: Historia universalis, in welcher in kleinen Periodis oder Exercitiolis der Jugend das Studium historicum wird vorgebildet, zu dem Ende, daß sie zugleich mit der Composition auch die vornehmsten Geschichte und Ordnung der Zeiten erlernen möge. Berlin 1682 
Muslimische Kinder in der Schule 


\section{Katja Koch: Migrantenkinder im deutschen Schulwesen}

\section{Bildungserfolg und Kompetenzerwerb}

Der Anteil ausländischer Kinder und Jugendlicher im deutschen Schulwesen steigt seit einigen Jahrzehnten kontinuierlich an. Betrug ihre Zahl 1970 noch rund 55.000, besuchten im Schuljahr 2005/2006 bereits rund 930.000 ausländische Schülerinnen und Schüler allgemein bildende Schulen und rund 190.000 ausländische Schülerinnen und Schüler berufliche Schulen. Ihr Anteil an der Gesamtschülerschaft liegt damit in den allgemein bildenden Schulen bei knapp 10\%, in den beruflichen Schulen bei knapp 7\%.16 Gemessen an diesem Anteil, fällt ihre Bildungsbeteiligung jedoch eher gering aus. Ausländische Kinder und Jugendliche besuchen häufiger die Hauptschule und seltener das Gymnasium als deutsche Kinder und Jugendliche und sind im Vergleich zu ihren deutschen Altersgenossen insbesondere in der Sonderschule überrepräsentiert ${ }^{17}$. Zwar profitiert auch diese Gruppe von der im Kontext der Bildungsexpansion gestiegenen faktischen Bil-

16 Angaben des Statistischen Bundesamtes.

17 20\% aller ausländischen Schüler besuchen eine Hauptschule, jedoch nur 10\% der deutschen Schüler. Ein Gymnasium besuchen 10\% der ausländischen Schüler, jedoch 28\% der deutschen

Schüler. Eine Förderschule besuchen 7\% der ausländischen und 4\% der deutschen Schüler. 
dungsbeteiligung, der Abstand zu den gleichaltrigen deutschen Jugendlichen ist jedoch insbesondere im Bereich der höheren Abschlüsse noch beträchtlich. So ging z.B. im Zeitraum von 1984 bis 2003 der Anteil ausländischer Schülerinnen und Schüler, die ohne Hauptschulabschluss von der Schule abgehen, deutlich zurück, und gleichzeitig stieg der Anteil ausländischer Schülerinnen und Schüler, die die Schule mit einem Realschulabschluss oder mit der Hochschulreife verlieBen.

Betrachtet man die Bildungsbeteiligung nach Nationalitäten getrennt, so zeigt sich, dass einzelne Gruppen bildungserfolgreicher sind als andere. Obwohl sich die Eltern hinsichtlich des Bildungshintergrunds und der für ihre Kinder geäußerten Bildungsaspiration kaum unterscheiden, weisen griechische und spanische Kinder relativ hohe Besuchsquoten für Gymnasien auf, während italienische und türkische Kinder dort besonders selten anzutreffen sind ${ }^{18}$. Überproportional häufig sind italienische Kinder und Kinder aus dem ehemaligen Jugoslawien jedoch auf den Förderschulen vertreten. Die MARKUS-Studie identifizierte zudem Vietnamesen, Polen und Franzosen als eher bildungserfolgreiche und Albaner und Aussiedler als weniger bildungserfolgreiche Gruppen (vgl. Helmke u.a. 2002, S. 136). Auch bei der Einmündung in eine Berufsausbildung lassen sich diese ethnischen Unterschiede noch feststellen. Unter denjenigen Jugendlichen, die zwar einen Hauptschulabschluss erwerben, danach aber keine Ausbildung finden, sind insbesondere türkische und italienische Jugendliche überproportional häufig vertreten (vgl. Kristen 2003, S. 27).

Bisher lassen sich als Gründe für diese Unterschiede zwischen den Nationalitäten keine empirisch gesicherten Belege, sondern allenfalls Vermutungen anführen. Eine differenzierte empirische Untersuchung zu diesem Thema (vgl. Diehl 2005) kommt zu keinen eindeutigen Befunden und verweist darauf, dass intuitiv einleuchtende, aber kulturell vereinfachende Erklärungsmuster kaum Aussagekraft für komplexe wissenschaftliche und gesellschaftliche Probleme haben (vgl. auch Herwartz-Emden 2003, S. 690). Da Schulerfolg immer auch gekoppelt ist an die soziale Positionierung einer Familie sowie deren bildungsrelevante kulturelle Ressourcen und diese wiederum auch innerhalb einer ethnischen Gruppe deutlich variieren können, ist meiner Ansicht nach einfachen kausalen Beziehungen zwischen ethnischer Herkunft und Schulerfolg keine sonderlich große Relevanz beizumessen.

Betrachtet man nun die während der Schulzeit erreichten Kompetenzen genauer, dann lässt sich anhand der Ergebnisse der in jüngster Zeit veröffentlichten Leistungsstudien auch hier ein tendenziell schlechteres Abschneiden der Migrantenkinder feststellen. Gemeinsam ist den jeweiligen Studien, dass sie darauf zielen, Daten über die Lernstände der Schüler, aber auch über die Leistungsfähigkeit der Bildungssysteme zu generieren. Im Gegensatz zu den internationalen Leitungsstu-

18 16\% der spanischen und 11\% der griechischen Kinder besuchen ein Gymnasien, aber lediglich $6 \%$ der italienischen und $5 \%$ der türkischen. 
dien, die zumeist überblicksartig auf einen Vergleich unterschiedlicher (Bundes)Länder zielen (z.B. PISA, IGLU, DESI), geben regionale Leistungsstudien (z.B. MARKUS, KESS, LAU) Auskunft über die in einem Bundesland erreichten Lernstände. Mit den ausgewählten Bereichen sollen Basiskompetenzen erfasst werden, die notwendig sind, um am gesellschaftlichen Leben in persönlicher und wirtschaftlicher Hinsicht teilhaben zu können. Schlechte Ergebnisse in diesem Kontext implizieren daher für die Zukunft eines Individuums ungünstige Ausgangsbedingungen und geringere berufliche Chancen(vgl. z.B. Baumert/Artelt 2001, S. 8/9, Klieme/Beck 2006, S. 2/3). Für das Bildungssystem stellt es eine Herausforderung dar, die festgestellten Kompetenzrückstände durch gezielte Maßnahmen $\mathrm{zu}$ verringern.

Hinsichtlich des Kompetenzerwerbs von Migrantenkindern lassen sich bezogen auf die Ergebnisse der genannten Studien folgende Aussagen treffen:

1. Migrantenkinder erreichen im Vergleich zu den einheimischen Kindern niedrigere Kompetenzwerte.

Bei der Lesekompetenz überschreiten knapp 50\% der Jugendlichen aus zugewanderten Familien die elementare Kompetenzstufe I im Lesen nicht, und selbst wenn sich diese Kinder seit ihrer Geburt in Deutschland aufhalten, bleibt deren mittleres Leistungsniveau unter dem von Jugendlichen ohne Migrationshintergrund. Der Rückstand, den jugendliche Migranten in Mathematik gegenüber Jugendlichen ohne Migrationshintergrund aufweisen, beträgt in Deutschland ein bis zwei Schuljahre, wobei vor allem Kinder der ersten Generation (Eltern zugewandert, Kinder in Deutschland geboren) betroffen sind. So erreichen über 50\% der Jugendlichen, die in Deutschland geboren sind und deren Eltern aus der Türkei stammen, in Mathematik lediglich ein Kompetenzniveau, das nicht über die erste Kompetenzstufe hinausgeht (vgl. Ramm u.a. 2005, S. 281ff). Hinsichtlich der mathematischen Kompetenz verhält es sich etwas anders. Grundsätzlich liegt auch hier das Kompetenzniveau der Jugendlichen mit Migrationshintergrund unter dem der Jugendlichen ohne Migrationshintergrund, doch sind die Jugendlichen mit nur einem im Ausland geborenen Elternteil in Mathematik deutlich erfolgreicher als Jugendliche der ersten Generation oder Zugewanderte.

Von daher kann angenommen werden, dass sich Defizite in der Lesekompetenz kumulativ auf den Kompetenzerwerb in den Sachfächern auswirken (vgl. Baumert/Schümer 2001, S. 376f). Dies scheint jedoch vor allem ein Phänomen der Sekundarstufe zu sein. In der IGLU-Studie waren die Unterschiede zwischen Kindern mit und ohne Migrationshintergrund im Lesen und in den Naturwissenschaften ausgeprägter als in Mathematik. Offensichtlich hängen mathematische Kompetenzen in der Grundschule noch weniger von sprachlichen Voraussetzungen $\mathrm{ab}$ als in der Sekundarstufe. Insgesamt zeigen sich auch in IGLU Unterschiede zwischen Kindern mit und ohne Migrationshintergrund, die relativen Leistungsabstände der drei Gruppen (Kinder ohne Migrationshintergrund, Kinder mit 
einem im Ausland geborenen Elternteil und Kinder mit zwei im Ausland geborenen Elternteilen) sind dabei noch weitgehend gleich (je eine halbe bis eine drittel Standardabweichung). Die geringsten Leistungen erbringen aber auch hier die Kinder mit beiden im Ausland geborenen Eltern, die zu den „schwachen“ Schülern ${ }^{19}$ gehören (Schwippert/Bos/Lankes 2003, S. 285). In der Sekundarstufe hingegen nähern sich die Werte der Schülerinnen und Schüler ohne Migrationshintergrund und jener Schülerinnen und Schüler mit einem im Ausland geborenen Elternteil nahezu an, während die Leistungen der Schüler mit zwei im Ausland geborenen Eltern eine dreiviertel Standardabweichung unter diesen Werten liegen (vgl. Baumert/Schümer 2001, S. 377).

2. Eine Ausnahme bildet der Kompetenzerwerb in den Fremdsprachen.

Während Kinder mit Migrationshintergrund hinsichtlich ihrer Kompetenzen in Deutsch, Mathematik und Naturwissenschaften in den meisten Studien schlechter abschneiden als Kinder ohne Migrationshintergrund, lässt sich dieser Effekt bezogen auf die Fremdsprachen nicht erkennen. In den LAU-Studien 9-13 zeigen sich in Englisch lediglich geringe Leistungsrückstände zwischen ausländischen Kindern und Kindern ohne Migrationshintergrund (vgl. Lehmann u.a. 2002, S. 158, Lehmann u.a. 2004, S. 142, Lehmann u.a. 2005, S. 66). Die KESS-Studie zeigt, dass zwischen Kindern, deren Eltern beide in Deutschland geboren wurden, und Kindern mit einem im Ausland geborenen Elternteil keine Unterschiede im Hörverstehen Englisch bestehen ${ }^{20}$. Die Gruppe der Kinder, deren Eltern beide im Ausland geboren wurden, schneidet zwar auch im Vergleich mit diesen beiden Gruppen schlechter ab; Im Vergleich zu ihren Leistungen im Lesen, in Mathematik und Naturwissenschaften, in denen sie jeweils um die 90 Skalenpunkte erreichen, stellt das Hörverstehen Englisch mit 95 Skalenpunkten jedoch ihre relativ stärkste Kompetenz dar (vgl. May 2006). Die DESI-Studie schließlich bestätigt die positiven Effekte, die sich bei Kontrolle der Hintergrundvariablen (Schulform, sozioökonomischer Status) aus einem mehrsprachigen oder nicht-deutschsprachigen Hintergrund auf die Gesamtleistung in Englisch ergeben. Beim Erwerb einer weiteren Fremdsprache scheinen sich die beim Erlernen der Zweitsprache Deutsch von den Migrantenkindern angeeigneten metasprachlichen Kompetenzen als von Vorteil zu erweisen. Mehrsprachige Schülerinnen und Schüler und Schülerinnen und Schüler mit einer nicht-deutschen Herkunftssprache verfügen beim Erlernen einer Fremdsprache über ein Potenzial, „das sie von den Schülern deutscher Herkunftssprache deutlich unterscheidet“" (Klieme/Beck 2006, S. 26).

19 Zur Definition der Kompetenzen von „,schwachen“ Schülerinnen und Schüler vgl. Schwippert/Bos/Lankes 2003, S. 289

20 Beide Gruppen erreichen jeweils 105 Skalenpunkte, wobei in der KESS-Studie die Skalenwerte auf einem Mittelwert von 100 und einer Standardabweichung von 30 skaliert sind. 
3. Die Beherrschung der Unterrichtssprache ist ausschlaggebend für den schulischen Erfolg.

Trotz dieser Vorteile beim Erwerb der ersten Fremdsprache in der Schule zeichnet sich ab, dass vor allem die Beherrschung der Unterrichtssprache für den Schulerfolg ausschlaggebend ist. Die rheinland-pfälzische Studie MARKUS (vgl. Helmke u.a. 2002) kommt hier zu dem Ergebnis, dass insbesondere Schülerinnen und Schüler aus den traditionellen Anwerbestaaten, die zu Hause kein Deutsch sprechen, am schlechtesten abschneiden (vgl. Helmke u.a. 2002, S. 28). Während die PISA-Studie 2000 lediglich allgemeine Aussagen über den Zusammenhang von Sprachbeherrschung der Unterrichtssprache und Kompetenzerwerb macht (vgl. Baumert/Schümer 2001, S. 374), sind die diesbezüglichen Analysen der PISAStudie 2003 im Hinblick auf die sprachliche Akkulturation der Schüler differenzierter. Grundsätzlich zeigt sich die Tendenz, dass die durchschnittlichen Kompetenzen der jugendlichen Migranten im Lesen und in Mathematik einen umso gröBeren Abstand zu Jugendlichen ohne Migrationshintergrund aufweisen, je weniger sie die deutsche Sprache im Alltag verwenden. „Deutschsprachige“ Migranten erreichen deutlich höhere Kompetenzwerte als „fremdsprachige“ (vgl. Ramm 2003b, S. 284f). Dieser Befund deckt sich auch mit internationalen Forschungsergebnissen, wonach Jugendliche, deren Umgangssprache nicht die Unterrichtssprache ist, geringere Kompetenzwerte erreichen. Allerdings gilt dieser Zusammenhang nicht für alle Länder gleichermaßen: Während in Deutschland, Belgien, den Niederlanden und der Schweiz Schülerinnen und Schüler, deren sprachliches Medium nicht die Unterrichtssprache ist, besonders schlechte Leistungen erbringen, weichen die Leistungen derselben Gruppe von Jugendlichen in Neuseeland, Kanada und Australien nicht signifikant vom Durchschnitt der Gesamtstichprobe ab (vgl. Ramm u.a. 2004, S. 260).

\section{2. „Ursachenforschung“: Zur Diskussion um die Bil- dungsbenachteiligung von Migrantenkindern}

Als Ursachen für die im internationalen Kontext auffällige Benachteiligung von Migrantenkindern im deutschen Schulsystem werden - neben der Beherrschung der Unterrichtssprache - in der einschlägigen Forschung noch weitere Faktoren benannt:

Diefenbach/Nauck (1997) sowie Nauck/Diefenbach/Petri (1998) vermuten mit Bezug auf die Gesellschaftstheorie von Bourdieu, dass Bildungsbenachteiligungen von Migrantenkindern die Folge strategischer Handlungsentscheidungen ihrer Eltern im Hinblick auf den Einsatz und die Transferierbarkeit des kulturellen Kapitals in der Familie sind. Schulerfolg wird in diesem Modell als spezieller Profit verstanden, „den Kinder aus verschiedenen sozialen Klassen und Klassenfraktionen auf dem schulischen Markt erlangen können (...) d.h. schulischer Erfolg oder 
Misserfolg wird nicht (primär) als Folge natürlicher Fähigkeiten aufgefasst" (Diefenbach/Nauck 1997, S. 282). Ein geringes kulturelles und ökonomisches Kapital in der Familie macht das Erreichen höherer Bildungsabschlüsse eher unwahrscheinlich. Bisher erbringen die entsprechenden Untersuchungen für diese These keine eindeutigen Belege: Diefenbach merkt z.B. an, dass sich der geringe Bildungserfolg von Migrantenkindern nur teilweise durch Merkmale des familiären Hintergrunds der Kinder erklären lässt (vgl. Diefenbach 2005, S. 44). Dem widersprechen allerdings Kristen/Granato, die davon ausgehen, dass die Ausstattung der Familien mit bildungsrelevanten Ressourcen „von herausragender Bedeutung für den Bildungserfolg der Kinder“ seien (Kristen/Granato 2005, S. 141).

Eine besondere Bedeutung wird auch dem familiären Milieu und dem dort gepflegten Erziehungsstil beigemessen (vgl. z.B. Nauck/Özel 1986, Fuhrer/Mayer 2005, Uysal 1998). Uysal z.B. stellt fest, dass die Eltern türkischer Hauptschüler einen Erziehungsstil pflegen, dessen Zielsetzung mit Gehorsam, Unterordnung und Respekt vor den Eltern umschrieben werden kann. Sie erziehen ihre Kinder strenger und legen auf die Achtung der elterlichen Autorität großen Wert. Die Väter helfen seltener im Haushalt mit und orientieren sich an traditionellen Formen der Arbeitsteilung. Verstöße gegen traditionelle Verhaltensnormen sanktionieren sie häufiger mit Strafen, die durchaus auch körperliche Gewalt beinhalten können. Die Eltern türkischer Hauptschüler sind zudem stark religiös gebunden, was sich u.a. in einer Achtung der Regeln des Islams ausdrückt. Im Gegensatz hierzu scheinen die Eltern türkischer Gymnasiasten weltoffener, liberaler, toleranter und weniger an die Religion gebunden (vgl. Uysal 1998, S. 163ff). Im Hinblick auf die für das Kind anvisierten Bildungsziele steht zu vermuten, „dass die Traditionalität und die tiefe Religiosität die realistische Übertragung dieser Ziele blockieren. Traditionelle und autoritäre Erziehung ist nicht selten eine Erziehung, die nicht die Selbständigkeit und Verantwortlichkeit des Kindes zum Ziel hat, sondern die konsequente Einhaltung festgelegter Normen“ (Uysal 1998, S. 165). Fuhrer/Mayer betonen, dass im Prozess der Akkulturation türkischer Kinder insbesondere den Müttern eine Schlüsselrolle zufällt. Je stärker sie integriert sind, desto weniger autoritär fällt der Erziehungsstil in der Familie aus (vgl. Fuhrer/Mayer 2005). Auch andere kulturvergleichende Studien konnten zeigen, dass ein bestrafender Erziehungsstil weniger leistungsfördernd wirkt als z.B. die erlebte elterliche Unterstützung (vgl. Boehnke 1996). Allerdings gilt dies auch für Kinder aus Elternhäusern ohne Migrationshintergrund.

Auch aus diesem Grund beklagt Gogolin, dass Konstrukte über kulturelle Differenzen und deren Wirkung auf den Leistungsbereich nur unbefriedigende Interpretationsangebote für den geringen Bildungserfolg von Migrantenkindern liefern (vgl. Gogolin 2002, S.276). Indem derartige Erklärungsmuster zumeist auf eine Mangellage der Migranten verweisen und z.B. deren allgemeine Bildungsferne oder eine kulturbedingte Distanz zu bildungsrelevanten Institutionen betonen, gehen sie implizit von einer Defizithypothese aus (vgl. Bender-Szymanski/Hesse 
1987): Migrantenkinder befänden sich durch die Migrationssituation in einem ständigen „Kulturkonflikt“. Durch die Migration der Eltern bedingt, müssten sie ihr kulturelles Bezugssystem stetig wechseln, erführen so eine Veränderung ihrer Identität und schafften es nicht, die nötigen schulischen Anforderungsleistungen zu erbringen. Obwohl sich derzeit keine empirischen Belege für eine derartige Annahme finden lassen, wird das angenommene Zwischen-den-Stühlen-Sitzen zu einer wesentlichen „Ursache für die Schulschwierigkeiten von Immigrantenkindern" (Kronig 2003, S. 130). Andere Autoren widersprechen dieser These vehement und verweisen darauf, dass „die Persönlichkeitsentwicklung der Schüler durch die Migration der Eltern nicht gestört oder verändert wird" (Roebers/Mecheril/Schneider 1998, S. 734) und eine interkulturelle Lebenssituation allein keine Begründung für schulische Schwierigkeiten liefert. Meiner Einschätzung nach sind monokausale „Kulturkonflikt“-Konzepte insgesamt zu simpel, da sie die Sozialisationsbedingungen von Migrantenkindern lediglich unter dem Blickwinkel einer Einschränkung des Individuums und einer mangelnden Modernität betrachten und positive Auswirkungen auf die Identitätsentwicklung eines Menschen verneinen. Kultur verstanden als Orientierungssystem, das Wahrnehmen, Bewerten und Handeln steuert (vgl. Auernheimer 1994), betont demgegenüber eher die Potenziale, die sich aus der Zugehörigkeit zu einer bestimmten Gruppe ergeben. Insofern vertritt Preuss-Lausitz die auf Ulrich Beck zurückgehende These, dass Migrantenkinder dann erfolgreich sind, wenn sie nicht zwischen „deutscher Nachmoderne und ethnischer Tradition entscheiden müssen, sondern im Sinne einer Bastelbiografie beide Elemente miteinander verbinden können" (Preuss-Lausitz 2000, S. 26).

Neben familialen Prozessen der Akkulturation deuten eine Reihe von Untersuchungen darauf hin, dass die Ursachen für den geringen Bildungserfolg von Migrantenkindern auch in den von den Kindern besuchten Bildungsinstitutionen und dortigen Interaktionen begründet liegen. Leenen/Groß/Grosch z.B. weisen darauf hin, dass in institutionellen Kontexten „durchgängig die Gefahr von Wahrnehmungsverzerrungen, von Fehlzuschreibungen, Fehldiagnosen und unsachgemäßen Interventionen" aufgrund stereotyper Zuschreibungen besteht (Leenen/Groß/Grosch 2002, S. 81). Aus interaktionistischer Sicht ergeben sich deswegen insbesondere aus dem Zusammenspiel von Lehrererwartungen und Leistungsanforderungen an die Schüler Rückkoppelungseffekte. Negative Stereotype, die einen Zusammenhang von Gruppenzugehörigkeit und Begabung unterstellen, können die schulischen Leistungen der betroffenen Schülerinnen und Schüler beeinflussen. So setzen sich Menschen, die mit derartigen Stereotypen konfrontiert werden, selbst geringere Leistungsziele oder reagieren ängstlicher auf bevorstehende Testsituationen (vgl. Schofield 2006, S. 16, vgl. auch Kronig 2003, S. 130f). Effekte auf die Kompetenzen der schlechter eingeschätzten Schülerinnen und Schüler ergeben sich aber auch über das Verhalten der Lehrerinnen und Lehrer, die Schülerinnen und Schülern, von denen sie weniger erwarten, auch weniger 
emotionale Unterstützungsleistung bieten, diesen seltener und weniger detailliertes Feedback geben, seltener anspruchsvolle Arbeiten stellen, diese weniger loben, aber häufiger kritisieren. Da Lehrerinnen und Lehrer von Schülerinnen und Schülern mit Migrationshintergrund häufig weniger erwarten als von anderen Schülerinnen und Schülern, bekommen diese weniger Gelegenheit, Neues zu lernen (vgl. Schofield 2006, S. 66f).

Herwartz-Emden/Küffner stellen vor diesem Hintergrund einen Zusammenhang zwischen Selbstkonzept, Selbstwert und schulischem Erfolg von Migrantenkindern her. Dabei gehen sie davon aus, dass kulturspezifische Einflüsse auf das Selbstkonzept von Schülerinnen und Schülern mit Migrationshintergrund insofern einwirken, als diese Kinder Brüche in den schulischen Leistungen anders in ihr Selbstkonzept einbauen als autochthone Schülerinnen und Schüler und über eine Verankerung des Selbst in dominanteren Kontexten (Familie, Community) Brüche im Leistungsvermögen und inkonsistentes Selbsterleben besser verarbeiten können (vgl. Herwartz-Emden/Küffner 2006, S. 245). Erste Ergebnisse deuten hier darauf hin, dass Kinder mit Migrationshintergrund sich in ihrem Selbstwertgefühl lediglich im Bereich Schule von Kindern ohne Migrationshintergrund unterscheiden, diese aber ansonsten in ihrer Selbstkonzepteinschätzung sogar übertreffen können. Wie eng das Selbstkonzept verknüpft ist mit dem schulischen Leistungsvermögen, zeigt die Untersuchung von Brizic. Hier weisen Kinder mit einer hohen zweitsprachlichen Kompetenz auch ein höheres Selbstvertrauen auf als Kinder mit geringeren Kompetenzen in diesem Bereich (vgl. Brizic 2006, S. 261).

Neben Motivation und Selbstkonzept scheinen sich auch unterschiedliche Lernumgebungen unterschiedlich auf die Kompetenzen von Migrantenkindern auszuwirken. Schümer weist hier darauf hin, dass bezogen auf soziodemographische und leistungsbezogene Faktoren „Schulen unterschiedlicher Schulformen in verschiedener Hinsicht differenzielle Lern- und Leistungsumgebungen darstellen“ (Schümer 2001, S. 462). Damit ist gemeint, dass Schulen aufgrund ihrer institutionellen Arbeits- und Lernbedingungen und ihrer pädagogisch-didaktischen Traditionen ihren Schülern unterschiedliche schulmilieubedingte Entwicklungschancen eröffnen. Bezogen auf den Unterschied von Gymnasium und Hauptschule ergeben sich in Abhängigkeit von spezifischen Kompositionseffekten (z.B. dem Sozialstatus der Familien, dem Anteil von nicht vollzeitbeschäftigten Vätern oder dem Anteil von Kindern, die in ihrer Familie nicht deutsch sprechen) völlig konträre sozialökologische Profile beider Schulformen (vgl. Baumert/Stanat/Watermann 2006, S. 98f). Kompositionseffekte als Folge der Dreigliedrigkeit des Schulsystems führen vor allem in Hauptschulen zu erschwerten Arbeitsbedingungen. So kann Schümer drei Gruppen von Hauptschulen unterscheiden, die deutliche Leistungsunterschiede aufweisen. Die besten Ergebnisse erzielen Hauptschulen mit einem mittleren Migrantenanteil von 20\% und einem kleinen Anteil an Zuwandererfamilien, in denen nicht deutsch gesprochen wird, gefolgt von Hauptschulen mit einem mittleren Anteil von Migranten von 50\%, wovon $16 \%$ zu Hause kein 
Deutsch sprechen. Die geringsten Leitungen erbringen Schülerinnen und Schüler in Hauptschulen, in denen der Migrantenanteil 70\% beträgt und der Anteil der nicht deutsch sprechenden Familien bei 50\% liegt (vgl. Schümer 2001, S. 463f). Helmke kommt daher zu dem Schluss: „Eine ungünstige Klassenzusammensetzung setzt der Qualität des Unterrichts ebenso Grenzen, wie umgekehrt eine günstige Klassenzusammensetzung die Unterrichtsqualität und -effektivität fördern kann" (Helmke 2003, S. 54).

Ein weiterer Ansatz besteht darin, Bildungsbenachteiligungen systemtheoretisch auf den institutionellen Einfluss von Schule zurückzuführen. Gogolin (1994) macht z.B. auf den „monokulturellen Habitus“ der deutschen Schule aufmerksam, der einer Integration von Migrantenkindern in das deutsche Bildungssystem im Weg stehe. Bommes/Radtke (1993) führen dies entsprechend auf eine ,institutionalisierte Diskriminierung" durch das deutsche Schulsystem zurück. Diskriminierungseffekte entstehen dabei nicht nur aus subjektiven Handlungsformen der Unterrichtenden, sondern auch aus den der Schule in ihrer Eigenschaft als Organisation innewohnenden sozialen Prozeduren. Dabei unterscheiden die Autoren indirekte und direkte Formen der Diskriminierung: Direkte Formen der Diskriminierung beruhen auf der Verschiedenheit von Normen und Regeln, die auf einzelne Gruppen in der Gesellschaft angewendet werden (z.B. Vorbereitungsklassen nur für Migrantenkinder), während bei Formen der indirekten Diskriminierung die Anwendung gleicher Regeln und Normen ungleiche Chancen zur Folge hat (vgl. Bommes/Radtke 1993, S. 491). Im Sprachunterricht beispielsweise gelten für alle Kinder die deutschen Normen, unabhängig davon, welche Sprachkompetenz sie mitbringen. Gomolla betont, dass Diskriminierungen von Migrantenkindern auf der Basis der „Normalitätserwartungen in Bezug auf die Schul- und Sprachfähigkeit, wie sie deutschsprachigen, im weitesten Sinn christlich sozialisierten Mittelschicht-Kindern entsprechen“, erfolgen (Gomolla 2003, S. 105). Wirksam werden derartige Muster insbesondere bei Zuweisungsentscheidungen, z.B. beim Übergang nach der vierten Klasse.

Einige Untersuchungen verweisen daher auch auf die frühe Selektion innerhalb des deutschen Schulsystems und die hiermit einhergehenden Benachteiligungen bestimmter Bevölkerungsgruppen ${ }^{21}$. Beim Übergang von der Grundschule in eine weiterführende Schule wird z.B. die Empfehlungspraxis der Grundschullehrerinnen und -lehrern deutlich von sozialen Kontextfaktoren beeinflusst. Nach Gomolla wirken hier z.B. Formen der indirekten Diskriminierung, wenn von den Lehrerinnen und Lehrern vermutete mangelhafte häusliche Lernbedingungen und familiale Unterstützungspotenziale als Prognosekriterien für den Erfolg in den weiterführenden Schulen gewertet werden und deswegen eine Empfehlung in weniger prestigeträchtige Schulformen erfolgt (vgl. Gomolla 2003, S. 104). Die Erkenntnis, dass die „Messlatte“ für eine Gymnasialempfehlung bei gleicher kog-

21 Gleiches lässt sich im Übrigen auch für das Schulsystem in Österreich konstatieren (vgl. Furch 2006). 
nitiver Eignung für Kinder aus Migrantenfamilien deutlich höher liegt als für andere Schülerinnen und Schüler, bestätigen auch quantitative Studien (vgl. z.B. Lehmann/Peek 1997, S. 89, Dinkel/Luy/Lebok 1999, S. 370). Diskriminierende Wirkung bei der Übergangsempfehlung scheint dabei allerdings eher die Schichtzugehörigkeit und weniger der Migrationshintergrund $\mathrm{zu}$ entfalten (vgl. Schulz 2000, S. 477). Kristen hebt hervor, dass Schülerinnen und Schüler unterschiedlicher Herkunftsgruppen bei gleichen Testwerten von den Lehrerinnen und Lehrern ähnlich beurteilt werden und eine dementsprechende Empfehlung erhalten (vgl. Kristen 2006, S. 94). Im Gegensatz hierzu vermuten aber Lehmann/Peek für Hamburg, dass dort den Migrantenkindern von Seiten der Lehrkräfte „ein gewisser Bonus“ gegeben wird, da sie zwar insgesamt seltener eine Gymnasialempfehlung erhalten als deutsche Kinder, der für sie geltende Standard jedoch niedriger angesetzt wird. Offensichtlich wird erwartet, dass sie in der Sekundarstufe von einem fortschreitenden sprachlichen und sozialen Integrationsprozess profitieren (vgl. Lehmann/Peek 1997, S. 90).

\section{Bildungserfolg im Kontext von Mehrsprachigkeit}

Das bisher Ausgeführte verdeutlicht, dass Kinder mit Migrationshintergrund eine relevante Größe im deutschen Bildungswesen sind, ihr Bildungserfolg im Vergleich zu dem von Kindern der einheimischen Wohnbevölkerung jedoch eher gering ist. Zwar lässt sich auch im internationalen Kontext feststellen, dass Kinder eingewanderter Eltern zunächst geringere Qualifikationen erreichen als andere Kinder, Kinder der zweiten Generation verbessern jedoch in den meisten europäischen Ländern ihre Leistungen sowohl gegenüber den einheimischen Kindern als auch gegenüber den Kindern der ersten Generation. In Deutschland ist dieser Effekt nicht feststellbar (vgl. OECD 2007), hier sind vor allem die Kinder der zweiten Generation besonders erfolglos. Als Ursachen für diesen geringeren Bildungserfolg von Migrantenkindern werden in der einschlägigen Forschung sowohl familiale Faktoren, die im Wesentlichen auf die geringere Bildungsunterstützung fokussieren, individuelle Faktoren des Kindes bzw. der Jugendlichen selbst (Motivation, Selbstwert), aber auch systemimmanente Faktoren wie z.B. die vermutete institutionelle Diskriminierung diskutiert. Als wichtigste Voraussetzung für den schulischen Erfolg gilt aber insbesondere die Beherrschung der Unterrichtssprache.

In Verkennung der Lebensrealität vieler Migrantenkinder ging man lange Zeit implizit davon aus, dass diese bei Schuleintritt die Unterrichtssprache so gut beherrschen würden, dass sie problemlos am Unterricht teilnehmen könnten. Gesellschaftlich und auch schulpolitisch pflegte man die Vorstellung, dass sich eine sprachliche Assimilation an die Majoritätssprache nach einigen Generationen von selbst ergeben würde. Weitgehend ignoriert wurde dabei, dass viele Migrantenkinder zweisprachig aufwachsen und Deutsch für sie in den Lebensjahren vor der 
Einschulung nur eine marginale Rolle spielt, so dass dieses sprachliche Integrationsmuster ,aufgrund von demografischen, technischen und kulturellen Veränderungen seine Gültigkeit verloren hat - und zwar weltweit, nicht nur in Deutschland“ (Gogolin/Neumann/Roth 2003, S. 39f). Die Ursachen für die anhaltende und ungebrochene Loyalität und Vitalität der Einwanderersprachen sind vielfältig: Sie wird genährt durch ihre breite Präsenz in den heimischen Medien (z.B. Zeitschriften, Satellitenfernsehen) oder durch individuelle Möglichkeiten der Mobilität (z.B. Freizügigkeit innerhalb der EU, Erschwinglichkeit von Reisen in die Herkunftsländer). Für zweisprachig aufwachsende Kinder ist ihre Mehrsprachigkeit ein Schlüsselthema ihrer Sozialisation, das Konsequenzen für alle anderen Lebensbereiche hat (vgl. DJI 2000). Aus der Spracherwerbsforschung ist bekannt, dass das Aufwachsen mit zwei oder mehreren Sprachen jedes weitere sprachliche Lernen beeinflusst. Insofern wäre es an der Zeit, dass die monolinguale Institution Schule, sich stärker mit den Sprachen der Eingewanderten auseinandersetzt und bilinguale Konzepte stärker als bisher forciert würden.

\section{Literatur}

Auernheimer, G.: Struktur und Kultur. Über verschiedene Zugänge zu Orientierungsproblemen und -strategien von Migranten. In: Zeitschrift für Pädagogik, 40 (1994) 1, S. 29-42

Baumert, J./ Artelt, C.: Konzeption und Grundlage der Studie. In: Deutsches PISA-Konsortium (Hrsg.): PISA 2000: Basiskompetenzen von Schülerinnen und Schülern im internationalen Vergleich. Opladen 2001, S. 11-50

Baumert, J./ Schümer, G.: Schulformen als selektionsbedingte Lernmilieus. In: Deutsches PISA-Konsortium (Hrsg.): PISA 2000: Basiskompetenzen von Schülerinnen und Schülern im internationalen Vergleich. Opladen 2001, S. 454-467

Baumert, J./ Stanat, P./ Watermann, R.: Schulstruktur und die Entstehung differenzieller Lern- und Entwicklungsmilieus. In: Baumert, J./ Stanat, P./ Watermann, R. (Hrsg.): Herkunftsbedingte Disparitäten im Bildungswesen. Differenzielle Bildungsprozesse und Probleme der Verteilungsgerechtigkeit; vertiefende Analysen im Rahmen von PISA 2000. Wiesbaden 2006, S. 95-188

Bender-Szymanski, D./ Hesse, H.-G.: Migrantenforschung. Eine kritische Analyse deutschsprachiger empirischer Untersuchungen aus psychologischer Sicht. Köln 1987

Boehnke, K.: Is Intelligence Negligible? The Relationship of Family Climate and School Behaviour in a Cross-Cultural Perspective. Münster u.a. 1996

Bommes, M./ Radtke, F.-O.: Institutionalisierte Diskriminierung von Migrantenkindern. Die Herstellung ethnischer Differenz in der Schule. In: Zeitschrift für Pädagogik, 39 (1993) 3, S. 483-497 
Brizic, K.: Soziolinguistische Begleitstudie. In: Bundesministerium für Bildung, Wissenschaft und Kultur (Hrsg.): A kuci sprecham Deutsch. Sprachstandserhebung in multikulturellen Volksschulklassen: Bilingualer Spracherwerb in der Migration. Wien 2006, S. 253-306

Diehl, C.: Die Auswirkungen längerer Herkunftslandaufenthalte auf den Bildungserfolg türkisch- und italienischstämmiger Schülerinnen und Schüler. In: Haug, S./ Diehl, C.: Aspekte der Integration. Eingliederungsmuster und Lebenssituation italienisch- und türkischstämmiger junger Erwachsener in Deutschland. Wiesbaden 2005, S. 159-178

Diefenbach, H.: Schulerfolg von ausländischen Kindern und Kindern mit Migrationshintergrund als Ergebnis individueller und institutioneller Faktoren. In: BMBF (Hrsg.): Migrationshintergrund von Kindern und Jugendlichen: Wege zur Weiterentwicklung der amtlichen Statistik. Bonn, Berlin 2005, S. 43-54

Diefenbach, H./ Nauck, B.: Bildungsverhalten als „strategische Praxis". Ein Modell zur Erklärung der Reproduktion von Humankapital in Migrantenfamilien. In: Pries, L. (Hrsg.): Transnationale Migration. Baden-Baden 1997, S. 277-291

DJI Deutsches Jugendinstitut (Hrsg.): Wie Kinder multikulturellen Alltag erleben. München 2000

Fuhrer, U./ Mayer, S.: Familiäre Erziehung im Prozess der Akkulturation. In: Fuhrer, U./ Uslucan, H.-H.: Familie, Akkulturation und Erziehung. Migration zwischen Eigen- und Fremdkultur. Stuttgart 2005, S. 59-85

Gogolin, I.: Interkulturelle Bildungsforschung. In: Tippelt, R. (Hrsg.): Handbuch Bildungsforschung. Opladen 2002, S. 263-279

Gogolin, I./ Neumann, U./ Roth, H.-J.: Förderung von Kindern und Jugendlichen mit Migrationshintergrund. Gutachten im Auftrag der BLK. Bonn 2003

Gomolla, M.: Fördern und Fordern allein genügt nicht! Mechanismen institutioneller Diskriminierung von Migrantenkindern und -jugendlichen im deutschen Schulsystem. In: Auernheimer, G. (Hrsg.): Schieflagen im Bildungssystem. Opladen 2003, S. 97-112

Helmke, A./ Jäger, R. S. / Balzer, L./ Hosenfeld, I./ Ridder, A. / Schrader, F.-W.: Das Projekt MARKUS: Mathematik-Gesamterhebung Rheinland-Pfalz: Kompetenzen, Unterrichtsmerkmale, Schulkontext. Landau 2002

Herwartz-Emden, L.: Einwandererkinder im deutschen Bildungswesen. In: Cortina, K. S./ Baumert, J./ Leschinsky, A./ Mayer, K. U./ Trommer, L. (Hrsg.): Das Bildungssystem der Bundesrepublik Deutschland. Strukturen und Entwicklungen im Überblick. Harburg/Reinbek 2003, S. 661-709

Herwartz-Emden, L./ Küffner, D.: Schulerfolg und Akkulturationsleistungen von Grundschulkindern mit Migrationshintergrund. In: Zeitschrift für Erziehungswissenschaft, 9 (2006) 2, S. 240-254 
Klieme, E./ Beck, B.: Unterricht und Kompetenzerwerb in Deutsch und Englisch. Zentrale Befunde der Studie Deutsch-Englisch-SchülerleistungenInternational (DESI). Frankfurt/Main 2006. Abrufbar unter: http://www.dipf.de/desi/DESI_Zentrale_Befunde.pdf

Kristen, C.: Ethnische Unterschiede im deutschen Schulsystem. In: Aus Politik und Zeitgeschichte, (2003) B 21-22, S. 26-32

Kristen, C.: Ethnische Diskriminierung in der Grundschule? Die Vergabe von Noten und Bildungsempfehlungen. In: Kölner Zeitschrift für Soziologie und Sozialpsychologie, 58 (2006) 1, S. 79-97

Kristen, C./ Granato, N.: Bildungsinvestitionen in Migrantenfamilien. In: Bundesministerium für Bildung und Forschung (Hrsg.): Migrationshintergrund von Kindern und Jugendlichen. Wege zur Weiterentwicklung der amtlichen Statistik. Bonn 2005

Kronig, W.: Das Konstrukt des leistungsschwachen Immigrantenkindes. In: Zeitschrift für Erziehungswissenschaft, 6 (2003) 1, S. 126-14

Leenen, W. R./ Groß, A./ Grosch, H.: Interkulturelle Kompetenz in der Sozialen Arbeit. In: Auernheimer, G. (Hrsg.): Interkulturelle Kompetenz und pädagogische Professionalität. Opladen 2002, S. 81-102

Lehmann, R. H./ Peek, R./ Gänsfuß, R./ Husfeldt, V.: Aspekte der Lernausgangslage und der Lernentwicklung - Klassenstufe 9. Ergebnisse einer Längsschnittsuntersuchung in Hamburg. Hamburg 2002

Lehmann, R. H./ Hunger, S./ Ivanov, S./ Gänsfuß, R./ Hoffmann, E.: Aspekte der Lernausgangslage und der Lernentwicklung - Klassenstufe 11. Ergebnisse einer Längsschnittsuntersuchung in Hamburg. Hamburg 2004

Lehmann, R. H./ Ivanov, S./ Hunger, S./ Gänsfuß, R.: Untersuchung der Leistungen, Motivation und Einstellungen zu Beginn der beruflichen Ausbildung. Hamburg 2005

Lehmann, R. H./ Gänsfuß, R./ Peek, R.: Aspekte der Lernausgangslage und der Lernentwicklung von Schülerinnen und Schülern an Hamburger Schulen Klassenstufe 7. Bericht über die Untersuchung im September 1998 (unveröffentlichter Forschungsbericht). Hamburg 1999

May, P.: Orthographische Kompetenz und ihre Bedingungen am Ende der vierten Jahrgangsstufe. In: Bos, W./ Pietsch, M. (Hrsg.): KESS 4 - Kompetenzen und Einstellungen von Schülerinnen und Schülern am Ende der Jahrgangsstufe 4 in Hamburger Grundschulen. Münster 2006, S. 111-142

Nauck, B./ Diefenbach, H./ Petri, K.: Intergenerationale Transmission von kulturellem Kapital unter Migrationsbedingungen. In: Zeitschrift für Pädagogik, 44 (1998) 5, S. 701-722

Nauck, B./ Özel, S.: Erziehungsvorstellungen und Sozialisationspraktiken in türkischen Migrantenfamilien. In: Zeitschrift für Sozialisationsforschung und Erziehungssoziologie 2 (1986) 6, S. 285-312 
OECD: Education at a Glance. OECD Indicators. 2007. Abrufbar unter: http://www.oecd.org/dataoecd/4/55/39313286.pdf

Preuss-Lausitz, U.: Zwischen Modernisierung und Tradition. Bildungsprozesse heutiger Migrantenkinder. In: Die Deutsche Schule, 92 (2000) 1, S. 23-40

Ramm, G./ Prenzel, M./ Heidemeier, H./ Walter, O.: Soziokulturelle Herkunft: Migration. In: Deutsches PISA-Konsortium (Hrsg.): PISA 2003. Der Bildungsstand der Jugendlichen in Deutschland - Ergebnisse des zweiten internationalen Vergleichs. Münster 2004, S. 254-272

Ramm, G./ Walter, O./ Heidemeier, H./ Prenzel, M.: Soziokulturelle Herkunft und Migration im Ländervergleich. In: Deutsches PISA-Konsortium (Hrsg.): PISA 2003. Der zweite Vergleich der Länder in Deutschland - was wissen und können Jugendliche? Münster 2005, S. 269-298

Roebers, C. M./ Mecheril, A./ Schneider, W.: Migrantenkinder in deutschen Schulen. Eine Studie zur Persönlichkeitsentwicklung. In: Zeitschrift für Pädagogik, 44 (1998) 5, S. 723-736

Schofield, J. W.: Migrationshintergrund, Minderheitenzugehörigkeit und Bildungserfolg. Forschungsergebnisse der pädagogischen, Entwicklungs- und Sozialpsychologie. AKI-Forschungsbilanz 5. Berlin 2006. Abrufbar unter: http://www.wzb.eu/zkd/aki/files/aki_forschungsbilanz_5.pdf, vgl. auch Kronig 2003

Schroeder, D.: Bildungserfolgreich oder chancenlos? - Schulerfolg griechischer Migrantenkinder in der Bundesrepublik. In: Interkulturell, 1 (2002), S. $37-59$

Schümer, G.: Institutionelle Bedingungen schulischen Lernens im internationalen Vergleich. In: Deutsches PISA-Konsortium (Hrsg.): PISA 2000: Basiskompetenzen von Schülerinnen und Schülern im internationalen Vergleich. Opladen 2001, S. 411-427

Schulz, A.: Grundschule und soziale Ungleichheiten. Bildungsperspektiven in großstädtischen Regionen. In: Die Deutsche Schule, 92 (2000) 4, S. 464-479

Schwippert, K./ Bos, W./ Lankes. E.-M.: Heterogenität und Chancengleichheit am Ende der vierten Jahrgangsstufe im internationalen Vergleich. In: Bos, W./ Lankes, E.-M./ Prenzel, M./ Schwippert, K./ Walther, G./ Valtin, R. (Hrsg.): Erste Ergebnisse aus IGLU. Schülerleistungen am Ende der vierten Jahrgangsstufe im internationalen Vergleich. Münster 2003, S. 265-302

Uysal, Y.: Biographische und ökologische Einflussfaktoren auf den Schulerfolg türkischer Kinder in Deutschland. Eine empirische Untersuchung in Dortmund. Münster 1998 


\section{Nazife Toklu: Individuelle und institutionelle Dis- kriminierung muslimischer Kinder in deutschen Schulen}

Ein bisher in der Diskussion um die Einführung des islamischen Religionsunterrichts wenig beachtetes Thema ist das der Diskriminierung von muslimischen Kindern in deutschen Schulen. Sowohl durch meine ehrenamtliche Arbeit in einem Mentoren-Projekt an einer niedersächsischen Schule als auch durch meine derzeitige Arbeit bei Verikom (Verbund für interkulturelle Kommunikation und Bildung e.V.22 ), habe ich einen unmittelbaren Einblick in die diskriminierenden Strukturen dieser Institution erhalten. Der öffentliche Diskurs unterstellt muslimischen Kindern und Jugendlichen individuelles Versagen und eine mangelnde Integrationsbereitschaft. Das schulische Versagen muslimischer Kinder wird häufig auf ihre soziale Lage oder ihre Religion zurückgeführt, eine Religion, der das Stigma der Rückständigkeit angeheftet wird. Aus dieser Perspektive bleibt die Verantwortung der Gesellschaft und ihrer Bildungsinstitutionen für die Ausgrenzung von Kindern aus Familien mit Migrationshintergrund unsichtbar. Eine Vor- 
aussetzung dafür, dass die schulische Verantwortung für Diskriminierung sichtbar wird, ist die Eigenreflexion von Schule und Gesellschaft.

Im Folgenden möchte ich an die Diskurse erinnern in deren Zusammenhang die schulische Diskriminierung gesehen werden muss. Das Drama vom 11. September hat die bewusste und unbewusste Diskriminierung verstärkt, denn seit diesem Datum stehen Muslime besonders unter kritischer Beobachtung und werden vor allem aus sicherheitspolitischen Aspekten betrachtet. Dieses Attentat löste nicht nur in Deutschland eine verstärkte Islamophobie ${ }^{23}$ aus. Ein trauriger Beleg dieser Phobie sind die Studien von Heitmeyer - „Gruppenbezogene Menschenfeindlichkeit“ ${ }^{\text {(24 }}$ und Millamowitz-Moellendorf - „Was halten die Deutschen vom Islam“ 25 .

In seiner empirischen Langzeituntersuchung (2002 - 2012) hat Heitmeyer die menschenfeindlichen Einstellungen in der deutschen Bevölkerung untersucht, in der auch die Angst vor dem Islam zum Ausdruck kam (vgl. Heitmeyer 2005). Seine Befunde sind beunruhigend. In dieser Studie plädieren mehr als $25 \%$ der Bevölkerung dafür, Muslimen die Zuwanderung nach Deutschland zu untersagen. Mehr als $30 \%$ äußern, sie fühlten sich aufgrund der Anzahl der Muslime als Fremde im eigenen Land. $65 \%$ meinen, der Islam passe nicht in die westliche Kultur. Schließlich ist generelles Misstrauen gegenüber Muslimen bei etwa $65 \%$ vorhanden, und fast $50 \%$ sagen, sie hätten Probleme, in eine Gegend zu ziehen, in der viele Muslime leben.

$\mathrm{Zu}$ einem ähnlichen Ergebnis kommt die Untersuchung „Was halten die Deutschen vom Islam?“, die im Jahr 2003 im Auftrag der Konrad-Adenauer-Stiftung von Wilamowitz-Moellendorf durchgeführt wurde. Nach dieser Studie zweifelt fast die Hälfte der Bundesbürger (43\%) an der Toleranz des Islam, und $46 \%$ widersprechen der Ansicht, Islam und Christentum vertreten die gleichen Werte. Aussagen der Befragten wie: Deutschland sei ein christliches Land und muslimische Gebräuche hätten hier nichts zu suchen (vgl. Willamowitz-Moellendorf, 2003), sind für uns Muslime erschreckend und widersprechen dem Selbstverständnis einer pluralen demokratischen Gesellschaft.

Wie die beiden Studien verdeutlichen, nimmt die Ablehnung der islamischen Kultur in der deutschen Bevölkerung immer stärker zu. Der Islam ist seit dem Ende des Kalten Krieges und noch mehr seit dem 11. September zum neuen Feindbild geworden. In den Medien wird über den Islam immer häufiger nur im

23 Nach dem Soziologen und Friedensforscher Wilfried Graf dient die Islamophobie dazu, ein supranationales Europa, das einheitlich christlich geprägt sein soll, aufzubauen. Andere verstehen Islamophobie als den Antisemitismus des 21. Jahrhundert wie z. B. Andreas Hieronymus, Geschäftsführer des Institutes für Migrations- und Rassismusforschung, Hamburg.

24 Erste Ergebnisse der empirischen Langzeitbeobachtung unter:

http://www.berlin.de/imperia/md/content/lb-

$\mathrm{lkbgg} / \mathrm{bfg} /$ nummer20/03_heitmeyer.pdf?start\&ts=1239196349\&file=03_heitmeyer.pdf 25 http://www.kas.de/db_files/dokumente/arbeitspapiere/7_dokument_dok_pdf_1924.pdf 
Zusammenhang mit Terrorismus und Bedrohungsszenarien berichtet, er wird als fremd und gefährlich dargestellt. Die Berichterstattung über den Islam in Deutschland orientiert sich vornehmlich an Negativthemen. Der Islam mittlerweile zweitgrößte Religion in Deutschland wird häufig als „nicht integrierbar“ betrachtet, im schlimmsten Fall sogar als Herausforderung gegenüber dem säkularen Staat verstanden.

Die Muslime selbst kommen in den Medien indessen kaum zu Wort. Die Themen des deutsch-muslimischen Dialogs werden von der Mehrheitsgesellschaft vorgegeben, in der Regel findet er dann statt, wenn von den Muslimen und ihren Organisationen Abgrenzung von Ehrenmorden, Zwangsheirat, Terrorismus, innerfamiliärer Gewalt etc. gefordert wird.

Das in der bundesrepublikanischen Öffentlichkeit vorherrschende Islambild macht auch nicht vor den Schulpforten halt. Das verinnerlichte Islambild prägt bei vielen Lehrerinnen und Lehrern das pädagogische Alltagshandeln. Sie tragen ihr Klischee aus der öffentlichen Debatte in die Klassenzimmer: So werden Ehrenmorde in der Diskussion mit dem Koran in Verbindung gesetzt, obwohl er hierzu nichts aussagt. Er sagt auch nichts zur Zwangsheirat und den zuvor genannten Themen aus. Hierbei wird der Islam häufig als eine kriegerische, unterdrückende, frauenfeindliche, mittelalterliche und intolerante Religion dargestellt. In vielen Fällen mündet die Umsetzung dieser Klischees in individuelle Diskriminierung um.

\section{Fallbeispiele schulischer Diskriminierung}

Meine Aussage möchte ich mit einigen Beispielen verdeutlichen:

Irakkrieg

Im Rahmen meiner Arbeit für „,verikom“ wurde mir von einem zwölfjährigen muslimischen Schüler berichtet, dass sein Lehrer im Fach Werte und Normen hier stand der Islam gerade auf dem Lehrplan - ihn zielgerichtet fragte, ob es sich beim Irakkrieg um einen Befreiungsakt oder um eine Okkupation handeln würde. Der Schüler war verblüfft und antwortete mit der Gegenfrage: „Seit wann wird ein Land mit einem Krieg befreit?“. Der Lehrer ließ diese Frage unbeantwortet, stellte jedoch in Aussicht, die Frage nach dem Unterricht zu beantworten. Nach dem Ende der Unterrichtseinheit ging der Schüler zum Lehrer und wollte die Antwort erfahren. Der Lehrer wich auch diesmal aus und zwar mit der Begründung, dass er eine höhere Schulklasse zu unterrichten habe und keine Zeit hätte, die Frage zu beantworten. Warum wurde diese Frage nicht an die gesamte Klasse gerichtet, sondern nur an den einzigen muslimischen Schüler des Klassenverbands und was hat der Irakkrieg ursächlich mit dem Islam zu tun? 
Kopftuchdebatte

Nach der hitzigen Kopftuchdebatte wurden eine muslimische Schülerin und ein Schüler in einem niedersächsischen Gymnasium in der ersten Stunde nach der Einschulung in die sechste Klasse von der Mathematikfachlehrerin vor der gesamten Klasse angesprochen, dass sie in ihrem Unterricht keinerlei religiöse Symbole dulden werde. Zu den religiösen Symbolen gehören für sie das Kopftuch und die Takke eine weiße, gehäkelte Kopfbedeckung für muslimische Männer, die diese beim Gebet aufsetzen. Sie ermahnte die beiden türkischen Kinder ausdrücklich, dass sie nicht auf die Idee kommen sollten, so etwas zu tragen. Ich erwähne dieses Beispiel aus einem besonderen Grund: Dieses Gymnasium hält in seiner Präambel fest, dass es sich als Ort der Gewaltfreiheit versteht, an dem Schülerinnen und Schüler, Lehrerinnen und Lehrer in einer Gemeinschaft angstfrei miteinander arbeiten und lernen können. Die Schule hat den Titel „Schule ohne Rassismus“ verliehen bekommen und möchte nach Eigenaussage diesem Titel gerecht werden. Ausdrücklich wird in der Präambel erwähnt, dass die Grund- und Menschenrechte und demokratische Prinzipien für alle gelten. Bloßstellendes Verhalten von Lehrkräften an Schulen insbesondere an „Schulen ohne Rassismus“ ist nicht entschuldbar und nicht hinnehmbar!

Verbot von Kopfbedeckungen:

Ein letztes Beispiel für schulische Diskriminierung stammt aus BadenWürttemberg. Laut einem Elternbrief des Robert-Mayer-Gymnasiums in Heilbronn von März 2006 26, der die Eltern über eine Schulkonferenz informiert, wurden an der Schule alle Arten von Kopfbedeckungen für Schüler und Schülerinnen verboten. In dem Elternbrief heißt es, dass in der Schulkonferenz vom April 2004 die Schülersprecher, das Kollegium und die Vertreter der Elternschaft gemeinsam „befürwortet und beschlossen“ hätten, dass an diesem Gymnasium „auch weiterhin keinerlei Kopfbedeckungen gestattet sind“. Die Schule „wünsche sich eine dem Unterricht angemessene Kleidung“. Auf Anfrage des deutschtürkischen Juristen Ekrem Senol und Betreibers der Internetseite jurblog.de bestätigte die Schule, dass dieses Verbot auch das aus religiösen Gründen getragene Kopftuch betreffe. Eine Schülerin mit Kopftuch gebe es bisher an der Schule nicht. Damit versucht die Schule per Hausordnung die grundrechtlich geschützte Religionsfreiheit von vornherein einzuschränken. Ein „Problem“ mit Kopftuch tragenden Mädchen wird es an der Schule wohl nicht geben, da diese wahrscheinlich mit Hinweis auf diese Regelung erst gar nicht auf die Schule aufgenommen werden.

Ich verzichte hier auf das weitere Aufzählen von ähnlichen Beispielen individueller und kollektiver Diskriminierung. Ich gehe hier auch nicht auf die Diskri-

26 Vgl. Ekrem Senol, http://www.jurblog.de/2006/06/28/schule-verbietet-alle-arten-vonkopfbedeckungen 
minierung der Schülerinnen und Schüler untereinander ein, vielmehr möchte ich aber auf eine weitere Art der Diskriminierung hinweisen, und zwar auf die der institutionellen Diskriminierung.

Strukturelle Diskriminierung bedeutet, dass Menschen aufgrund sozial relevanter Merkmale wie u.a. der Ethnie, der Religion oder Weltanschauung in verschiedenen Lebensbereichen systematisch anders behandelt werden. Strukturelle Diskriminierung beruht auf eingespielten und dauerhaften, oft formalisierten und explizit geregelten institutionellen Praktiken.

Institutionelle Diskriminierung im Bereich Schule zeigt, dass die Rahmenbedingungen von Schule als gesellschaftliche Institution und als System die Elitenbildung statt der Integration Benachteiligter, hier insbesondere Kinder und Jugendliche mit Migrationshintergrund, fördert.

Institutionelle Diskriminierung im Bereich Schule zeigt, dass die nicht die Integration Benachteiligter, hier insbesondere Kinder und Jugendliche mit Migrationshintergrund, fördert. Institutionelle Diskriminierung spiegelt sich zum Beispiel darin wider, dass Kinder aus Migrantenfamilien von der Zurückstellung vom Schulbesuch und der Einweisung in den Schulkindergarten überproportional betroffen sind. In Sonder- und Hauptschulen sind sie ebenfalls deutlich überrepräsentiert. Bei Kindern und Jugendlichen mit Migrationshintergrund, die zudem noch dem islamischen Glauben angehören, werden als Ursache von Lernversagen vielfach der Islam und Koranschulen angegeben („Fast alle unsere türkischen Sonderschulfälle, da steckt die Koranschule dahinter" Zitat eines Schulleiters)(vgl. Gomolla/Radtke 2002, S. 205). Selbst bei guten Noten wird ihnen vermehrt der Besuch der Real- oder Hauptschule empfohlen, und zwar unter anderem mit der Begründung, dass ohne perfekte Deutschkenntnisse kein Erfolg auf dem Gymnasium möglich sei (vgl. Terkessidis 2004, S. 154 ff). Leider werden Potenziale wie Zweisprachigkeit nicht erkannt und gewürdigt, sondern vielmehr werden häufig nur Defizite herausgestellt, die bei den Schülerinnen und Schülern langfristig zu einer Demotivierung führen. Internationale Leistungsstudien belegen hier sehr deutlich, dass es dem deutschen Schulsystem nicht gelingt, Kinder mit Migrationshintergrund ausreichend zu fördern. Man könnte sagen, dass die dort festgestellten geringeren Kompetenzen der Kinder mit Migrationshintergrund eine direkte Folge der institutionellen Diskriminierung sind. Zur Verdeutlichung möchte ich hier einige zentrale Ergebnisse folgender Studien anführen:

\section{PISA-Studien}

Die Pisa-Studie 2003 sagt aus, dass es in der Bundesrepublik eine unverhältnismäBig hohe Wechselbeziehung zwischen mangelnder Lesefähigkeit und Migrationshintergrund gibt. Das bedeutet, dass die Chancen für Schülerinnen und Schüler aus Migrantenfamilien deutlich geringer sind, eine ausgezeichnete Lesefähigkeit zu erwerben. Somit sind für die jungen Menschen mit Migrationshintergrund auch 
die Chancen geringer, eine höhere Schulbildung zu erlangen und später auch zu studieren.

Internationale Grundschul-Lese-Untersuchung (IGLU)

In ihr wurde 2003 festgestellt, dass die Gymnasialempfehlung nach der Grundschule in den seltensten Fällen aufgrund der von einem Kind erbrachten Leistung erfolgt, sondern oftmals an die Herkunft des Kindes gekoppelt ist (vgl. Terkessidis 2004, S. 156). Kinder aus unteren sozialen Schichten und Kinder von Migranten müssen z.B. mehr leisten, um eine Gymnasialempfehlung zu erhalten als Kinder aus höheren sozialen Schichten.

Schwerpunktprogramm FABER „Folgen der Arbeitsmigration für Bildung und Erziehung" der Deutschen Forschungsgemeinschaft

„Diese Studie macht deutlich, dass im Hinblick auf das noch immer von einem nationalen Selbstverständnis geprägte Bildungssystem eine grundlegende Neuorientierung im Blick auf die strukturelle und inhaltliche Organisation etwa von Schule sowie die Ausbildung von pädagogischen Fachkräften erforderlich ist. Andernfalls wird das Bildungssystem seiner Aufgabe in einer demokratischen Gesellschaft nicht gerecht und trägt zur (Re-)Produktion von Diskriminierungsund Ungleichheitsverhältnissen bei““ (Mar Castro-Varele/Mecheril 2006, S. 415.)

\section{UNICEF-Studie}

Die UNICEF-Studie hat durch Vergleiche der Bildungschancen in den OECDLändern bestätigt, dass das Schulsystem der Bundesrepublik Deutschland stark nach sozialer Herkunft selektiert“" Mar Castro-Varele/Mecheril 2006 S. 415.

Die vorgenannten Studien belegen unabhängig voneinander, dass das deutsche dreigliedrige Schulsystem sehr stark leistungsorientiert ausgerichtet ist und hier nicht die breite Förderung aller Schülerinnen und Schülern im Fokus hat. Sie werden in ihren Leistungen und nach ihrem soziokulturellen Herkunftsmilieu sehr früh den verschiedenen Schulformen zugeordnet. Diese Selektionspraxis steht einer nachhaltigen und wirkungsvollen Förderung von Schülerinnen und Schülern mit Migrationshintergrund entgegen, denn dieses System korrigiert nicht die Lernprobleme der Schülerinnen oder Schüler im Unterricht, sondern gibt diese an andere Schulformen mit geringeren Leistungsanforderungen ab (vgl. Kiper 2006, S. 290f.) Das bundesdeutsche Bildungssystem ist der Herausforderung, wirkliche Chancengleichheit zu realisieren, leider bisher kaum nachgekommen.

Alle Studien, insbesondere die PISA-Studien haben die ungünstigen Rahmenbedingungen für Kinder und Jugendliche mit Migrationshintergrund im deutschen Bildungssystem öffentlich zur Diskussion gestellt. Ich sehe deshalb verhaltend optimistisch in die Zukunft und hoffe, dass es langfristig zu einer umfassenden Reform des deutschen föderalen Bildungssystems kommt, die auf Herstellung von 
Chancengleichheit abzielt. Schule muss zu einer Schule in einem Einwanderungsland werden. Bereits von der Kultusministerkonferenz beschlossene interkulturelle Aus- und Fortbildungsprogramme für Lehrerinnen und Lehrer müssen umgesetzt werden. Die Aufhebung von Benachteiligung und Diskriminierung ist nicht allein durch pädagogische Programme zu erreichen, sie bedarf einer politischen und gesellschaftlichen Anerkennung. Die Einführung des Islam als Religionsunterricht ist hier ein kleiner Schritt in die richtige Richtung und zeigt, dass nunmehr nach fast einem halben Jahrhundert realisiert wird, dass der Islam ein Bestandteil der deutschen Gesellschaft ist. Weiterhin hoffe ich, dass der Schulversuch zum islamischen Religionsunterricht in Niedersachsen sich aus der Erprobungsphase verstetigt und flächendeckend eingeführt werden kann.

\section{Literatur}

Bax, D. : In: Taz: 29. / 30 Juli 2006: Die Integration der Muslime hat gerade erst begonnen.

Gomolla, M./Radtke, F.-O.: Institutionelle Diskriminierung. Die Herstellung ethnischer Differenz in der Schule., Opladen 2002

Heitmeyer,W.: Gruppenbezogene Menschenfeindlichkeit - Empirische Langzeitbeobachtung menschenfeindlicher Einstellungen in der Bevölkerung, Universität Bielefeld. In: Deutsche Zustände, Folge 3. Frankfurt, 2005

Kieser, A./Stang, A./Eckl, W./Vasseur, G.: Maßnahmen zur Vermeidung von Diskriminierung in Schulen und im beruflichen Alltag in Frankreich. Berliner Institut für Vergleichende Sozialforschung. Berlin 2005

Kiper,H.: Interkulturelles Lehren und Lernen in Unterrichtsfächern der Primarstufe in: Leiprecht,R./ Kerber. A. (Hrsg.): Schule in der Einwanderungsgesellschaft. Schwalbach 2006, S.290-302

Leiprecht, R./Kerber, A. (Hrsg.): Schule in der Einwanderungsgesellschaft. Ein Handbuch. In der Reihe Politik und Bildung - Band 38. Schwalbach 2006

Lützel, A./Eckl, W.: Rassismus und Diskriminierung in den beruflichen Schulen Das Beispiel Berlin. In: Studie im Rahmen des Xenos-Projekts „Vermittlung gefährdeter Jugendlicher in Betriebe in Berlin und Brandenburg mit wissenschaftlichen und begleitenden Forschungsarbeiten“. Berliner Institut für Vergleichende Sozialforschung. Berlin 2005

Mächler, S./Stang, A./Meyer, I./Pfohmann, S.: Maßnahmen zur Vermeidung von Diskriminierung in Schulen und im beruflichen Alltag in Großbritannien. Berliner Institut für Vergleichende Sozialforschung. Berlin 2005

Mar Castro Varele, M./ Mecheril, Paul: Minderheitenangehörige und "professionelles Handeln". Anmerkungen zu einem unmöglichen Verhältnis in: Leiprecht, R./ Kerber, A. (Hrsg.): Schule in der Einwanderungsgesellschaft. Ein Handbuch. Schwalbach 2006, S. 406-419 
Terkessidis, M.: Die Banalität des Rassismus. Migranten zweiter Generation entwickeln eine neue Perspektive. Bielefeld 2004

Wilamowitz-Moellendorf, U. von: Was halten die Deutschen vom Islam - Ergebnis einer Umfrage, Sankt Augustin 2003 

Auf dem Weg zum Islamischen Religionsunterricht 


\section{Heidemarie Ballasch: Schulversuch „Islamischer Religionsunterricht" in Niedersachsen. Auf dem Weg zum Islamischen Religionsunterricht als or- dentliches Unterrichtsfach}

Im schulischen Kontext kann das Verhältnis zum Islam als eine Schlüsselfunktion in der Integration muslimischer Schülerinnen und Schüler gesehen werden. Die Einführung von islamischem Religionsunterricht als ordentliches Unterrichtsfach ist daher Thema in vielen Bundesländern.

In unterschiedlichen Modellen werden Wege erprobt, die das Ziel verfolgen, islamischen Religionsunterricht in den Kanon der ordentlichen Lehrfächer aufzunehmen. Da in Niedersachsen ein breiter politischer und gesellschaftlicher Konsens darüber besteht, islamischen Religionsunterricht anzubieten, konnten rechtliche und organisatorische Schwierigkeiten zugunsten des Schulversuchs überwunden werden. Die niedersächsische Landesregierung sah und sieht es als ihre Verpflichtung an, das grundgesetzlich verbriefte Recht auf konfessionellen Religionsunterricht allen Schülerinnen und Schülern zu ermöglichen, nicht nur christlichen, jüdischen oder orthodoxen Schülerinnen und Schülern, sondern insbesondere auch muslimischen Schülerinnen und Schülern. Mit der Einführung von „Islamischem Religionsunterricht" wird daher das Recht auf religiöse Bildung anerkannt. 


\section{Rechtliche Voraussetzungen}

Der Schulversuch stützt sich politisch auf die Regierungserklärung des Niedersächsischen Ministerpräsidenten vor dem Niedersächsischen Landtag am 24. Oktober 2001. In der Regierungserklärung wurde deutlich zum Ausdruck gebracht, dass das Land alle Anstrengungen unternehmen will, die Entwicklung von „Parallelgesellschaften“ zu vermeiden, zugleich aber „eigene kulturelle Bindungen und Identitäten“ achten und wertschätzen wird. Wörtlich heißt es:

„Wir werden dabei auch die Möglichkeit zur Erteilung eines islamischen Religionsunterrichts in deutscher Sprache, aber unter staatlicher Verantwortung ermöglichen. Im Zentrum muss dabei ein integrativer Unterricht stehen, der die Beziehungen herstellt zwischen der eigenen Religion und Kultur und unserem freibeitlichen und sozialen Menschen- und Gesellschaftsbild."

Die Einführung von islamischem Religionsunterricht als ordentliches Unterrichtsfach ist an Voraussetzungen gebunden. Islamischer Religionsunterricht muss - wie jeder andere Unterricht auch - inhaltlich den Verfassungsansprüchen und prinzipien und dem darauf basierenden Bildungsauftrag von Schule entsprechen. Für einen Unterricht, der nicht den Maßstäben der Verfassung entspricht, gibt es in der Schule keine Legitimation und keinen Platz. Für die Erteilung von konfessionellem Religionsunterricht gilt:

- Die Erteilung des Religionsunterrichts ist staatliche Aufgabe und Angelegenheit, sein Gegenstand hingegen sind die Glaubensinhalte der jeweiligen Religionsgemeinschaft.

- Zur Durchführung des Religionsunterrichts bedarf der Staat eines für die Religionsgemeinschaft autorisierten und legitimierten Ansprechpartners.

- Lehrkräfte, die den Religionsunterricht erteilen, müssen in ihrer wissenschaftlichen und pädagogischen Ausbildung eine vergleichbare Befähigung nachweisen wie die Lehrkräfte, die eine Lehrbefähigung für ein anderes ordentliches Unterrichtsfach besitzen.

- Die Schulbehörde erlässt die Lehrpläne (Kerncurriculum) für den Religionsunterricht und genehmigt Lehrbücher im Einvernehmen mit der Religionsgemeinschaft.

- Religionsunterricht als ordentliches Unterrichtsfach unterliegt der Kontrolle des Staates, wird benotet und hat Einfluss auf die Versetzung. Die Unterrichtssprache muss Deutsch sein, da dieser Unterricht von Schülerinnen und Schülern unterschiedlicher Herkunftssprachen besucht wird.

Diese Voraussetzungen sind in Niedersachsen für den evangelischen, katholischen, jüdischen und orthodoxen Religionsunterricht gegeben. Der entsprechende Religionsunterricht ist einzurichten, wenn an einer Schule eine Lerngruppe von mindestens zwölf Schülerinnen und Schülern eines Glaubens gebildet werden kann. Erfolgt die Abmeldung vom Religionsunterricht, ist das Fach Werte und 
Normen zu belegen. Religionsunterricht ist Pflichtfach von der Grundschule bis zum Abitur.

Das Fach Werte und Normen ist Pflichtfach vom Schuljahrgang 5 bis 12 und wird nicht in der Grundschule erteilt. Für den islamischen Religionsunterricht sind die rechtlichen Bedingungen derzeit nicht erfüllt. Daher kann dieses Unterrichtsangebot nur im Rahmen eines Schulversuchs erfolgen. Rechtsgrundlage für den Schulversuch „Islamischer Religionsunterricht" mit dem Ziel, deutschsprachigen „Islamischen Religionsunterricht“ zu erproben, ist $\$ 22$ Niedersächsisches Schulgesetz.

Der Schulversuch „Islamischer Religionsunterricht“ ist zeitlich befristet und beschränkt sich auf die Schulform Grundschule. Weil es in dieser Schulform kein alternatives Fach zum Religionsunterricht gibt, müssen die Schülerinnen und Schüler, sofern sie nicht am evangelischen oder katholischen Religionsunterricht teilnehmen, ein Förder- oder Betreuungsangebot wahrnehmen oder haben sogar eine „Freistunde“.

\section{Zielsetzung des Schulversuchs}

Nicht nur weltweit verzeichnet der Islam innerhalb der Weltreligionen die höchsten Zuwachsraten, sondern auch in niedersächsischen Schulen: Die Anzahl der Schülerinnen und Schüler evangelischen Bekenntnisses ist in den letzten 20 Jahren um gut $10 \%$ (von $69 \%$ auf $58 \%$ ) gesunken, die Anzahl der Schülerinnen und Schüler katholischen Bekenntnisses um $3 \%$ (von knapp $21 \%$ auf ca. $18 \%$ ). Die Anzahl der Schülerinnen und Schüler islamischen Glaubens hingegen hat sich verdoppelt: von $2,4 \%$ auf über $5 \%$.

Die Dringlichkeit und Notwendigkeit, auf diese Tatsache mit pädagogischen Konzepten zu reagieren, bilden eine entscheidende Begründung für den Schulversuch. Für die drittgrößte Glaubensgemeinschaft verfolgt die Landesregierung mit dem Schulversuch „Islamischer Religionsunterricht“ das Ziel, ein Religionsunterrichtsangebot zu ermöglichen, das den verfassungsmäßigen und schulgesetzlichen Voraussetzungen entspricht. Der Schulversuch leistet zugleich einen wichtigen Beitrag zu einer positiven Wahrnehmung des Islam in der Öffentlichkeit, da mit diesem Schritt der Islam im schulischen Kontext eine Gleichstellung zu den christlichen Religionen erfährt.

Der Schulversuch verfolgt das Ziel,

- den Schülerinnen und Schülern Wissen und Kenntnisse über ihre eigene und über andere Religionen zu vermitteln,

- die Schülerinnen und Schüler zu einer mündigen Glaubensentscheidung zu befähigen,

- einen Beitrag zur Integration zu leisten und damit gesellschaftlich parallelen Strukturen entgegenzuwirken. 
Um abzusichern, dass mit dem Schulversuch die angestrebten Ziele erreicht werden und unerwünschte und unerwartete Ergebnisse rechtzeitig bemerkt werden, wurde der Schulversuch wissenschaftlich begleitet.

\section{Religionsunterricht - nicht Islamkunde}

Mit dem Schulversuch hat Niedersachsen den Sprung von der abstrakten Ebene der Debatte um den Islamunterricht auf die handelnde Ebene des Unterrichts gewagt.

Im Gegensatz zu anderen Bundesländern - Nordrhein-Westfalen bietet das Fach "Islamkunde" an, das objektive Informationen über den Islam, aber keine Erfahrung der eigenen Religion zum Ziel hat - hat sich Niedersachsen bewusst für das Fach Religionsunterricht entschieden, in dem aus dem Glauben heraus wichtige Sinnfragen mit den Schülerinnen und Schülern erörtert werden sollen. Im Religionsunterricht ist der Islam sowohl Gegenstand der Auseinandersetzung als auch der subjektiven Erfahrung; informierende Elemente finden darin ebenso ihren Platz wie religiöse Werteerziehung.

Der Religionsunterricht ist neben dem Leben in der Familie und in der jeweiligen Gemeinde und Glaubensgemeinschaft ein maßgeblicher Ort, an dem existenzielle Fragen, wie sie religiöse Fragen sind, behandelt werden können, die viele junge Menschen beschäftigen: Woher komme ich, wohin gehe ich, worin liegt der Sinn meines Lebens?

Für die niedersächsische Landesregierung ist Religion kein Randfach. Es wird immer deutlicher, wie wichtig ein Unterricht ist, der sich zu dem Bekenntnis bekennt und das Bekenntnis zum Gegenstand des Diskurses macht. Gerade ein kritischer Religionsunterricht bewirkt, dass Schülerinnen und Schüler darüber nachdenken, wie sie sich im Glauben positionieren. Dabei wird davon ausgegangen, dass die Befähigung der Schülerinnen und Schüler, persönliche Entscheidungen begründet treffen zu können, es ihnen ermöglicht, die Entscheidungen anderer tatsächlich nachvollziehen und tolerieren zu können.

\section{Der Runde Tisch}

Niedersachsen befindet sich auf dem Weg zum „Islamischen Religionsunterricht“, da die verfassungsgemäßen Voraussetzungen bisher nicht erfüllt sind. Dem Islam sind kirchenähnliche Strukturen - und damit mitgliedschaftliche Strukturen fremd und daher gibt es keinen legitimierten Ansprechpartner der Religionsgemeinschaft auf muslimischer Seite und keinen Bekenntnisnachweis wie bei den christlichen Religionsgemeinschaften.

Durch die Einberufung eines „Runden Tischs Islamischer Religionsunterricht“ hat die Landesregierung versucht, die gegenwärtigen Schwierigkeiten der muslimi- 
schen Gemeinschaften mit den Anforderungen des Art. 7 Abs. 3 GG für den Schulversuch zu überwinden. Der Runde Tisch, an dem die relevanten Organisationen und Vereine der Muslime in Niedersachsen beteiligt waren und sind, ist für die Dauer des Schulversuchs Ansprechpartner des Landes in den zentralen Glaubensfragen des Islam.

Der Nachweis der Zugehörigkeit zur Glaubensgemeinschaft erfolgt ebenfalls für die Dauer des Schulversuchs durch die Anmeldung zum Unterricht durch die Erziehungsberechtigten. Am Runden Tisch arbeiten regelmäßig Vertreter bedeutender islamischer Verbände, Gemeinschaften oder Zentren Niedersachsens mit, sodass die verschiedenen islamischen Glaubensgemeinschaften angemessen vertreten sind. Die Aleviten, die zu Beginn am Runden Tisch vertreten waren, haben mittlerweile einen Antrag auf einen eigenständigen alevitischen Religionsunterricht in Niedersachsen gestellt.

Die Mitarbeit am Runden Tisch verläuft offen und engagiert, denn alle Beteiligten wollen das Ziel, den islamischen Religionsunterricht als ordentliches Unterrichtsfach einzuführen, auf keinen Fall gefährden. Auch wenn es mitunter zu Irritationen kommt, ist es bisher immer gelungen, das Gespräch weiterzuführen und den Dialogpartner zu verstehen.

Um die Zusammenarbeit zwischen Landesregierung und muslimischen Vereinen zu vertiefen, wurde dem Runden Tisch das Recht eingeräumt, zwei islamischtheologische Expertinnen zu benennen, die u.a. in der begleitenden Fortbildung oder bei der Erarbeitung von Unterrichtsmaterialien beratend tätig sind.

\section{Rahmenrichtlinien des Schulversuchs}

In Übereinstimmung mit dem Runden Tisch wurden die derzeit geltenden Rahmenrichtlinien entwickelt. Diese werden nun natürlich im Austausch mit dem Runden Tisch daraufhin überprüft, inwieweit sie der unterrichtlichen Arbeit dienlich sind und wie sie weiter zu entwickeln sind. Außensichtweisen (vgl. Irka Mohr 2008) geben für die Evaluation der Rahmenrichtlinien wertvolle Hinweise.

Während in den alten Rahmenrichtlinien verbindliche Themen die Unterrichtsgrundlage darstellten, soll zukünftig wie im evangelischen und katholischen Religionsunterricht auch von Leitfragen ausgegangen werden, die das Fach inhaltlich strukturieren. Zukünftig soll also im Lehrplan nicht nur beschrieben werden, was die Schülerinnen und Schüler wissen sollen, sondern es sollen auch die Prozeduren markiert werden, wie mit dem Wissen umgegangen werden soll. Für die Schülerinnen und Schüler soll klar werden, ob sie beispielsweise einen Inhalt wahrnehmen, beschreiben, verstehen oder deuten sollen. Es wird im neuen Lehrplan also nicht allein um das Wissen gehen, das im Unterricht vermittelt werden soll, sondern um die Anwendung des Wissens, das Können. 


\section{Zur aktuellen Situation}

Der Schulversuch befindet sich mit dem Schuljahr 2008/2009 im sechsten Schulversuchsjahr. An acht Schulstandorten wurde der Schulversuch gestartet. Jedes Jahr wurde der Schulversuch ausgeweitet, sodass mittlerweile an insgesamt 29 Schulstandorten „Islamischer Religionsunterricht" für über 1.600 muslimische Schülerinnen und Schüler angeboten wird. Der Unterricht von zwei Wochenstunden wird in deutscher Sprache von muttersprachlichen Lehrkräften jetzt herkunftssprachlichen Lehrkräften islamischen Glaubens erteilt, die bereits im Rahmen des herkunftssprachlichen Unterrichts Erfahrungen in dem Bereich der „Themen der religiösen Landeskunde“ gesammelt haben. Der Schulversuch „Islamischer Religionsunterricht" wurde bisher jedes Jahr verlängert und auf weitere Schulen ausgeweitet, sofern geeignete Religionslehrkräfte zur Verfügung standen. Mit gegenwärtigem Stand dauert der Versuch bis zum 31.07.2012.

Da es bisher in Deutschland keine ausgebildeten Lehrkräfte für das Fach „Islamischer Religionsunterricht" gibt, muss ein pragmatischer Weg eingeschlagen werden, um die Lehrkräfte auf die neue Aufgabe vorzubereiten. Eine zusätzliche Qualifikation in religionspädagogischen und didaktisch-methodischen Fragen erhalten die Lehrkräfte durch eine Fortbildungsmaßnahme des Niedersächsischen Landesamtes für Lehrerbildung und Schulentwicklung (NiLS).

Die Ausbildung von Lehrkräften, die islamischen Religionsunterricht erteilen, ist in allen Bundsländern, die Schulversuche eingerichtet haben, eine Herausforderung. Während in einigen Bundesländern Islamwissenschaftler den Unterricht erteilen, die sich pädagogisch qualifizieren müssen, werden in Niedersachsen die Lehrkräfte religionsdidaktisch qualifiziert.

Beide Wege zeigen, dass kreative Übergangslösungen gefunden wurden, um den fachwissenschaftlichen und pädagogischen Ansprüchen, die grundsätzlich an jedes Unterrichtsfach zu stellen sind, zu genügen.

\section{Fortbildung der Lehrkräfte}

„Aller Anfang ist schwer“, so formulierte es eine Lehrkraft, die in den Schulversuch eingebunden ist. Die Lehrkräfte, die den islamischen Religionsunterricht erteilen, sind Pioniere auf diesem Gebiet, die ihre ganze Kraft darauf verwenden müssen, unterrichtspraktisch zu handeln, ohne auf die unterstützende und absichernde Rahmung einer islamischen Religionsdidaktik zurückgreifen zu können.

Viele Anfangsschwierigkeiten sind überwunden und die Lehrkräfte können souverän auf Nachfragen der Eltern reagieren, die mitunter im islamischen Religionsunterricht eher die Vermittlung von Inhalten als die Reflexion über Inhalte erwarten.

In mehrtägigen Fortbildungskursen wurden und werden die Lehrkräfte fortgebildet. Viele der islamischen Religionslehrkräfte verfügen über eine islamisch- 
theologische Ausbildung im Herkunftsland und können gewinnbringend ihr fachliches Wissen in die Fortbildung einbringen.

Die am Schulversuch beteiligten Lehrkräfte sind im besonderen Maße gefordert:

Sie mussten und müssen sich didaktisch-methodisches Wissen und Können aneignen, das sich deutlich von der bisherigen Arbeit, die im herkunftssprachlichen Unterricht zu leisten ist, unterscheidet.

Sie haben sich in ein neues Unterrichtsfach eingearbeitet, und tragen erfolgreich dazu bei, eine Didaktik für ein Fach, das derzeit im Entstehen ist, mit zu entwickeln.

Sie müssen sich mit den verschiedenen muslimischen Gemeinden und Verbänden auseinandersetzen.

Sie tragen zur Transparenz des Schulbesuchs bei, indem sie Anfragen am Schulversuch Interessierter beantworten, Hospitationen zulassen und sich immer wieder für Interviews der öffentlichen Medien zur Verfügung stellen.

\section{Bisherige Bilanz des Schulversuchs}

Man sollte und darf den Schulversuch nicht überfrachten. „Islamischer Religionsunterricht“" ist sicherlich kein Allheilmittel für ungelöste interreligiöse Verständigungsschwierigkeiten und Integrationsprobleme. Aber es besteht die begründete Annahme, dass der „Islamische Religionsunterricht“ eine neue Phase des Zusammenlebens von Nicht-Muslimen und Muslimen in Niedersachsen eingeleitet hat. Das belegen die Ergebnisse der wissenschaftlichen Begleitung eindrucksvoll. Der Islamische Religionsunterricht trägt zudem dazu bei, dass das gesellschaftliche Miteinander von Menschen unterschiedlicher Religionen in diesem Land immer mehr zur Normalität wird, die von Offenheit und Toleranz geprägt ist.

Der Schulversuch ist nach dem, was an Evaluationsergebnissen vorliegt, erfolgreich. Rückmeldungen der Lehrkräfte, Schulleitungen und Eltern sowie die Ergebnisse der wissenschaftlichen Auswertung belegen, dass die von der niedersächsischen Landesregierung angestrebten integrativen Ziele auch tatsächlich erreicht werden:

Die Schülerinnen und Schüler nehmen sehr motiviert am Unterricht teil und erleben sich durch den islamischen Religionsunterricht als gleichgestellt mit den Schülerinnen und Schülern des christlichen Religionsunterrichts. Die Beteiligung seitens der muslimischen Schülerinnen und Schüler liegt in den einzelnen Jahrgängen oft bei nahezu $100 \%$.

Bei den Schülerinnen und Schülern hat im Laufe des Schulversuchs die Orientierung in Richtung Integration leicht zugenommen, und separatistische Neigungen haben deutlich abgenommen. Sowohl bei den Schülern als auch bei den Eltern ist die vorherrschende Orientierung die Integration. Bei den Schülerinnen und Schülern und bei deren Eltern besteht der 
Wunsch nach Teilhabe, Partizipation an der Mehrheitsgesellschaft, Kontakt und Freundschaften zu Deutschen, aber auch der Wunsch, die familialen Traditionen der eigenen Eltern beizubehalten

Der Schulversuch „Islamischer Religionsunterricht an den Grundschulen in Niedersachsen" hat bei allen Beteiligten den Schülerinnen und Schülern, den Eltern sowie den Lehrkräften über den gesamten Zeitraum von mehr als fünf Jahren hinweg eine unverändert hohe Akzeptanz.

Als nicht erwarteter Effekt hat sich gezeigt, dass der Unterricht zu einer generellen Steigerung der Sprachkompetenz der Schülerinnen und Schüler führt, da die Erprobung der deutschen Sprache, ohne in der empfundenen Konkurrenz zu den deutschen Mitschülerinnen und Mitschüler zu stehen, die Beherrschung abstrakter Begriffe zu begünstigen scheint.

Auch wenn die Eltern grundsätzlich mit dem „Islamischen Religionsunterricht" sehr zufrieden sind, so gibt es natürlich auch kritische Äußerungen seitens der Eltern. Einige Eltern fordern die Vermittlung von mehr Faktenwissen und eine traditionelle Wertevermittlung oder kritisieren Unterrichtsmethoden wie das Singen, Malen oder Tanzen. Für diese Eltern ist das Singen fröhlicher Lieder nicht in Einklang zu bringen mit dem ernsten Thema Religion. Das Darstellungsverbot des Propheten wird auf jede bildliche Darstellung im Unterricht überhaupt übertragen. Ein schüler- oder problemorientierter Unterricht, der zu unterschiedlichen Ergebnissen oder Bewertungen religiöser Inhalte führt, entspricht nicht den Erwartungen dieser Eltern. Den Lehrkräften scheint das konstruktive Gespräch mit den „kritischen“ Eltern zu gelingen, denn auch wenn die Erwartungen dieser Eltern nicht erfüllt werden oder wurden, so kommt es nicht zu Abmeldungen, wie die hohen Teilnahmequoten zeigen.

Zusammenfassend kann gesagt werden, dass gerade die Ergebnisse der wissenschaftlichen Begleitung zu dem Schulversuch „Islamischer Religionsunterricht“ als ein wichtiger Hinweis gedeutet werden können, dass die von der Niedersächsischen Landesregierung angestrebten integrativen Ziele auch tatsächlich erreicht werden. Der Schulversuch trägt zur Integration bei und stärkt die kulturelle Identität muslimischer Schülerinnen und Schüler. Annahmen, der „Islamische Religionsunterricht" könnte separative Haltungen oder die Ablehnung anderer Religionen stärken, können eindeutig zurückgewiesen werden. Muslimische Schülerinnen und Schüler möchten genauso offen und respektvoll mit anderen Religionen umgehen wie christliche bzw. nicht-konfessionelle Schüler und Schülerinnen.

\section{Einführung Islamischen Religionsunterrichts an öffent- lichen Schulen als ordentliches Unterrichtsfach}

Die Diskussion, islamischen Religionsunterricht von in Deutschland ausgebildeten Lehrkräften und unter deutscher Schulaufsicht im Sinne von Artikel 7 Abs. 3 GG 
zu erteilen, wird nicht nur auf Länderebene geführt, sondern auch auf Bundesebene. Im Rahmen der Deutschen Islamkonferenz (DIK) wurden die verfassungsrechtlich maßgeblichen Voraussetzungen für die Einführung islamischen Religionsunterrichts in Form einer Handreichung weiter konkretisiert. Dabei spielte die Behandlung der Frage, unter welchen Voraussetzungen der islamische Religionsunterricht eingeführt werden kann bzw. welche Voraussetzungen vorliegen müssen, damit die Einführung des Unterrichts verfassungskonform ist, eine besondere Rolle. Die Kultusministerkonferenz hat den Ländern empfohlen, auf Grund dieser verfassungsrechtlichen Rahmenbedingungen eines islamischen Religionsunterrichts zu prüfen, ob islamischer Religionsunterricht eingeführt werden könnte.

Als entscheidende Voraussetzung, die vorliegen muss, damit ein konfessioneller Religionsunterricht an den öffentlichen Schulen eingerichtet werden darf, wurde in der Handreichung formuliert, dass eine Religionsgemeinschaft vorhanden sein muss, die ihre Grundsätze für den Religionsunterricht definiert und Organe oder Ansprechpartner benennt, die diese Grundsätze dem Staat gegenüber zur Geltung bringt. Regelungen über den Islamischen Religionsunterricht könnten dann auch Gegenstand einer Vereinbarung zu unterscheiden von einem religionsverfassungsrechtlichen Vertrag zwischen der Religionsgemeinschaft und dem Land sein.

Da die Einführung von Religionsunterricht nicht von einem Körperschaftsstatus der jeweiligen Religionsgemeinschaft abhängig ist, kann auch mit einer Religionsgemeinschaft, die lediglich vereinsrechtlich organisiert ist, eine Vereinbarung über den islamischen Religionsunterricht geschlossen werden.

Um als Ansprechpartner für den Staat auftreten zu können, müssten die Verbände in Niedersachsen die genannten Voraussetzungen erfüllen, um dann gegenüber dem Land aktiv werden zu können.

\section{Ausblick}

Die Forderung des Runden Tischs, islamischen Religionsunterricht als ordentliches Unterrichtsfach einzuführen, wird von der niedersächsischen Landesregierung sehr ernst genommen. Für eine flächendeckende Einführung von islamischem Religionsunterricht als ordentlichem Unterrichtsfach sind allerdings nicht nur die verfassungsrechtlichen Probleme zu lösen, sondern auch die Probleme der Unterrichtsversorgung.

In den Schuljahrgängen eins bis elf werden in Niedersachsen ca. 50.000 Schülerinnen und Schüler islamischen Glaubens beschult. Um den Bedarf an universitär ausgebildeten Lehrkräften zukünftig abdecken zu können, wird seit dem Wintersemester 2007/2008 an der Universität Osnabrück der Masterstudiengang „Islamische Religionspädagogik“ angeboten. In diesem Ergänzungsstudiengang werden islamische Religionslehrer für Grund-, Haupt- und Realschulen ausgebildet. Mit den ersten Studienabsolventen der Universität Osnabrück ist erst ab dem Jahr 
$2011 \mathrm{zu}$ rechnen. Es wird also noch eine Weile dauern, bis es genügend universitär ausgebildete Lehrkräfte gibt. Bis dahin wird sich Niedersachsen der Herausforderung stellen müssen, wie die Unterrichtsversorgung im Schulversuch (oder später einmal Fach islamische Religion) gewährleistet werden kann.

Dem Schriftsteller Erich Kästner wird die Lebensphilosophie zugeschrieben: „Es gibt nichts Gutes, außer man tut es“. Genau dieser Philosophie ist die Niedersächsische Landesregierung gefolgt, indem sie trotz rechtlicher Schwierigkeiten und pädagogischer sowie organisatorischer Herausforderungen den Schulversuch umgesetzt hat.

\section{Literatur}

Bade, R./ Windolph, E.: "Islamischer Religionsunterricht” - ein niedersächsischer Schulversuch. Schulverwaltungsblatt für Niedersachsen, 12/2003, 389-391 2003

Ballasch, H.: Auf dem Weg zum Islamischen Religionsunterricht. Der Schulversuch in Niedersachsen. Schulverwaltung. Ausgabe Niedersachsen und Schleswig-Holstein 10/2005, 269-271 2005

Ballasch, H./ Haci-Halil U.: Schulversuch „Islamischer Religionsunterricht“" Schulverwaltung. Ausgabe Niedersachsen und Schleswig-Holstein 12/2008, 334-335 2008

Dahling-Sander, C./ Kraft, F. (Hrsg.): Islamischer Religionsunterricht - Wohin führt der Weg? Zwischenbilanz und Ausblick. Haus kirchlicher Dienste der Ev.-luth. Landeskirche Hannovers 2006

Mohr, I.: Zur Konstruktion von Unterrichtsgegenständen und Bildungszielen. In: Zeitschrift für die Religionslehre des Islam, 2/2008, 2-9 2008

Schmid, H.: Neue Phase des Zusammenlebens - Schritte auf dem Weg zum Islamischen Religionsunterricht. Herder Korrespondenz 59, 5/2005, 239-244 2005

Niedersächsisches Kultusministerium: Die niedersächsischen allgemein bildenden Schulen in Zahlen, Stand: Schuljahr 2007/2008, 382008

Heinrich de Wall/UAG der AG 2: Verfassungsrechtliche Rahmenbedingungen eines islamischen Religionsunterrichts. Deutsche Islamkonferenz (DIK): Zwischen-Resümee der Arbeitsgruppen und des Gesprächskreises. Vorlage für die 3. Plenarsitzung der DIK. 13. März 2008 Berlin, 19-27 2008 


\section{Kinan Darwisch: Islamischer Religionsunterricht in Deutschland und seine Entwicklung in Nieder- sachsen}

\section{Die Ausgangslage}

Cirka 3,5 Millionen Muslime leben in Deutschland, deren Integration in die überwiegend christlich geprägte demokratisch-säkulare Gesellschaftsordnung des Grundgesetzes problematischer erscheint, als dies bei anderen Migranten- und Religionsgruppen der Fall ist.

Aus diesem Grund stehen intensivierte Bemühungen zur Integration der Muslime seit der Jahrtausendwende -insbesondere nach den Anschlägen des 11. September 2001- vermehrt auf der politischen Agenda. Vor allem die jüngere muslimische Generation steht dabei im verstärkten Blickwinkel von Integrationsbemühungen des Staates und Institutionen der Mehrheitsgesellschaft, da diese unter anderem besonders gefährdet erscheint, von extremistischen Kreisen angeworben und für ihre Ziele instrumentalisiert zu werden.

Ferner leiden jugendliche muslimische Migranten erhöht unter Isolation in den Schulen sowie erheblichen Disparitäten in ihrem schulischen Erfolg (vgl. Gesemann 2006, S. 14ff.; Stanat 2006, S. 98ff.), was allerdings bei Betrachtung der lang- 
jährigen staatlichen Vernachlässigung der hierzulande lebenden muslimischen Migranten(kinder) nicht überrascht.

Als ein notwendiger Schritt zu einer besseren Integration der Muslime in die deutsche Gesellschaft wird vor allem die Einrichtung eines ordentlichen islamischen Religionsunterrichts in deutscher Sprache nach Artikel 7 III GG an öffentlichen deutschen Schulen gesehen. Ein deutschsprachiger öffentlicher islamischer Religionsunterricht soll dazu beitragen, dass muslimische Jugendliche zu mündigen Bürgern erzogen werden, die fähig sind, Tradition und Glauben kritisch zu hinterfragen, eine mündige Glaubensentscheidung zu fällen sowie zu einsichtigem und eigenverantwortlichem Handeln in einer demokratischen Gesellschaft bewogen werden (vgl. Ballasch in: Langenfeld/ Lipp/ Schneider 2005 (Hrsg.), S. 74; Kaddor/ Nieland 2008, S. 75). Desweiteren soll den Muslimen aber auch das Recht auf einen ordentlichen Religionsunterricht zugesprochen und ihre Gleichbehandlung vorangetrieben werden (vgl. Bade in: Langenfeld, Lipp/ Schneider 2005 (Hrsg.), S. 37ff.).

\section{Die rechtliche Lage und der fehlende muslimische An- sprechpartner}

Der ordentliche Religionsunterricht nach Art. 7 III GG stellt ein besonderes Recht der Religionsgemeinschaften dar, welches den Staat zur Veranstaltung und Finanzierung des Religionsunterrichts als ordentlichem und vor allem bekenntnisorientiertem Lehrfach im Rahmen des staatlichen Schulwesen und in Übereinstimmung mit den Grundsätzen der Religionsgemeinschaften verpflichtet (vgl. Von Campenhausen 2006, S. 190 ff.).

Nach Art. 7 I GG steht das gesamte Schulwesen unter staatlicher Aufsicht, was bedeutet, dass die Schulorganisation, der Lehrplan, die Schulaufsicht und die Lehrerausbildung dem Staat obliegen (vgl. ebenda, S. 190 ff.; Janke 2005, S. 39ff.). Ohne die staatliche Schulaufsicht zu verletzen, muss der ordentliche Religionsunterricht aber in Übereinstimmung mit den Religionsgemeinschaften erteilt werden. Die Religionsgemeinschaften haben unter anderem das Recht dem Lehrplan inhaltlich zuzustimmen sowie auch den Religionslehrern die Lehrbefugnis zu erteilen und gegebenenfalls wieder zu entziehen.

Das Recht auf die Einrichtung eines ordentlichen Religionsunterrichts gilt selbstverständlich für jede Religionsgemeinschaft. Doch gibt es bis heute in keinem Bundesland einen ordentlichen islamischen Religionsunterricht nach Art. 7 III GG, da die muslimischen Verbände bis heute in keinem Bundesland einen Ansprechpartner in Form einer Religionsgemeinschaft ${ }^{27}$ nach Art. 7 III GG gebil-

27 Nach Anschütz wird Religionsgemeinschaft als „eine die Angehörigen eines und desselben Glaubensbekenntnisses oder mehrerer verwandter Glaubensbekenntnisse (...) für ein Gebiet (ein Land, Teile eines Landes, mehrere Länder, das Reichsgebiet) zusammenfassender Verband 
det haben und damit in erster Linie den organisatorischen Anforderungen nicht genügen. Der Staat darf sich aufgrund seiner Neutralitätsverpflichtung nicht in die Bildung einer islamischen Religionsgemeinschaft einmischen oder gar einen der vielen muslimischen Verbände als Ansprechpartner im Sinne einer Religionsgemeinschaft auswählen.

\section{Der niedersächsische Schulversuch}

Um den religiösen Bedürfnissen der cirka 700.000 muslimischen Schülerinnen und Schüler in der Bundesrepublik jedoch bereits vor einer bzw. mehrer eventuellen sowie langwierigen islamischen Religionsgemeinschaftsgründungen in einem Mindestmaße gerecht zu werden und vor allem ihre Integration ins deutsche Schulund Gesellschaftssystem zu fördern, wurden in mehreren Bundesländern islamische Religionsunterrichts- und Islamkundeversuche in deutscher Sprache gestartet.

Das Bundesland Niedersachsen in dessen Schulen es cirka 50.000 Schülerinnen und Schüler muslimischen Glaubens gibt, nahm hierbei eine Vorreiterrolle ein. Mit Beginn des Schuljahres 2003/2004 startete an acht Grundschulen der Klassenstufen 1-4 der Schulversuch „Islamischer Religionsunterricht“, der dieses Jahr bereits auf 35 Schulen ausgeweitet werden soll.

Der Schulversuch soll als möglicher "Vorreiter" eines ordentlichen islamischen Religionsunterrichts im Sinne von Artikel 7 III GG in Niedersachsen fungieren, der nach dem Wunsch der beteiligten Parteien - insbesondere des Kultusministeriums - ursprünglich am Ende des fünfjährigen Schulversuchs, also im Jahr 2008 in Niedersachsen beginnen sollte (vgl. Erpenbeck/Windolph 2005, S. 48; Lögering 2006). Mittlerweile wird der Schulversuch jedoch vorerst im zweijahrestakt regelmäßig verlängert, da die Unrealisierbarkeit des Zustandebringens eines ordentlichen islamischen Religionsunterrichts bis zum Jahre 2008 von allen Beteiligten erkannt wurde.

Der Versuch wird zudem ausschließlich in den Klassen 1-4 durchgeführt, da dort kein Ersatzunterricht für das Fach Religion existiert und die muslimischen Schüler somit Freistunden hätten und dies aus der Sicht des Kultusministeriums mittlerweile nicht mehr mit dem Bildungsauftrag der Schule vereinbar wäre (vgl. Bade in: Langenfeld/ Lipp/ Schneider 2005 (Hrsg.), S. 39). Dies ist in den höheren Klassenstufen nicht mehr der Fall, da die muslimischen Schülerinnen und Schüler neuerdings gemäß $₫ 128$ NSCHG zur Teilnahme am Unterrichtsfach Werte und Normen verpflichtet sind, was zuvor ausschließlich für Religionsangehörige die Pflicht war, für die ein ordentlicher Religionsunterricht bereits eingerichtet wurde und die an diesem nicht teilnehmen wollten.

zu allseitiger Erfüllung der durch das gemeinsame Bekenntnis gestellten Aufgaben“ (Anschütz 1933, S. 633) definiert. 
Um jedoch einen größtmöglichen Konsens unter den Muslimen zu erzielen, lud das Kultusministerium zu einem Runden Tisch, die - wie es meint(e) - maßgeblichen Repräsentanten der organisierten Muslime ein, um mit diesen einen Schulversuch „Islamischer Religionsunterricht“ in den Klassenstufen 1-4 gemeinsam im Jahre 2003 zu starten. Der Runde Tisch soll hierbei eine beratende Funktion für die fehlende islamische Religionsgemeinschaft in Niedersachsen übernehmen, bis sich die Muslime zu einer solchen zusammengeschlossen haben und somit die Grundlagen für einen ordentlichen Religionsunterricht gemäß Art. 7 III GG vorliegen.

An den Runden Tisch eingeladen wurden von Beginn an:

- Schura - Niedersachsen, Landesverband der Muslime in Niedersachsen e.V.

- Türkisch-Islamische Union der Anstalt für Religion DITIB e.V. in Köln (Zentrale) sowie dessen Repräsentanz für Niedersachsen in Hannover

- Verband der Islamischen Kulturzentren e.V. in Köln und das zu ihm gehörende Islamische Kulturzentrum Hannover e.V., als maßgebliches Glied in Niedersachsen

- Islamische Gemeinde e.V. in Hannover (eine bedeutende bosniakische Gemeinde)

- Selman-Farsi Moschee (als bedeutende schiitische Moschee in Niedersachsen sowie Zweigstelle des Islamischen Zentrums Hamburg)

- ein Afghanischer Kulturverein (ebenfalls eine schiitische Gemeinde, deren Einfluss und Bedeutung in Niedersachsen jedoch in keiner Weise maßgeblich zu sein scheint)

- Vereinigung der Aleviten-Gemeinden e.V. in Köln (Zentrale) und Hildesheim

- Zentralrat der Muslime in Deutschland e.V.

Die IGMG (Islamische Gemeinschaft Milli Görüs) wurde kategorisch ausgeschlossen und die alevitische Seite mit einbezogen, die aber kurz vor Beginn des Schulversuchs den Runden Tisch verließ, da den Aleviten wahrscheinlich ihre Inhalte nicht ausreichend genug im Lehrplan berücksichtigt wurden schienen. Beides erregte den Protest der Sunniten und Schiiten am Runden Tisch, wie der Schura-Vorsitzende Altiner im April 2005 auf einer Fachtagung der Beauftragten der Bundesregierung für Migration, Flüchtlinge und Integration in Berlin hervorhob:

„Verfassungsrechtlich erscheint die Konstruktion eines Runden Tisches als Ersatz für die vom Grundgesetz geforderte „Religionsgemeinschaft nicht zulässig (...), da bereits mit der Zusammenstellung der Beteiligten (Aleviten-Bektaschiten) oder ihrem Ausschluss (z.B. IGMG) der Staat unzulässigerweise Einfluss auf die religiöse Inhaltsbestimmung und institutionelle Selbstbestimmung der Religionsgemeinschaft nimmt. Dies war offensichtlich von Anfang an seitens des Landes beabsichtigt. Aus Sicht des Landes ist nachvollziehbar, dass eine frisch gegründete 
islamische Religionsgemeinschaft so schnell nicht die Rolle des Kooperationspartners wahrnehmen soll.“ (Altiner 2005, S. 43).

Trotz alledem wurde in Übereinstimmung mit dem vom Kultusministerium zusammengestellten Runden Tisch der Lehrplan für den Schulversuch „Islamischer Religionsunterricht" in deutscher Sprache erarbeitet, der mit dem Bildungsauftrag der Schule gemäß $\ 2$ NSCHG übereinstimmt.

Der Religionsunterricht soll sich mit den existentiellen Fragen des Menschen, nach dem Woher und Wohin sowie dem Sinn und Ziel des menschlichen Seins befassen (vgl. Bade/ Windolph 2003, S. 389f.). Dabei steht im Islam der Glaube an Allah und die Beziehung zwischen ihm und dem Menschen sowie der gesamten Schöpfung, wie sie im Koran und der Prophetentradition überliefert wurde im Mittelpunkt. Weiterhin steht in den Rahmen-richtlinien des Kultusministeriums, dass der Unterricht die Aufgabe - an den Lernzielen der Schule orientiert - übernehmen soll, den Schülern zur Erkenntnis und Umsetzung zu verhelfen, dass:

„In einem kulturell vielfältigen, von unterschiedlichen Religionen und Weltanschauungen geprägten Umfeld (...) die Auseinandersetzung mit der eigenen Religion ein Beitrag zur Persönlicbkeitsbildung und religiösen Identitätsfindung [ist (Einfügung/ K.D.) ]. Sie ist zugleich Voraussetzung für das Verständnis und den Respekt gegenüber Angehörigen anderer Religionen und Weltanschaunngen. Toleran₹, gegenüber den unterschiedlichen Traditionen im Islam und verwandten Glaubensgemeinschaften wie auch gegenüber Angehörigen anderer Religions- und Weltanschaunngsgemeinschaften sollen im Schulversuch 'Islamischer Religionsunterricht' gefördert werden " Niedersächsisches Kultusministerium 2003, S. 5).

Didaktisch und methodisch orientiert sich der Lehrplan an einem zentralen Grundsatz katholischer Religionspädagogik, dem Korrelationsprinzip. Hierzu steht in den Rahmen-richtlinien:

„Aufgabe des Schulversuchs ist es, Glauben und Lehrpraxis mit der konkreten Lebenswirklichkeit und den Lebenserfahrungen der Schülerinnen und Schüler zusammenzuführen, so dass sie sich wechselseitig erschließen und erklären (Korrelationsprinzip).“ (ebenda, S.4)

In Beachtung dessen hat der Runde Tisch einen Konsens für den Lehrplan gefunden, dessen Text größtenteils auf dem Lehrplan des Zentralrats der Muslime in Deutschland e.V. für den Islamischen Religionsunterricht an Grundschulen basiert. Vorangegangen war eine umfassende Diskussion, in der die DITIB u.a. ein übersetztes Curriculum des türkischen Religionsministeriums vorlegt haben soll, dass sich jedoch „wegen darin enthaltener nationalistischer Elemente28 und einer Festschreibung der kemalistisch-laizistischen Staastdoktrin als inakzeptabel für

28 Wörtliches Zitat aus der Lehre für die 4. Klasse des zum Runden Tisch vorgelegten

Manuskriptes: „, Es sind sich darum zu bemühen, den SchülerInnen in der Liebe, Brüderschaft, Freundschaft und im Respekt zu erziehen und die Begriffen wie „Heimat, Nation, Flagge,

Gefallener und Frontkämpfer" zu begreifen“ 
deutsche Verhältnisse und muslimische Kinder“ (Altiner 2005, S. 42) erwiesen haben soll.

Der nun beschlossene Lehrplan hat folgende systematisch-thematische Inhaltsstruktur:

- Ich und meine Gemeinschaft

- Islamische Ethik

- Prophetengeschichten

- Prophet Mohammed

- Der Koran

- Grundlagen des Islam

Aus diesen Themenbereichen sind für die Grundschuljahrgänge 1-4 folgende verbindliche Themen konzipiert worden (vgl. Bade/ Windolph 2003, S. 389ff.):

- Gott (Allah)

- Gottes Schöpfung

- Der Koran

- Der Prophet Mohammed

- Die Propheten

- Beten

- Fasten

- Miteinander Leben

- Gemeinschaft der Muslime

- Begegnung mit anderen Religionen

Die fünf Säulen des Islam und die im Folgenden aufgelisteten Glaubenspfeiler des Islam werden vorausgesetzt:

- Der Glaube an den einen Gott

- Der Glaube an Gottes Engel

- Der Glaube an die göttlichen Schriften

- Der Glaube an die Propheten

- Der Glaube an das Leben nach dem Tod und

- Der Glaube an das Schicksal

Zum Unmut der Muslime wurde laut Schura ,ausdrücklich (...) vom Kultusministerium im Unterricht jegliche rituelle Praxis ausgeschlossen, also das Beten, Fasten oder Feste Feiern. Inwieweit ein konfessioneller Unterricht dies rechtfertigt - etwa in Relation zu Weihnachtsfeiern in der Schule -, wäre zu untersuchen. Zur muslimischen unverzichtbaren Glaubenspraxis gehören diese Elemente weiterhin und wir [ die Schura-Niedersachsen (Einfügung/ K.D.) ] meinen, sie können nicht einseitig aus dem konfessionellen Unterricht ausgeblendet werden." (Altiner 2005, S. 44)

Konkret soll der Runde Tisch laut Kultusministerium und der Ausländerbeauftragten des Landes eine beratende Beiratsfunktion für das Kultusministerium einnehmen sowie vor allem für die Akzeptanz auf muslimischer Seite Sorge tragen 
(vgl. Erpenbeck/ Windolph 2005, S. 51). Das Interesse der Landesregierung beim Schulversuch Islamischer Religionsunterricht sieht folgendermaßen aus:

„Die Landesregierung verfolgt mit dem Schulversuch das Ziel, den Schülerinnen und
Schülern muslimischen Glaubens ein Religionsunterrichtsangebot zu machen, das den
verfassungsmäßßgen und schulgesetzlichen Voraussetzungen entspricht. Sie anerkennt das
grundgesetzlich verbriefte Recht auf religiöse Identität und sieht in dem Angebot einen
Beitrag zur Integration der Schülerinnen und Schüler muslimischen Glaubens in die Ge-
sellschaft der Bundesrepublik." (Bade/ Windolph 2003, S. 390).

Somit ist das öffentlich geäußerte Interesse des Landes einerseits den Muslimen auf dem Weg zu ihrem Grundrecht auf einen ordentlichen Religionsunterricht im Rahmen des staatlich Möglichen zu helfen. Gleichzeitig soll aber auch die Integration der Muslime, die mehrheitlich Migranten sind, in die deutsche Gesellschaft gefördert und damit auch ein Beitrag zum Entgegenwirken der Bildung von Parallelgesellschaften geleistet werden.

Ein ganz anderes Problem stellte beim Schulversuch Islamischer Religionsunterricht die Adressatenfrage dar, da die Muslime und der Islam keine offizielle Mitgliedschaft kennen, wie dies in Europa bei den eingesessenen Religionsgemeinschaften der übliche Fall ist. Daher einigte man sich pragmatischerweise darauf, dass man eine Anmeldung der Kinder durch die Eltern zum Religionsunterricht (vorübergehend) als Mitgliedschaft zum Islam anerkennt, um dieses komplizierte Hindernis - vorerst für die Zeit des Schulversuchs - zu überwinden, was jedoch selbst einer islamischen Religionsgemeinschaft in Zukunft fast unmöglich zu werden scheint, eine andere als diese pragmatische Lösung durchzusetzen (vgl. Erpenbeck/ Windolph 2005, S. 50). Hierbei waren insbesondere die am Runden Tisch vertretenen Parteien hilfreich, indem sie den ihnen nahe stehenden Eltern eine Anmeldung ihrer Kinder zum Schulversuch empfohlen hatten. Dabei wird auch erkennbar, dass die am Runden Tisch vertretenen Parteien, einen nicht unerheblichen Einfluss auf die Teilnehmerzahl des Islamischen Religionsunterrichtsversuchs und somit letztlich seinem Erfolg haben und diesen auch bei der Wahrung ihrer Interessen am Runden Tisch mit einfließen lassen können.

Um die Qualifikation der Lehrerinnen und Lehrer zu gewähren sowie die Fundamente für eine islamische Religionsdidaktik zu setzen, wurde schließlich an der Universität Osnabrück ein islamischer Religionslehrerstudiengang eingerichtet.

Im Ergebnis kann gesagt werden, dass das Land Niedersachsen durchaus enorme Geldsummen sowie auch Arbeitsaufwand in diesen Schulversuch investiert hat, die sich letztlich auch insbesondere in Anbetracht der positiven Evaluationsergebnisse desselben, gelohnt zu haben scheinen.

Andererseits ist auch ersichtlich, dass insbesondere bei der Lehrplangestaltung sowie zudem auch bei der Lehrerausbildung und Lehrerauswahl Differenzen zwischen Mitgliedern des Runden Tisches und dem Kultusministerium vorzuliegen scheinen, deren Entwicklung abgewartet werden muss. 
Ein ordentlicher islamischer Religionsunterricht im Sinne von Art. 7 III GG ist in Niedersachsen gegenwärtig immer noch nicht einrichtbar, denn keiner der im Land vertretenen Muslime scheint bisher seriös den Status einer Religionsgemeinschaft einnehmen zu können, wenngleich es positive Entwicklungen diesbezüglich zu geben scheint und der gegenwärtige Zustand nicht von Dauer sein muss. Der niedersächsische Schulversuch stellt jedoch eine rechtlich mögliche Alternative dar, die potentiell durchaus geeignet zu sein scheint, alle Beteiligten weitgehend zufrieden zu stellen und somit den Integrations- und Gleichstellungsprozess der muslimischen Schüler und islamischen Organisationen in Niedersachsen, auch über den Rahmen von Art. 7 III GG, voranzubringen.

„Auf dem Weg zu Art. 7 III GG“ bedeutet jedoch, dass das Ziel vorliegen muss, insbesondere die beteiligten Muslime an die Position einer Religionsgemeinschaft im Sinne des Grundgesetzes heranzuführen.

Bei Betrachtung der aktuellen Lage des islamischen Religionsunterrichtsversuchs wird sichtbar, dass die Muslime am Runden Tisch zwar ein Mitspracherecht bei der inhaltlichen Gestaltung des Lehrplanrahmens hatten. Auf der anderen Seite haben sie jedoch keinerlei Einflussnahme auf die Lehrbefähigungserteilung (vgl. Missio Canonica und Vokation) und keine Kontrollmöglichkeit auf die Religionslehrer sowie Einsichtnahmemöglichkeit in den Religionsunterricht. Dies stellt gegenüber dem ordentlichen Religionsunterricht ein Defizit dar und es ist zu erwarten, dass die Muslime des Runden Tisches, insbesondere die DITIB und die Schura, dies nicht als Dauerzustand akzeptieren werden. Aus diesen Gründen ist nach Lösungsmöglichkeiten auf dem Weg zu Art. 7 III GG zu suchen, die dieses Konflikt-potential eindämmen.

Das größte Interesse des Staates scheint hierbei berechtigterweise die Wahrung der Verfassungskonformität eines islamischen Religionsunterrichts zu sein, der weder die Grundrechte (v.a. die Glaubensfreiheit und Gleichberechtigung der Geschlechter), beziehungsweise das Werte- und Rechtssystem des Grundgesetzes in Frage stellt. Den Muslimen jedoch wiederum liegt vor allem die Authentizität und Qualität der Verkündung der islamischen Lehre am Herzen, um den Kindern auch in der Schule zu helfen, eine islamische Identität, neben den beiden anderen islamischen Erziehungspfeilern Familie und Koranschule (Gemeinde), aufzubauen (vgl. Altiner 2005, S. 46f.).

Aus diesen Gründen liegt auf der Hand, dass muslimische Religionslehrerinnen und -lehrern einerseits nicht verfassungsfeindliches Gedankengut verkünden oder dazu von muslimischer Verbandsseite aufgefordert werden dürfen. Auf der anderen Seite muss es sich bei den Religionslehrerinnen und -lehrern jedoch auch um bekennende Muslime, sunnitischer oder schiitischer Konfession handeln, die sich zum islamischen Glauben bekennen und diesen auch adäquat und überzeugt verkünden.

Da die Muslime jedoch auch in Niedersachsen noch nicht die organisatorischen Voraussetzungen erfüllt haben kann der Staat diesen auch nicht die Lehrbe- 
fugniserteilungsmöglichkeit für Lehrkräfte zukommen lassen, wie dies bei den beiden großen Kirchen geschehen ist.

$\mathrm{Zu}$ groß wäre die Gefahr eines eventuellen Missbrauchs und Missverständnissen über die Befugnisse von muslimischer Seite, bei bisher nicht geklärten und partiell noch nicht angesprochenen Konfliktpotentialen, wie beispielsweise der Kleidung der Lehrerinnen und Lehrer im Religionsunterricht (oder evtl. auch außerhalb dessen), Esssitten von Lehrerinnen und Lehrern und anderen Punkten ihres Lebensstils, die bei Betrachtung der islamischen Religionslehre und Diskussionen innerhalb der muslimischen Community in diesem Kontext durchaus aufkommen könnten, jedoch die Freiheitsrechte der Lehrerinnen und Lehrer verletzen würden, wenn man in ihre Grundrechte einzugreifen versuchte und ihnen möglicherweise noch ihre Lebensführung vorschreiben wollte.

Aus diesem Grund wäre hierbei beispielsweise eine Lösungsmöglichkeit, dass die Muslime am Runden Tisch bei Zweifeln über die Konfession eines Religionslehrers, der im niedersächsischen Landesschulversuch aufgrund der aktuellen $\mathrm{Zu}$ sammenstellung des Runden Tisches verständlicherweise nur ein Sunnit oder Schiit sein sollte, die Möglichkeit eingeräumt bekommen, diese an das Kultusministerium weiterzuleiten und dieses weitere Schritte vornimmt, auch mit der eventuellen Folge der Enthebung dieser Person aus ihrer Funktion als Religionslehrer. Eine Lehrbefähigungserteilungsermächtigung ist ihnen jedoch (noch) nicht zu erteilen, bis die punktuell aufgeführten Konfliktpunkte geregelt sind. Hierbei allerdings wäre es seitens des Kultusministeriums ratsam, mit der Erarbeitung eines Kataloges über mögliche Wünsche und Vorstellungen der am Runden Tisch vertretenen Muslime zu beginnen, der auch später im Rahmen eines ordentlichen islamischen Religionsunterrichts als Grundlage für eventuelle Lehrbefähigungsentziehungen oder Verwarnungen von Lehrerinnen und Lehrern, Geltung haben könnte.

Alles zusammengefasst betrachtet liegt hierin ein sehr langer, komplex auszugestaltender, kosten- und nervenintensiver Prozess, in dem letztlich auch die inhaltlichen Konflikte eines islamischen Religionsunterrichts praktisch, aber auch der Umgang mit solchen Konflikten seitens der muslimischen Schülerschaft in der hiesigen multikulturellen und pluralistischen Gesellschaft gelöst werden könnten und daher durchaus viel versprechend ist. Eine Stelle hierfür müsste im Kultusministerium jedoch scheinbar noch eingerichtet werden, um einen pädagogischjuristisch, als auch islamisch ausreichend kompetenten Ansprechpartner für die Muslime zur Verfügung zu stellen.

Bei der Einsichtnahmemöglichkeit in den islamischen Religionsunterricht tritt das gleiche Problem wie bei der Lehrbefähigungsermächtigung auf. Den organisierten Muslimen kann auch hier noch keine Einsicht in den Unterricht erteilt werden, wenn sie selbst noch nicht ihre Defizite auf dem Weg zur Bildung einer islamischen Religionsgemeinschaft behoben haben. Auch hierbei wäre eine Lösungsmöglichkeit, dass die Muslime gemeinsam mit dem Kultusministerium Krite- 
rien für diese absprechen und aufstellen. Allerdings kann (noch) nicht dazu geraten werden, den muslimischen Partnern (des Runden Tisches) eine Kontrolleinsichtnahme in den Unterricht zu gewähren, durchaus jedoch im Rahmen des angesprochenen Entwicklungsprozesses in Aussicht gestellt werden. Dies wäre letztlich auch eine Hilfe für die Muslime bei der Behebung eigener organisatorischer und juristischer Probleme auf dem Weg zu einer staatlich anerkannten Religionsgemeinschaft, bei der sie durchaus äußere Hilfe benötigen zu scheinen.

Trotz der aufgeführten Konfliktpunkte und Schwierigkeiten scheint das Land Niedersachsen einen langfristig viel versprechenden Weg eingeschritten zu haben. Das Land ist den Muslimen entgegengekommen, denn es ist nicht die Pflicht des Landes einen solchen Islamischen Religionsunterrichtsversuch durchzuführen. Da jedoch der Staat insbesondere auch aus integrationspolitischen Gründen an einem islamischen Religionsunterricht interessiert ist, stellt der Schulversuch „Islamischer Religionsunterricht" in Niedersachsen eine sinnvolle Alternative zu Art. 7 III GG dar. Dieser Versuch bietet trotz einer fehlenden islamischen Religionsgemeinschaft einen islamischen Religionsunterricht für muslimische Schülerinnen und Schüler in Zusammenarbeit mit einem Großteil der in Niedersachsen organisierten Muslime an, der den muslimischen Schülerinnen und Schülern eine islamische Identitätsbildung in Einklang mit den verfassungsrechtlichen Werten des Grundgesetzes ermöglichen kann. Hierin liegt großes Potential, Integrationsprobleme muslimischer Schülerinnen und Schülern in der Schule und späterer erwachsener muslimischer Bürger in der Gesellschaft zu beheben. Auf der anderen Seite scheint den organisierten Muslimen des Runden Tisches auf dem Prozess der Bildung und Entwicklung einer islamischen Religionsgemeinschaft durchaus geholfen werden zu können, indem sie schon jetzt mit partiellen Aufgaben einer solchen vertraut gemacht und ihnen "step by step" mehr Gestaltungsmöglichkeiten und Mitspracherechte in die Hände gegeben werden.

Aus diesen Gründen muss der Staat darauf achten die Muslime auch in Zukunft wachsend in das Geschehen mit einzubinden, auf der anderen Seite jedoch wachsam ihren Prozess zur Bildung einer Religionsgemeinschaft beobachten.

Die organisierte muslimische Seite in Niedersachsen, vor allem die Ditib und Schura kommen jedoch langsam in die Bringschuld organisatorische sowie auch inhaltliche Fortschritte auf dem Weg zu einer Religionsgemeinschaft im Sinne von Artikel 7 III GG vorzuweisen. Vor allem die Schura erweckt den Eindruck, dass dort eine gewisse Stagnation eingetroffen zu sein scheint. Dies wäre bedauerlich, denn langfristig könnte sie durchaus das Potential haben auf Landesebene einen Religionsgemeinschaftsstatus einzunehmen.

Trotz alledem sollte deutlich geworden sein, dass alle Seiten mit diesem Schulversuch nur gewinnen können und er ein richtiger und wichtiger sowie vor allem von der Landesregierung mutiger und progressiver Schritt in Richtung Integration der Muslime in Niedersachsen ist. 


\section{Literatur}

Altiner, A.: Erfahrungen in der Kooperation am Beispiel des islamischen Religionsunterrichts aus Sicht des Landesverbandes der Muslime in Niedersachsen. In: Beauftragte der Bundesregierung für Migration, Flüchtlinge und Integration (Hrsg.): Islam einbürgern - Auf dem Weg zur Anerkennung muslimischer Vertretungen in Deutschland. Dokumentation der Fachtagung der Beauftragten der Bundesregierung für Migration, Flüchtlinge und Integration 25. April 2005. Berlin 2005, S. 42-47.

Anschütz, G.: Die Verfassung des Deutschen Reichs vom 11. August 1919. Ein Kommentar Für Wissenschaft und Praxis. Bad Homburg 1933.

Bade, R./ Windolph, E.: „Islamischer Religionsunterricht“ - ein niedersächsischer Schulversuch. In: Schulverwaltungsblatt für Niedersachsen, 55 (2003) 12, S. 389-391.

Bade, R.: Anmerkungen zur aktuellen Situation des Religionsunterrichts, in: Langenfeld, C./ Lipp, V./ Schneider, I. (Hrsg.): Islamische Religionsgemeinschaften und islamischer Religionsunterricht: Probleme und Perspektiven. Göttingen 2005, S. 37-43.

Ballasch, H.: „Islamischer Religionsunterricht“ - Praxisbericht zum Schulversuch in Niedersachsen, in: Langenfeld, C./ Lipp, V./ Schneider, I. (Hrsg.): Islamische Religionsgemeinschaften und islamischer Religionsunterricht: Probleme und Perspektiven. Göttingen 2005, S. 73-78.

Beauftragte der Bundesregierung für Migration, Flüchtlinge und Integration (Hrsg.): Islam einbürgern - Auf dem Weg zur Anerkennung muslimischer Vertretungen in Deutschland. Dokumentation der Fachtagung der Beauftragten der Bundesregierung für Migration, Flüchtlinge und Integration 25. April 2005. Berlin 2005.

Erpenbeck, G./ Windolph, E.: Erfahrungen in der Kooperation am Beispiel des islamischen Religionsunterrichts aus Sicht der Ausländerbeauftragten und der Landesregierung Niedersachsen. In: Beauftragte der Bundesregierung für Migration, Flüchtlinge und Integration (Hrsg.): Islam einbürgern - Auf dem Weg zur Anerkennung muslimischer Vertretungen in Deutschland. Dokumentation der Fachtagung der Beauftragten der Bundes-regierung für Migration, Flüchtlinge und Integration 25. April 2005. Berlin 2005, S. 48-53.

Gesemann, F.: Die Integration junger Muslime in Deutschland. Berlin 2006.

Janke, K.: Institutionalisierter Islam an staatlichen Hochschulen. Verfassungsfragen islamischer Lehrstühle und Fakultäten. Frankfurt am Main 2005.

Kaddor, L./ Nieland, J.: Herausforderungen und Chancen in Bildungseinrichtungen. Grundinformationen zum Islam und Anregungen zum Umgang mit muslimischen Kindern, Jugendlichen und ihren Eltern. Siegburg 2008. 
Langenfeld, C./ Lipp, V./ Schneider, I. (Hrsg.): Islamische Religionsgemeinschaften und islamischer Religionsunterricht: Probleme und Perspektiven. Göttingen 2005.

Lögering, A.: „Islamischer Religionsunterricht“ beginnt an niedersächsischen Grundschulen. Verfügbar unter: http://www.bistumosnabrueck.de/downloads/islam_ru.pdf\#search=\%221\% C3\%B6gering\%20rahmenrichtlinien\%20Schulversuch\%20islamischer\%20Rel igionsunterricht $\% 20$ niedersachsen $\% 22$. [Zugriffsdatum: 27.09.2006]

Niedersächsisches Kultusministerium: Rahmenrichtlinien für den Schulversuch „Islamischer Religionsunterricht“. Stand 27. Mai 2003

Stanat, P.: Disparitäten im schulischen Erfolg: Forschungsstand zur Rolle des Migrations-hintergrunds, in: Unterrichtswissenschaft. Zeitschrift für Lernforschung 2/2006, S. 98-123.

Von Campenhausen, A.: Staatskirchenrecht. Ein Studienbuch. München 2006. 


\section{Haci-Halil Uslucan: Islamischer Religionsunterricht in der Grundschule: Ein Modellversuch zur Nachahmung?}

\section{Religion und Integration}

Welche Funktion hat eigentlich Religion bzw. Religiosität noch in einer modernen Lebenswelt? Ragt sie nicht heraus wie ein archaischer Pfeil aus längst vergangenen Zeiten, in denen es vielleicht noch üblich war, transzendentale Erklärungen und transzendentalen Schutz für ganz irdische Probleme einzufordern? Ist sie nicht ein Relikt aus der Vorphase der „Entzauberung der Welt“? Kurz: Woher beziehen religiöse Lehren heute noch ihre Legitimität?

Neuere Untersuchungen zur Wertebindung zeigen eindringlich, dass Religiosität alles andere als ein obsoletes, vernachlässigbares Phänomen moderner Gesellschaften darstellt; bspw. bezeichneten sich in einer Studie rund 2/3 der befragten EU Bürger als religiös; in den USA waren es dagegen sogar rund 90\% (Bucher, 2005). Obwohl viele Religionen sich in der Moderne mit einem erhöhten Druck ihres Legitimationsnachweises konfrontiert sehen, scheinen Religionen auch in der Moderne sich einfach nicht wegdiskutieren zu lassen. 
Unbestritten ist eine wichtige Funktion religiöser Lehren u. a. in der Sinnstiftung menschlichen Handelns zu sehen; in der Fähigkeit, Vorgänge zu deuten, die sonst sinnlos blieben und die erfahrene Wirklichkeit verstehbar zu strukturieren. Darüber hinaus haben Religionen zugleich auch die Funktion, den Einzelnen in die Gesellschaft zu integrieren. In der Erziehung bspw. kann die Funktion religiöser Vorstellungen generell darin gesehen werden, bei dem Gläubigen bestimmte Handlungsdispositionen und Erwartungen zu wecken, die seine Wahrnehmung der Wirklichkeit, seine Alltagsroutinen und sein Denken lenken, aber auch Stimmungen und Motivationen wachrufen.

Aus einer kulturanthropologischen Sicht wird Religion vielfach als eine Form der Kontingenzbewältigung verstanden, die die erfahrene Zerbrechlichkeit des Alltags bearbeiten hilft. So kann Religiosität insbesondere dort, „wo alles trostlos erscheint“", Handlungsmöglichkeiten eröffnen und Trost spenden, wo keine Handlungsmöglichkeiten mehr bestehen (vgl. Tan, 1999, S. 50).

Auch ein Blick auf die philosophische Anthropologie hilft, diese Sicht zu präzisieren: Primär wird der Mensch hier als ein instinktarmes, aber dafür ein weltoffenes Wesen betrachtet. Gerade durch das Fehlen einer von Instinkten gelenkten Leitung, durch diesen konstitutiven Mangel an Handlungs- und Orientierungssicherheit während seiner Frühphase, braucht der Mensch Erziehung, um handlungsfähig in der Welt zu sein und sich darin zu orientieren; und eine dieser Orientierungssysteme, die den Menschen aus seiner unmittelbare Handlungssphäre hinaus weisend in den Kontext von Mensch und Kosmos stellt, bildet die Religion.

Aus kulturpsychologischer Perspektive wirken religiöse Überzeugungen als Symbolsysteme, die menschliches Handeln organisieren. Diese sind dynamisch, sind einem sozialem Wandel unterworfen, und erweisen sich erst durch ihre Bedeutung im Kontext von Gebrauch und Handeln als lebendig. Deshalb müssen auch einmal etablierte religiöse Sinnsysteme in der Erziehung jeder neuen Generation fortwährend aktualisiert werden. Mit Blick auf kindliche Entwicklungen ist festzuhalten, dass die Entwicklung religiöser Überzeugungen und Wissensinhalte die Produkte einer Kind-Umwelt-Interaktion sind. Und fokussiert auf die Erziehung lässt sich formulieren: Innerhalb religiöser Symbolsysteme geht es nicht stets um rationales Verstehen, sondern vielfach eher um die Herstellung einer positiven affektiven und spirituellen Basis zum Glauben. Als exemplarisch ließe sich hier im Islam das Rezitieren oder das Zuhören von Koranversen nennen, die von vielen nichtarabischen Muslimen inhaltlich nicht verstanden werden. Jedoch werden damit auch verschiedene Ziele angesteuert: Primär soll dadurch ein positiver, wohlwollender „Habitus“ gegenüber der Religion erreicht, eine Implementierung eines affektiven Ankers erwirkt werden, an den im Laufe der Sozialisation bestimmte Inhalte angeknüpft werden können. Ähnliche Formen der Hinführung zur Religion lassen sich natürlich auch in anderen Religionen zeigen. Möglicherweise ist diese frühe Etablierung einer affektiven Basis - und nicht so sehr des 
rationalen Verstehens - verantwortlich für eine feste Verankerung des religiösen Systems in der Person.

Mit Blick auf das Leben in modernen Gesellschaften ist festzuhalten, dass von gegenwärtigen Ambivalenz- und Desintegrationserfahrungen Migranten deutlich stärker betroffen sind. Sehr viele Migranten aus ursprünglich ländlichen Regionen erleben die deutsche Gesellschaft als ungeordnet, sie erkennen keine dominanten kulturellen Werte. Das soziale Leben wirkt für sie diffus und undurchsichtig. In diesem Kontext hat Religion bspw. für die Eltern im Erziehungsalltag eine große Ordnungsfunktion. Das heißt: Die Orientierung am Islam hilft, die in der Moderne - übrigens auch für deutsche Eltern - immer schwerer gewordene Frage nach angemessenen Erziehungsinhalten zu vermeiden bzw. zu umgehen. Positiv formuliert: Der Islam gibt klare Regeln und eine Orientierung vor und reduziert dadurch Komplexität.

Die Schutz und Orientierungsfunktion hatte der Islam insbesondere im Leben vieler türkischer Migranten der ersten Generation, die zunächst in ghettoartigen Kontexten lebten und durch Errichtung von Gebetsstätten sowohl Orte sozialen Kontaktes als auch spiritueller Seelsorge um die Religion herum schufen (Akbulut, 2003). Gerade die Pluralisierung der Lebenswelt kann für Menschen, die aus einer eher homogenen kulturellen Umwelten stammen, wo Gewissheiten des Alltags stärker verbürgt sind, zum Problem werden, sodass eine Besinnung, Rückkehr oder Zuflucht zur Religion als eine Reduktion von Komplexität empfunden wird und somit kognitiv entspannend wirkt (Luhmann, 1982). Erlebte Zufälligkeiten bekommen dann einen Sinn und werden in eine Ordnung eingerahmt (z.B. als Wille Allahs, als göttliche Vorbestimmung, als Kismet etc. gedeutet). Zudem schützt Ordnung letztlich vor anomischen, als regel- und strukturlos empfundenen, Verunsicherungen in Zeiten sozialen Wandels. Gleichwohl schaffen aber religiöse Orientierungen auch neue Ambivalenzen (z. B. wie eine religiöse Sinnstiftung in der säkularen Moderne noch möglich ist).

Gerade in der Diaspora erlangt der Islam möglicherweise gegenüber migrationsbedingt erlittenen Kränkungen eine Überhöhung und wird stärker identitätsrelevant als in der Herkunftskultur: Viele Migranten aus islamischen Ländern erleben in der Fremde die Religion bewusster. Religiosität erscheint, auch wenn sie aus einer Situation der ,geistigen Obdachlosigkeit" heraus erfolgt, als eine frei gewählte Option, während sie in den Herkunftsländern vielfach eine vorgegebene und von der sozialen Mitwelt getragene Orientierung darstellt. Diese Formen der bewussten Rückwendung sind jedoch nicht islam-, sondern vielmehr migrationsspezifisch, d.h. sie betreffen auch andere Migranten in anderen Teilen der Welt. Gerade mit dem Familiennachzug stellt sich für viele (muslimische) Migranten die Frage der Weitergabe der eigenen Tradition und Religion an die nachwachsende Generation, dies um so mehr, je stärker sich die Familien in der Fremde bedroht erleben, Rückzugstendenzen in eigene kulturelle Muster zeigen und das Abgrenzungsbedürfnis stärker erleben. Durch die stärker religiöse Orientierung 
der Erziehung werden zugleich auch eine Rückbindung und ein Verstehen der Lebenswelt der Eltern gewährleistet und eine Möglichkeit der intellektuellen Auseinandersetzung mit der eigenen Tradition geschaffen.

Während eine religiöse Sozialisation in den islamischen Ländern vielfach vom Kontext unterstützt sowie z. T. unreflektiert als eine Alltagsgewissheit übernommen wird und durch die umgebende Gesellschaft eine Koedukation erfolgt, ist davon auszugehen, dass in der Migrationssituation - dort, wo der bestätigende und unterstützende Kontext entfällt - eine gezielte islamische Erziehung erfolgt. So beobachtet Schiffauer (1991) in der Migration eine „Islamisierung des Selbst“, die mit einer Individualisierung einhergeht. In der Migration ist die Gemeinde nicht vorgegeben, sondern sie kann gewählt werden; dadurch ist es nicht nötig, durch äußere Kennzeichen und Rituale die Zugehörigkeit zu bekunden. Die Suche nach (religiöser) Wahrheit wird intensiviert und die Zugehörigkeit zum Islam stärker spiritualisiert.

Was die Frage des Integrationspotentials des Islam im Allgemeinen betrifft, so wurde diese Problemwahrnehmung lange von einer eher abträglichen Haltung beherrscht; insbesondere wurden Koranschulen als Orte der Indoktrination mit eindeutigen Segregationstendenzen betrachtet, in denen muslimische Kinder von Einflüssen der deutschen Gesellschaft und demokratischer Prinzipien ferngehalten werden würden (vgl. Sackmann, 2001). Jedoch ist diese heikle Frage nicht so eindeutig zu beantworten: Denn in der Migrationssituation können Moscheen sowohl integrativ als auch ausgrenzend wirken, je nachdem mit welcher Ausprägung der Islam dort gelebt und gepredigt wird. Zumindest ist festzuhalten, dass Moscheen als „Umschlagplatz des Alltagswissens“ Muslimen helfen, sich in der Fremde zurechtzufinden und elementare Regeln und Fertigkeiten des sozialen Lebens, wie etwa Ausfüllen eines Antrages, zu erlernen etc. Ferner machen Muslime dort Erfahrungen der Zugehörigkeit zur Umma (Gemeinschaft), die insbesondere dann von Bedeutung ist, wenn der soziale Alltag ihnen häufig Ausgrenzungserfahrungen bereitet bzw. Unterlegenheitsgefühle produziert. In der Moschee wird dagegen die eigene Identität unter seinesgleichen bewahrt und bestärkt; in diesem Sinne kann eine praktizierte Religiosität auch als Schutz vor einer Identitätskrise gedeutet werden. In der Untersuchung von Heitmeyer, Müller und Schröder (1997) berichteten bspw. zwischen 34\% und 39\% der befragten Jugendlichen von Diskriminierungserfahrungen in Deutschland; rund zwei Drittel der Befragten bekundeten, der Islam bzw. die Zugehörigkeit zum muslimischen Religionskreis stärke ihr Selbstvertrauen. Auch Studien zur Konversionserfahrungen zeigen, dass sich eine neue Religiosität vielfach als eine Bewältigungsstrategie bzw. als Überwindung einer Identitätskrise verstehen lässt (Bucher, 2005).

Antiintegrative Folgen sind jedoch dann zu erwarten, wenn Moscheen dazu missbraucht werden, islamzentrierte Überlegenheitsgefühle zu produzieren, Differenzen zu verstärken oder bewusst zur Kontaktmeidung mit „Heiden“ oder Christen aufrufen und eine Selbstgenügsamkeit der Muslime suggerieren. Ein so ver- 
standener, fundamentalistischer Islam wird sich als dysfunktional erweisen, da er den notwendigen Dialog mit der Mehrheitsgesellschaft hemmt und das Zusammenleben erschwert. Denn die Überzeugung, dass die eigene Gruppe im Besitz der unverrückbaren Wahrheit ist, also ein exklusiver Heilsanspruch vertreten wird, gestaltet die Ausgangssituation für einen Dialog denkbar ungünstig. Jedoch scheint bei genauer Betrachtung gläubigen Muslimen nicht so sehr das Christentum als ernsthafte Konkurrenz zu fungieren, sondern vielmehr die Verlockungen der säkularen Welt (Zirker, 1993).

\section{Islam in Deutschland}

Nach den Erfahrungen des 11. Septembers 2001 ist recht voreilig die Integration islamischer Zuwanderer in Deutschland für gescheitert erklärt worden, obwohl die empirische Frage, wie die tatsächlichen Integrationschancen und der Integrationsstand muslimischer Migranten einzuschätzen ist, genauso wenig gelöst ist wie die nach eindeutigen Konzepten.

In Deutschland leben etwa 3.200.000 bis 3.500.000 Menschen islamischen Glaubens. Mit einem Bevölkerungsanteil von etwa 4\% ist der Islam in Deutschland neben dem Christentum die zweitstärkste Religion (Baumann, 2001). Darüber hinaus verzeichnet der Islam innerhalb der Weltreligionen die höchsten Zuwachsraten. Der Islam ist daher nicht mehr ein fremdes Element, sondern ein die europäische bzw. deutsche Kultur mit prägendes Phänomen ${ }^{29}$. Multikulturelle und multireligiöse Gesellschaft ist bereits eine soziale Tatsache; der Dialog mit muslimischen Migranten erweist sich als drängender denn je. Rund 750.000 Schülerinnen und Schüler muslimischen Glaubens besuchen deutsche Schulen (Müller, 2001). Bundesweit sind somit ca. 6\% aller Schülerinnen und Schüler islamischen Glaubens. Die Zugehörigkeit zum Islam ist ein Bestandteil der kulturellen Identität.

Der größte Teil der in Deutschland lebenden Muslime sind türkische Sunniten; innerhalb der islamischen Rechtsschulen folgen sie der hanefitischen Tradition. Kurdische Muslime aus der Türkei dagegen sind in ihrer religiösen Praxis stärker von der schafiitischen Tradition geprägt; Aleviten aus der Türkei wiederum haben

29 Trotz einiger gegenwärtiger gemeinsamer Entwicklungstrends dürfen jedoch Probleme im Zusammenleben der jeweiligen europäisch-christlichen Mehrheitsbevölkerung und der islamischen Minderheit im Lande nicht auf eine strukturelle, auf den Islam bezogene Konfliktdimension, zurückgeführt werden. So hat das Verhältnis von Muslimen in Frankreich, die vornehmlich aus Nordafrika kommen und in England, die vielfach aus Indien und Pakistan stammen, eine aufgrund der Kolonialisierungsgeschichte zum Herkunftsland eine zusätzliche historische, von Erniedrigung und Ausbeutung gekennzeichnete Konfliktdimension, die bspw. im Falle türkischer Muslime in Deutschland in dieser Form nicht vorliegt. Ganz im Gegenteil: historisch herrschten zwischen Deutschland und der Türkei bzw. dem osmanischen Reich stets eher gute Beziehungen vor. 
andere religiöse Praxen. Deshalb ist eine Homogenisierung in der Form, dass alle Muslime gleich seien, problematisch.

Wenn im Folgenden von Muslimen (synonym mit Moslems) bzw. islamischen Gesellschaften die Rede ist, so ist stets dabei im Auge zu behalten, dass die Dichotomie zwischen islamisch vs. nicht-islamisch in dieser Form nicht zulässig ist. Vielmehr besteht ein fließendes Kontinuum der Frömmigkeit von traditionsgebundener, unreflektierter Religiosität, bewusster Hinwendung zum Islam, aufgeweichter, kompromissbereiter Haltung zum Islam, überzeugten Säkularisten bis hin zur bewussten Ablehnung und atheistischen Haltung (Büttner, 2000).

\section{Ergebnisse zur Akkulturationsorientierungen der Schü- lerinnen und Schüler}

Schule ist der exemplarische Ort ist, an dem sich deutsche Schülerinnen und Schüler und Schülerinnen und Schüler nichtdeutscher Herkunft begegnen und Schule ist der Ort, in dem das Zusammenleben am besten eingeübt werden kann. Daher stellt sie par excellence das Feld gelingender oder misslingender Integration dar. Besonders in den letzten Jahren ist Schule jedoch weitestgehend als Schauplatz des Zusammenpralls eines säkularen und religiösen Weltbildes wahrgenommen worden. Neben den Debatten um einen islamischen Religionsunterricht waren in jüngster Vergangenheit die Versuche muslimischer Eltern, ihre Kinder bzw. ihre Töchter vom Sport- und Schwimmunterricht, vom Sexualkundeunterricht zu befreien oder auch die Teilnahme ihrer Kinder an Wandertagen und Klassenfahrten zu unterbinden, Gegenstand der Diskussion, was in Folge die Integrationschancen muslimischer Kinder nicht unwesentlich beeinflusst (Meyer \& Weil, 2000.)

Dabei kann die Warnung vor einer allzu schnellen Verallgemeinerung nicht stark genug unterstrichen werden: denn inwieweit die islamischen Erziehungsvorgaben und -muster tatsächlich für die jeweiligen muslimischen Familien Gültigkeit haben, ist vielfach von Merkmalen wie etwa ländliche oder städtische Herkunft, soziale Schicht und Bildungsgrad, Religiosität der eigenen Eltern abhängig. Dabei werden mitunter gewöhnliche Regeln der Alltagsgestaltung vorindustrieller Kulturen von den Beteiligten religiös bzw. islamisch begründet und ebenso kaum reflektiert, dass es zum Teil auch innerhalb des Islam gravierende Unterschiede in den verschiedenen Ausrichtungen vorherrschen (vgl. Stöbe, 1998).

\subsection{Evaluation des schulischen Modellversuchs in Niedersachsen}

Im Folgenden soll in Auszügen über die ersten Ergebnisse des Schulversuchs „Islamischer Religionsunterricht“ an den Grundschulen in Niedersachsen berichtet werden, der sowohl Fragen der Integrationsorientierungen als auch die Akzeptanz dieses modellhaften Unterrichts seitens der Schüler thematisiert. Eine aus- 
führliche Dokumentation der Ergebnisse liegt dem Niedersächsischem Kultusministerium vor.

In einem in der empirisch-wissenschaftlichen Forschung üblichen Pretest (Vorversuch) wurde der für die Schülerbefragung der Jahrgangsstufen 3 und 4 erstellte Fragebogen vor dem ersten Einsatz erprobt und dem Wissen und Können der Schülerinnen und Schüler angepasst. Die Erfahrungen der weiteren Befragungen und Hinweise der Lehrkräfte führten zu weiteren sprachlichen Vereinfachungen, ohne, dass der Sinngehalt gegenüber den früheren Versionen entstellt wurde.

Für die Schülerinnen und Schüler der Jahrgangsstufen 1 und 2 kam ein strukturiertes Interview zum Einsatz, bei dem deutlich einfacher formulierte, aber dem erstellten Fragebogen entsprechende Fragen mündlich gestellt und von den Interviewern entweder auf Band aufgenommen oder schriftlich festgehalten wurden. Um den entwicklungspsychologischen Voraussetzungen der jüngeren Schülerinnen und Schüler gerecht zu werden (Ermüdung, Konzentrationsmangel, Unvertrautheit mit solchen Situationen, Probleme, längere Zeit still zu sitzen etc.), wurde die Befragung mit ihnen deutlicher kürzer gehalten. So dauerte die mündliche Befragung der Erst- und Zweitklässler ca. 20 bis 25 Minuten und wurde nicht im Gruppenverband, sondern als Einzelgespräch mit den jeweiligen Schülerinnen und Schülern in einem separaten Raum durchgeführt. Die Auswahl der Schülerinnen und Schüler zum Einzelgespräch erfolgte entweder per Zufall - die Schülerinnen und Schüler, die sich zuerst gemeldet hatten, wurden berücksichtigt- oder wurden von den Klassenlehrerinnen und -lehrern den Interviewern zugeführt. Die schriftliche Befragung der Dritt- und Viertklässler erfolgte dagegen im Gruppenverband.

Für alle Stichproben wurde sichergestellt, dass eine Elterneinwilligung vorlag. Der Rücklauf bzw. die erteilte Zustimmungsrate war beeindruckend hoch; nach Information der jeweiligen Lehrkräfte hatten fast alle Eltern ihre Zustimmung gegeben.

Den Schülern wurde vor der Befragung die standardisierte Instruktion gegeben, die Fragen möglichst ehrlich und eigenständig zu beantworten, bei Unverständnis die Hilfe der anwesenden Interviewer per Handzeichen einzufordern. Fragen, die auf sie in nicht zutrafen, sollten die Schülerinnen und Schüler dann übergehen. Der Fragebogen für die Schülerinnen und Schüler war in deutscher Sprache verfasst. Um individuelle Einschätzungen und Meinungen zu erhalten, wurde der Fragebogen den nebeneinander sitzenden Schülerinnen und Schülern in einer A- und B-Version ausgeteilt, die zwar inhaltlich identisch waren, sich jedoch in der Reihenfolge der Fragen deutlich unterschieden. Dadurch konnte weitestgehend gewährleistet werden, dass die Schülerinnen und Schüler nicht voneinander abschrieben, sondern eigene Meinungen bekundeten. Während der Befragung waren die Lehrkräfte weitestgehend an der Befragung unbeteiligt, um eventuellen Verzerrungen in der Meinungsbildung der Schülerinnen und Schüler gegenüber 
dem „Islamischen Religionsunterricht“ und auch Loyalitätsgefühle gegenüber den unterrichtenden Lehrkräften nicht entstehen zu lassen.

Stichprobenkennzeichnung:

An der Befragung der ersten Welle, die im Zeitraum von Oktober 2005 bis Ende Februar 2006 erfolgte, nahmen 214 Schülerinnen und Schüler aus 10 Grundschulen teil. Von diesen waren 105 Jungen und 108 Mädchen; zwei machten keine Angaben zum Geschlecht. Der weitaus größte Teil der Schülerinnen und Schüler $(91 \%)$ ist in Deutschland geboren; rund $5 \%$ sind in der Türkei geboren und weitere rund $4 \%$ sind in einem anderen Land (außer Deutschland oder der Türkei) geboren. Berichtet werden hier quantitative Ergebnisse der Erhebungen aus den dritten (110 Schüler) und vierten Klassen (70 Schüler).

\subsection{Muslimische Schüler und ihre Akkulturationsbelastungen:}

Der Übergang von Elternhaus zur Schule bzw. der Schuleintritt ist generell für Kinder in der ersten Phase ein kritisches Lebensereignis, das potenziell stressverursachend ist. Dabei stehen Schülerinnen und Schüler mit Migrationshintergrund vor der besonderen Herausforderung, das doppelte Verhältnis, einerseits zur eigenen Ethnie, andererseits zur Aufnahmegesellschaft, eigenaktiv gestalten zu müssen. Hierbei lassen sich kulturpsychologischen Forschungen folgend in idealisierter Form vier Optionen unterscheiden, wie dieses Verhältnis gestaltet werden kann: Integration, Assimilation, Separation und Marginalisierung. ${ }^{30}$

Während bei Integration und Assimilation Handlungsoptionen stärker auf die aufnehmende Gesellschaft bezogen sind, wobei Integration zugleich Bezüge zur Herkunftskultur bzw. zur eigenen Ethnie stärker berücksichtigt, ist Separation durch eine stärkere Abgrenzung zur aufnehmenden Gesellschaft bei gleichzeitiger Hinwendung zur eigenen Ethnie und schließlich Marginalisierung durch eine Abgrenzung sowohl von dem eigenen ethnischen Hintergrund als auch durch eine Ablehnung von Beziehungen zur Mehrheitsgesellschaft gekennzeichnet. Dabei können diese Optionen bereichsspezifisch variieren (bspw. können Migranten sprachlich gut integriert sein und dennoch wenige Sozialkontakte zu Mehrheitsgesellschaft haben). Bezugnehmend auf dieses Modell wurde versucht, anhand einiger ausgewählter Bereiche die Akkulturationshaltungen von Schülern zu erfassen.

Hier werden exemplarisch die einzelnen Bereiche bzw. Frageformat und Antwortverhalten aufgezeigt:

30 Vgl. für eine ausführliche Darstellung vgl. Bourhis et al. (1997). 
Tabelle 1: Akkulturationsorientierungen der dritten und vierten Klassen

\begin{tabular}{|c|c|c|c|c|}
\hline Orientierung & Itemwortlaut & $\begin{array}{l}\text { stimmt } \\
\text { sehr }\end{array}$ & manchmal & $\begin{array}{l}\text { stimmt } \\
\text { nicht }\end{array}$ \\
\hline & & \multicolumn{3}{|c|}{ (Angaben in Prozent) } \\
\hline \multirow{3}{*}{ Integration } & $\begin{array}{l}\text { Ich möchte gut deutsch sprechen können } \\
\text { und auch türkisch nicht vergessen. }\end{array}$ & 83.2 & 8.6 & 8.1 \\
\hline & $\begin{array}{l}\text { Mir ist beides wichtig, türkische und } \\
\text { deutsche Freunde zu haben. }\end{array}$ & 73.0 & 18.4 & 8.6 \\
\hline & $\begin{array}{l}\text { Meine Familie sollte sowohl die türkische } \\
\text { Lebensweise bewahren und auch die } \\
\text { deutsche annehmen. }\end{array}$ & 40.7 & 29.7 & 29.7 \\
\hline \multirow{4}{*}{ Assimilation } & & & & \\
\hline & $\begin{array}{l}\text { Ich möchte auch in meiner Familie } \\
\text { deutsch sprechen und nicht türkisch. }\end{array}$ & 22.8 & 28.3 & 48.9 \\
\hline & $\begin{array}{l}\text { Ich möchte eher deutsche Freunde ha- } \\
\text { ben als türkische. }\end{array}$ & 17.2 & 24.7 & 58.0 \\
\hline & $\begin{array}{l}\text { Meine Familie sollte leben wie Deutsche } \\
\text { und nicht wie Türken. }\end{array}$ & 9.8 & 6.3 & 83.9 \\
\hline \multirow{4}{*}{ Separation } & $\begin{array}{l}\text { Ich spreche lieber türkisch; deutsch } \\
\text { spreche ich nur, wenn es unbedingt nötig } \\
\text { ist. }\end{array}$ & 49.7 & 31.6 & 18.7 \\
\hline & $\begin{array}{l}\text { Für mich ist es wichtiger, türkische } \\
\text { Freunde zu haben als deutsche. }\end{array}$ & 31.1 & 31.7 & 37.2 \\
\hline & $\begin{array}{l}\text { Meine Familie sollte nur ihre türkische } \\
\text { Lebensart behalten. }\end{array}$ & 71.5 & 17.3 & 11.2 \\
\hline & $\begin{array}{l}\text { In meiner Familie soll alles bleiben, wie } \\
\text { es in der Türkei war. }\end{array}$ & 72.7 & 22.4 & 4.9 \\
\hline \multirow{3}{*}{ Marginalisierung } & $\begin{array}{l}\text { Ich möchte weder mit Türken noch mit } \\
\text { Deutschen befreundet sein. }\end{array}$ & 14.3 & 14.3 & 71.4 \\
\hline & $\begin{array}{l}\text { Meine Familie sollte ihre Lebensweise } \\
\text { aufgeben, aber auch die deutsche nicht } \\
\text { annehmen. }\end{array}$ & 17.3 & 11.7 & 70.9 \\
\hline & $\begin{array}{l}\text { Weder das türkische noch das deutsche } \\
\text { Familienleben ist gut. }\end{array}$ & 31.8 & 20.5 & 47.7 \\
\hline
\end{tabular}

Vorsichtig gedeutet, können die Ergebnisse einen Hinweis geben, wie „integrationsoffen" muslimische Familien und Schülerinnen und Schüler sind, wobei jedoch im Rahmen dieser Erhebung nicht ausdrücklich erfasst wurde, aufgrund welcher eigener Erfahrungen diese Einstellungen favorisiert werden. Die untere Abbildung verdeutlicht die einzelnen Dimensionen in ihren Mittelwerten, wobei die jeweiligen Itemwerte addiert und durch die Anzahl der Items dividiert wurden. 
Abbildung 1: Ausprägung der jeweiligen Akkulturationsorientierungen

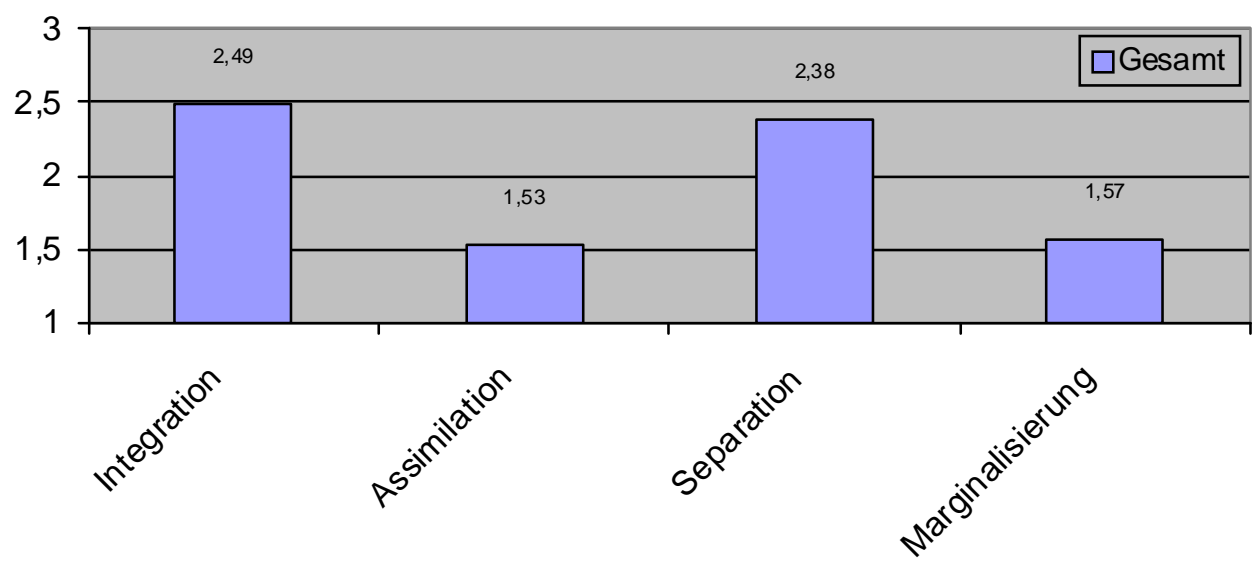

In den untersuchten Klassen bildet die Orientierung in Richtung Integration die stärkste Dimension, gefolgt von eher separationistischen Tendenzen. D.h. in ihrer Beziehungsgestaltung zu relevanten Aspekten der Mehrheitsgesellschaft möchten die Schülerinnen und Schüler in erster Linie sowohl Bezüge zu ihrer eigenen familialen Tradition, als auch Bezüge zu Deutschen haben. Was Schülerinnen und Schüler jedoch auf jeden Fall ablehnen, ist eine eindeutige Assimilationshaltung, d.h. die Aufgabe der eigenkulturellen Bezüge und eine völlige Identifikation mit mehrheitskulturellen Aspekten. Und auch die Haltung der Marginalisation, d.h. eine skeptische Haltung zur eigenen wie der Mehrheitskultur zugleich, wird deutlich abgelehnt. Zum besseren Verständnis der Daten ist anzuführen, dass diese Orientierungen keine Ausschlussverhältnisse anzeigen, d.h. die befragten Schülerinnen und Schüler sich nicht bspw. entweder für Integration oder Assimilation etc. zu äußern hatten, sondern für sich quantitativ diese Orientierungen gewichten sollten. Die ebenfalls recht hohe Ausprägung der Dimension „Separation“ ist vor dem entwicklungspsychologischen Hintergrund zu verstehen, dass in dieser Altersphase für Kinder die Zugehörigkeit zu einer „Wir-Gruppe“ und die Abgrenzung von den „Anderen“ für die eigene Identitätsentwicklung bedeutsam ist. Es ist kein unveränderliches Persönlichkeitsmerkmal, sondern abhängig von eigenen Erfahrungen mit und Kenntnissen über den „Anderen“. 
Ergebnisse zum wahrgenommenen Schulklima31

Die Schülerinnen und Schüler wurden um ihre Einschätzung bezüglich des „Islamischen Religionsunterrichts“ und ihrer Beziehung zu ihrer Religionslehrkraft gebeten. Ausdrücklich wurde Schülerinnen und Schülern versichert, es gäbe hierbei kein „richtig“ und „falsch“, sondern ausschließlich ihre Meinung sei relevant. Dabei sind u. a. folgende Dimension des Schul- bzw. Unterrichtsklimas gemessen worden: Fürsorglichkeit des Lehrers, Zufriedenheit der Schüler mit dem Unterricht sowie das Klima in der Kasse.

Tabelle 2: Fürsorglichkeit des Lehrers:

\begin{tabular}{|c|c|c|c|c|}
\hline \multirow[t]{2}{*}{ Dimension } & Itemwortlaut & oft & manchmal & selten \\
\hline & & \multicolumn{3}{|c|}{ Angaben in Prozente } \\
\hline \multirow{8}{*}{$\begin{array}{l}\text { Fürsorglichkeit } \\
\text { des Lehrers }\end{array}$} & $\begin{array}{l}\text { Wenn die Schüler Fragen an den Lehrer } \\
\text { haben, dann hat er auch Zeit für sie }\end{array}$ & 52.5 & 38.1 & 9.4 \\
\hline & Der Lehrer hilft uns wie ein Freund & 74.2 & 19.4 & 6.5 \\
\hline & $\begin{array}{l}\text { Unser Lehrer kümmert sich um die } \\
\text { Probleme der Schüler }\end{array}$ & 75.0 & 19.4 & 5.6 \\
\hline & $\begin{array}{l}\text { Der Lehrer achtet zuwenig darauf, wie } \\
\text { sich die Schüler fühlen }\end{array}$ & 29.1 & 28.6 & 42.3 \\
\hline & $\begin{array}{l}\text { Der Lehrer hilft jedem Schüler, der } \\
\text { Schwierigkeiten mit seiner Arbeit hat }\end{array}$ & 75.6 & 16.7 & 7.8 \\
\hline & $\begin{array}{l}\text { Im Islamunterricht reden wir über Fra- } \\
\text { gen, die für uns wichtig sind }\end{array}$ & 69.9 & 20.8 & 9.3 \\
\hline & $\begin{array}{l}\text { Der Lehrer bemüht sich, die Wünsche } \\
\text { der Schüler zu erfüllen }\end{array}$ & 48.9 & 39.0 & 12.1 \\
\hline & $\begin{array}{l}\text { Unser Lehrer ist bereit, mit uns zu } \\
\text { reden, wenn uns etwas nicht gefällt }\end{array}$ & 60.7 & 28.1 & 11.2 \\
\hline
\end{tabular}

Die Ergebnisse zeigen, dass die Schülerinnen und Schüler ihre Religionslehrkräfte im allgemeinen als recht fürsorglich und verständnisvoll erleben; allenfalls rund 10\% der Schülerinnen und Schüler zeigen sich mit den Lehrkräften unzufrieden. Des weiteren zeigen die Ergebnisse, dass rund zwei Drittel der Schülerinnen und Schüler mit dem durchgeführten Unterricht ziemlich zufrieden sind; allenfalls eine eher kleine Minderheit von 10 bis 15\% drückt ihr Unbehagen zum Verlauf des Unterrichts aus.

Auch die Zufriedenheit der Schülerinnen und Schüler mit dem „Islamischen Religionsunterricht“ fällt sehr deutlich aus; hier zeigte sich rund zwei Drittel der

31 Das Schul- bzw. Unterrichtsklima wurde in einer etwas modifizierten und sukzessive adaptierten Version des LASSO (Landauer Skalen zum Sozialklima) erhoben. Damit wird versucht, zu erfassen, wie Schüler ihre Lernumwelt wahrnehmen, die ein bedeutender Prädiktor für den Lernerfolg des Schülers bildet. Vgl. Saldern, M.V. \& Littig, K.E. (1987). Landauer Skalen zum Sozialklima. Beltz: Weinheim. 
Befragten sowohl mit den Inhalten als auch mit dem Ablauf des Unterrichts recht einverstanden.

Da der Zweck des Religionsunterrichts vorrangig darin besteht, sowohl das Wissen über religiöse Inhalte zu mehren bzw. vorhandenes Wissen zu vertiefen, wurde ausdrücklich danach gefragt, wie viel die Schülerinnen und Schüler aus ihrer Sicht im Unterricht zu einzelnen Aspekten der Religion dazugelernt haben.

Tabelle 4: Ergebnisse gezielter Fragen zum Wissenszuwachs durch den Islamunterricht

\begin{tabular}{|l|l|l|l|l|}
\hline \multirow{2}{*}{ Itemwortlaut } & \multicolumn{3}{l|}{ Angaben in Prozent } & Gar nicht \\
\cline { 2 - 5 } & $\begin{array}{l}\text { Ja, sehr } \\
\text { viel }\end{array}$ & Ja, etwas & $\begin{array}{l}\text { Nur ein } \\
\text { wenig }\end{array}$ & 1.5 \\
\hline $\begin{array}{l}\text { Im Islamunterricht habe ich etwas über } \\
\text { den Propheten Mohammed gelernt. }\end{array}$ & 74.8 & 17.5 & 6.3 & 5.2 \\
\hline $\begin{array}{l}\text { Im Islamunterricht habe ich einiges über } \\
\text { den Koran gelernt. }\end{array}$ & 53.3 & 36.2 & 5.2 & 5.2 \\
\hline $\begin{array}{l}\text { Im Islamunterricht habe ich einiges über } \\
\text { die islamische Geschichte gelernt. }\end{array}$ & 61.6 & 26.5 & 6.6 & 5.2 \\
\hline
\end{tabular}

Aus der subjektiven Sicht der Schülerinnen und Schüler hat der Religionsunterricht zu einem beträchtlichen Wissenszuwachs geführt: Fast $90 \%$ (,sehr viel“" und „etwas“ zusammen betrachtet) bekundeten, durch den Unterricht Wissen zu relevanten Aspekten des Islams (wie etwa Kenntnisse über den Propheten Mohammed, über die islamische Geschichte, über den Koran etc.) vermittelt bekommen zu haben. Lediglich für etwa $10 \%$ der befragten Schülerinnen und Schüler hat der bisherige „Islamische Religionsunterricht“ dagegen kaum oder gar nicht zu einem Wissenszuwachs geführt. Aus der Sicht der Schülerinnen und Schüler scheinen die stärksten Wissensimpulse im Hinblick auf die Kenntnisse über den Propheten Mohammed und dann im Hinblick auf die islamische Geschichte resultiert zu sein.

Bei der Frage, ob andere Religionen genauso wichtig seien wie der Islam, gaben rund $61 \%$ der Schülerinnen und Schüler eine eindeutig befürwortende Haltung, knapp 18\% hatte eher eine einschränkende Haltung und rund 20 lehnten dies jedoch ab bzw. drückten aus, dass andere Religionen für sie nicht genauso wichtig wie der Islam seien. Die weitergehende Frage, ob sie sich mehr Kenntnisse auch über andere Religionen wünschten, befürworteten rund ein Drittel eindeutig, ein Drittel gab an, sich manchmal mehr Kenntnisse über andere Religionen zu wünschen. Knapp $12 \%$ lehnten aber mehr Kenntnisse eher ab; rund $21 \%$ wünschte explizit keine Kenntnisse über andere Religionen.

Diese Ergebnisse zeigen eindeutig, dass für die Schülerinnen und Schüler des Islamunterrichts die Beschäftigung mit dem Islam keineswegs eine selbstgenügsame Haltung bedeutet, sondern sie weitestgehend offen für Kenntnisse anderer Religionen sind. 
Die bisher berichteten Schülerergebnisse zusammenfassend lässt sich also festhalten, dass Schülerinnen und Schüler des islamischen Religionsunterrichts in keiner Weise antiintegrative Tendenzen aufweisen, sondern dass sie primär die Akkulturationsstrategie der Integration favorisieren. Diese Aussage wird auch durch die Daten der Eltern, die hier aus Platzgründen nicht berichtet wurden, bestätigt.

Ferner zeigt sich, dass die bisherige Form des Islamunterrichts eine überwiegend positive Resonanz und Akzeptanz findet, gleichwohl Schülerinnen und Schüler wie auch Eltern einige Verbesserungspotenziale ausfindig machen.

Zwar lassen sich aus den vorliegenden Daten im engeren wissenschaftlichen Sinne keine Ursache - Wirkungs- Richtungen festmachen, die die bedeutsame Frage klären könnten, ob allein der Besuch des Islamunterrichts zu einer besseren Integration führt. Zumindest kann jedoch festgehalten, dass sowohl die befragten muslimischen Schüler wie auch deren Eltern (deren Daten hier aus Platzgründen nicht berichtet wurden) mit dem Angebot und der Durchführung des Islamischen Religionsunterrichts recht zufrieden sind, was ihrer prinzipiell hohen integrationsorientierten Haltung weiter förderlich sein könnte.

\section{Literatur}

Akbulut, D.: Türkische Moslems in Deutschland. Ein religionssoziologischer Beitrag zur Integrationsdebatte. Ulm 2003

Baumann, U.: Eine Welt — Viele Religionen. Christen und Muslime in Deutschland. In: Baumann,U. (Hrsg.): Islamischer Religionsunterricht. Grundlagen, Begründungen, Berichte, Projekte, Dokumentationen (S. 11-33). Frankfurt 2001

Bourhis, R. Y. Moise, C. L: Perreault, S. \& Senécal, S. : Immigration und Multikulturalismus in Kanada: Die Entwicklung eines interaktiven Akkulturationsmodells. In: Mummendey, A./Simon, B.: Identität und Verschiedenheit. Zur Sozialpsychologie der Identität in komplexen Gesellschaften (S. 63-108). Bern 1997

Bucher, A.: Religiosität verstehen. In: Frey, D./Hoyos, C. G. (Hrsg.): Psychologie in Gesellschaft, Kultur und Umwelt (S. 253-258). Weinheim 2005

Bucher, A.: Psychologische Aspekte neureligiöser Bewegungen. In: Frey, D./ Hoyos, C. G. (Hrsg.): Psychologie in Gesellschaft, Kultur und Umwelt (S. 259-265). Weinheim 2005

Büttner, F.: Islamischer Fundamentalismus- eine Herausforderung für den Westen? In: Busch, R. (Hrsg.): Integration und Religion. Islamischer Religionsunterricht an Berliner Schulen (S. 90-111). Berlin 2000

Heitmeyer, W./Müller, J./Schröder, H.: Verlockender Fundamentalismus. Türkische Jugendliche in Deutschland. Frankfurt 1997

Luhmann, N.: Funktion der Religion. Frankfurt 1982 
Müller, P.: (Religions-)Pädagogische Überlegungen. In: Baumann, U. (Hrsg.): Islamischer Religionsunterricht. Grundlagen, Begründungen, Berichte, Projekte, Dokumentationen (S. 163-181). Frankfurt 2001

Sackmann, R.: Türkische Muslime in Deutschland - Zur Bedeutung der Religion. Zeitschrift für Türkeistudien, Heft 1+2, 187-205. 2001

Saldern, M.V./Littig, K.E.: Landauer Skalen zum Sozialklima. Beltz 1987

Schiffauer, W.: Die Migranten aus Subay. Türken in Deutschland. Eine Ethnographie. 1991

Stöbe, A.: Die Bedeutung des Islam im Sozialisationsprozess von Kindern türkischer Herkunft und für Konzepte interkultureller Erziehung. Diss. Gesamthochschule Essen. 1998

Tan, D.: Zur Rolle der Religion in der Erziehung. In Analysen. Arbeitskreis Neue Erziehung (Hg.): Erziehung - Sprache - Migration. Gutachten zur Situation türkischer Familien (S. 37-92). Berlin 1999

Zirker, H.: Islam: theologische und gesellschaftliche Herausforderungen. Düsseldorf 1992 

Den Islam im Unterricht lehren 


\section{Ali Özgur Özdil: Lehrtradition, Vorbild im Glauben versus neue muslimische Fachdidaktik und Moscheepädagogik}

In meinem Beitrag geht es um die Rolle der muslimischen Religionslehrerinnen und -lehrer als Vorbilder für ihre muslimischen Schülerinnen und Schüler. Die Erwartungen an sie sind sehr hoch, sodass sie sich der kritischen Frage stellen müssen, ob sie diesen Erwartungen gerecht werden können oder müssen.

Der deutsche Staat, in dessen Dienst sie durch den Islamunterricht getreten sind oder - sofern sie sich noch in der Ausbildung befinden - treten werden, erwartet von ihnen die Erteilung einer den Interessen des Staates entsprechenden Unterrichts. Die islamischen Verbände wiederum, die seit Jahrzehnten islamischen Religionsunterricht einfordern und über diesen verhandeln, haben ebenfalls Erwartungen, ebenso die muslimischen Eltern und Schülerinnen und Schüler, worauf ich weiter unten eingehen möchte.

Es sei vorweggeschickt, dass noch in keinem Bundesland Islamischer Religionsunterricht gemäß Art. 7 (3) GG erteilt wird. Demnach ist der gegenwärtige Islamunterricht ein islamkundlicher Unterricht, der nicht gemäß den Grundsätzen der islamischen Religionsgemeinschaft erteilt wird. Er entspricht also nicht den tatsächlichen Erwartungen der muslimischen Antragsteller. So herrscht auch eine 
Unsicherheit bezüglich der Lehrerinnen und Lehrer, die diesen Unterricht erteilen, da ein religionskundlicher Unterricht - rechtlich gesehen - das Bekenntnis und die Mitgliedschaft zu einer Religionsgemeinschaft nicht voraussetzt. Das wiederum führt zu einem Spannungsfeld zwischen Lehrerschaft und Religionsgemeinschaft. Sollte es sich jedoch um einen Religionsunterricht im Sinne des Art. 7 (3) GG handeln, würde vorausgesetzt werden, dass die Lehrerinnen und Lehrer Muslime sind. Der Islamunterricht - im Gegensatz zum Islamischen Religionsunterricht umgeht bisher dieses rechtliche Problem. Dennoch ist den kritischen Aussagen von Muslimen zu entnehmen, dass der gegenwärtige Unterricht eine „Mogelpackung" sei und unter der Lehrerschaft auch Atheisten oder Aleviten vorhanden seien. Dieser Umstand wird damit verglichen, dass ein katholischer Religionsunterricht z.B. von einem Atheisten oder einem evangelischen Christen erteilt werden dürfte.

Ich verweise auf diese Problematik deswegen, weil der Religionsunterricht gemäß Grundgesetz zwar ein „ordentliches Lehrfach“ ist, aber dennoch Eltern das Recht gibt, ihre Kinder bis zum 14. Lebensalter davon abzumelden. Ab dem 14. Lebensalter entscheiden die Schülerinnen und Schüler selbst, ob sie am Religionsunterricht oder an einem Alternativfach wie etwa Ethik oder Philosophie teilnehmen möchten. Da der gegenwärtige Islamunterricht in der Regel die Klassen 1-6 betrifft und sein Erfolg auch davon abhängt, ob er eine hohe Akzeptanz unter der muslimischen Elternschaft genießt, kommen die Betreiber dieses Unterrichts nicht darum herum, vor allem die Eltern dafür zu gewinnen. Schließlich ist eine Mindestzahl von muslimischen Schülerinnen und Schülern notwendig, damit Unterricht überhaupt stattfinden kann.

Ich persönlich bin der Ansicht, dass jeder Unterricht mit der Person des Lehrers steht oder fällt. Fakt ist, dass die Schülerinnen und Schüler häufig jenen Unterricht am meisten mögen, wo sie auch den Lehrer mögen.

\section{Lehrtradition}

Ich habe mehrere Lehrerinnen und Lehrer in verschiedenen Bundesländern nach den Erwartungen der Eltern und Schülerinnen und Schüler befragt. Es hat sich herausgestellt, dass die meisten Eltern zwar einen Religionsunterricht für ihre Kinder wünschen, aber weder wissen, was genau ein schulischer Religionsunterricht ist noch den Unterschied zwischen Islamunterricht und islamischem Religionsunterricht kennen. Dort, wo es keinen Unterricht für muslimische Schülerinnen und Schüler gibt, nehmen die Kinder entweder am christlichen Religionsunterricht oder an gar keinem Religionsunterricht teil. Nicht selten wird der Religionsunterricht im Stundenplan auch parallel zum muttersprachlichen Türkischunterricht gelegt. Die Eltern sind - das betrifft nicht nur die muslimischen Eltern kaum darüber informiert, dass sie ihre Kinder vom Religionsunterricht abmelden können. 
Als Vater von zwei schulpflichtigen Kindern, die beide am Hamburger „Religionsunterricht für alle" teilnehmen, weiß ich aus eigener Erfahrung, dass die Eltern während des Elternabends über den Religionsunterricht informiert werden, jedoch nicht über das Recht auf Abmeldung.

Dort, wo islamischen Religionsinhalte unterrichtet werden und Eltern ihre Kinder anmelden, haben Eltern und gelegentlich auch Schülerinnen und Schüler einen klassischen Unterricht im Sinn. Sie denken, dort werde das Lesen des Korans gelehrt und das rituelle Gebet vermittelt. Diese Erwartungen sind erst einmal natürlich, da ein Islam ohne Koran und Gebet undenkbar wäre. Dabei geht es im Schulunterricht weniger um das „Wie“, sondern vielmehr um das „Warum“, also um die Sinnfrage. Für die Praxis sind eigentlich die Eltern verantwortlich, und wenn sie sich dazu nicht in der Lage sehen, gibt es schließlich die Moscheen als Orte gelebter Religion. Sollte man den Umfragen jedoch glauben, so gehen gerade einmal 22\% der muslimischen Kinder in Deutschland zu einem Korankurs. ${ }^{32} \mathrm{Da}$ mit wäre der schulische Unterricht auch für die meisten muslimischen Kinder der erste Ort, wo sie mit ihrer eigenen Religion in Kontakt treten. Für jene, die auch in den Familien und in den Moscheen nach den Islam leben, wäre er eine sinnvolle Ergänzung.

Den muslimischen Eltern muss klar werden, dass - aufgrund der unterschiedlichen Lernort und Lernsituationen - die Lehre in der Moschee nicht genügen kann. Darauf werde ich in Punkt 4 näher eingehen. Was in den Moscheen passiert, ist eben weniger Religionspädagogik oder Unterricht, sondern vielmehr Belehrung. Die klassische Lehrtradition, die für die religiöse Praxis wichtig ist, darf dennoch nicht außer Acht gelassen werden. So muss auch muslimischen Lehrerinnen und Lehrern ein Mindestmaß an Arabischkenntnissen abverlangt werden sowie der korrekte Umgang mit den islamischen Quellen. Lehrerinnen und Lehrer, die in der Lage sind den Koran zu lesen und die einige Suren auswendig können, sind für die Schülerinnen und Schüler eher eine Autorität in Fragen des Islam als jene, die weder das eine noch das andere können.

\section{Vorbild im Glauben}

Lehrerinnen und Lehrer sind Vorbilder. Meine bereits erwähnte Umfrage hat ergeben, dass es für ihre Schülerinnen und Schüler wichtig ist zu wissen, ob ihre Lehrerinnen und Lehrer an Allah glauben oder ob sie beten oder fasten. Es gibt Schülerinnen und Schüler, die ihre Lehrerinnen und Lehrer direkt danach fragen. Es gibt also bei einigen Schülerinnen und Schülern gewisse Erwartungen, an denen sie ihre Lehrerinnen und Lehrer messen. Demnach ist ein Lehrer bzw. eine Lehrerin für die Schülerinnen und Schüler niemals neutral. Sie sind Mann oder

32 Vgl. Nachrichten 2008, unter: http://www.islamarchiv.de/akver/in_online.html (abgerufen am 30.03.09) 
Frau, Muslim/in oder nicht und Türke, Araber usw. Niemand kann, was sein Geschlecht, seinen Glauben, seine Nationalität oder etwa seinen Beruf betrifft, neutral sein. Daher habe ich am Anfang meines Beitrages die Frage gestellt, ob die Lehrerinnen und Lehrer für Islamunterricht den Erwartungen (des Staates, der islamischen Verbände, der Eltern und der Schülerinnen und Schüler) gerecht werden können bzw. müssen. Meines Erachtens müssen sie gewissen Erwartungen gerecht werden, denn für den Fall, dass sie als islamische Autorität versagen, müssen wir mit Abmeldungen vom Islamunterricht rechnen. Dies wiederum würde das Projekt Islamunterricht gefährden. Das Projekt an sich ist - so lautet das politische Ziel z.B. in Niedersachsen - der Weg zu einem Islamischen Religionsunterricht gemäß Art. 7 (3) GG. Damit das Projekt gelingt, hilft es uns nicht, nur die positiven Seiten zu erwähnen, die es durchaus auch gibt. So existiert das Problem, dass Lehrerinnen, die ohne Kopftuch unterrichten, mich während einer Fortbildung gefragt haben, ob die muslimischen Eltern sie, denn als Muslimin akzeptieren würden. Ich hatte auch muslimische Lehrerinnen und Lehrer in der Fortbildung, die das islamische Glaubenszeugnis (die Schahada) nicht kannten und die zugaben, Alkohol zu trinken und Schweinefleisch zu essen. Viele gaben an, nicht zu beten. Einige meinten sogar, dass sie den Kindern nicht erzählen würden, dass man im Islam beten müsse oder dass Alkohol verboten sei.

Auch wenn von Seiten der Schule als Einrichtung des religionsneutralen Staates die Religiosität der Lehrerinnen und Lehrer als ihre Privatsache betrachtet wird, für die Eltern und die Schülerinnen und Schüler ist wichtig, ob die Lehrerinnen und Lehrer tatsächlich muslimisch sind oder nicht. Das zeigt sich eben in der Praxis. Schließlich müssen Lehrerinnen und Lehrer auch glaubwürdig sein. Vorbildlichkeit, Autorität und Glaubwürdigkeit - dies gilt auch für Eltern und Imame - sind demnach die Kernelemente für das Gelingen der religiösen Bildung. So erfuhr ich aus zwei Bundesländern, wie muslimische Lehrerinnen und Lehrer sich als „Kulturmittler“ bezeichneten, die den Schülerinnen und Schülern auch in Lebensfragen und bei familieninternen Problemen beratend halfen. Erst wenn sie das Vertrauen der Kinder genießen, ist dies möglich.

\section{Muslimische Fachdidaktik}

Damit meine Ausführungen nicht missverstanden werden: Ich entwickle Unterrichtsmaterialien für den Religionsunterricht, bilde Lehrerinnen und Lehrer fort und berate sie. Ich unterstütze die Schura Niedersachsen und die Schura Schleswig-Holstein bei ihren Bemühungen, Islamischen Religionsunterricht einzuführen, und möchte helfen, eine islamische Fachdidaktik zu entwickeln, die den Bedürfnissen der muslimischen Schülerinnen und Schülern entspricht.

Bei der muslimischen Fachdidaktik muss jedoch die Frage gestellt werden, ob sie auf den Islamunterricht oder den Islamischen Religionsunterricht bezogen ist. Auch wenn - wie gesagt - viele Eltern den Unterschied zwischen Islamunterricht 
und Islamischen Religionsunterricht nicht kennen und auch viele muslimische Lehrerinnen und Lehrer, die derzeit unterrichten, keinen Unterschied machen, sehe ich dennoch einen Unterschied zwischen einem islamkundlich vermittelten Unterricht und einem Fach, das als „konfessionell“ oder als „Bekenntnisfach“ bezeichnet wird, wobei die Bezeichnung „konfessionell“ in Bezug auf den Islam nicht passend ist. Ein allein vom Staat getragener Unterricht wie der Islamunterricht ist sicherlich nicht identisch mit jenem Unterricht, der ein in gemeinsamer Verantwortung und gleichberechtigt erteilter Religionsunterricht ist. Eines möchte ich hier in diesem Zusammenhang unbedingt unterstreichen: Auch ein islamkundlicher Unterricht kann gut sein, wenn die Lehrperson gut ist, wohingegen ein Religionsunterricht schlecht sein kann, wenn die Lehrperson die Anforderungen nicht erfüllt.

Rahmenpläne, Unterrichtsmaterialien (und damit sind nicht allein Religionsbücher gemeint), regelmäßige Fortbildung und vielleicht auch Beratungsmöglichkeiten sind wichtige Bedingungen für einen guten Unterricht. Am allerwichtigsten scheint mir allerdings die Ausbildung zu sein, in der eben die nötige Fachdidaktik gelernt wird. Die meisten Lehrerinnen und Lehrer, die derzeit Islam unterrichten, sind jedoch keine ausgebildeten Lehrerinnen und Lehrer für Islamunterricht. Ein gutes Konzept, mit der man bundesweit Werbung machen kann, reicht m. E. also alleine nicht aus.

Wenn man die Lehrerinnen und Lehrer für Islamunterricht fragen würde, ob sie eine Ausbildung in islamischer Fachdidaktik haben, würde das kein gutes Licht auf das Projekt werfen. Ebenso könnte man sie fragen, was für sie eine ,islamische Religionspädagogik“ ist. Da es sich für die Bildungslandschaft in Deutschland um ein neues Fach handelt, in der eine islamische Religionspädagogik erst entwickelt wird, sollte auch die kritische Frage gestellt werden, warum damit bis heute gewartet wurde, obwohl Islamischer Religionsunterricht seit Anfang der 1980er Jahre gefordert wird. Eine ganze Generation ist verstrichen, und wir stehen vor dem Dilemma, dass viele Lehrerinnen und Lehrer, die ich frage, diese Fragen nicht beantworten können. Was also ist muslimische Fachdidaktik? Mit anderen Worten: Wie vermitteln die Lehrerinnen und Lehrer die Unterrichtsinhalte? Diese Frage ist insofern mit Schwierigkeiten verbunden, weil für die Vermittlung des Unterrichts Materialien benötigt werden. Auch da sieht es bundesweit nicht so gut aus. Ich habe - wie mir später von einigen Lehrerinnen und Lehrer berichtet wurde - durch Fortbildungsseminare in Berlin, ${ }^{33}$ Baden-Württemberg (Mannheim) ${ }^{34}$ und in Österreich (Wien, Graz, Linz, Innsbruck, Salzburg) ${ }^{35}$ die Entwicklung von

\footnotetext{
33 Siehe http://www.iwb-

hamburg.de $/$ index.php?option $=$ com_content $\& v i e w=$ article\&id $=47 \&$ Itemid $=54$

34 Siehe http://www.iwb-

hamburg.de $/$ index.php?option $=$ com_content\&view $=$ article\&id $=63 \& I t e m i d=70$

35 Siehe http://www.iwb-

hamburg.de $/$ index.php?option $=$ com_content\&view $=$ article\&id $=64 \& I t e m i d=71$
} 
Materialien vorangetrieben, indem ich die von mir entwickelten Materialien vorgestellt und den Lehrerinnen und Lehrern gezeigt habe, wie diese entstehen und wie sie im Unterricht umgesetzt werden können. Meine Erfahrung war, dass bisher alle Lehrerinnen und Lehrer die Materialien für die Unterrichtsthemen selbst zusammenstellten oder einfach nur ein Religionsbuch benutzten. Gelegentlich wurde auch von Materialien für den christlichen Religionsunterricht profitiert. Es stellen sich uns also noch sehr viele Aufgaben.

Als Fazit kann ich jedoch ziehen, ohne dies verallgemeinern zu wollen, da es auch Ausnahmen gibt, dass eine für die Lehrerinnen und Lehrer für Islamunterricht klar definierte (muslimische) Fachdidaktik nicht vorhanden ist, sondern individuell ,islamisch“ gearbeitet wird. D.h. eine muslimische Lehrerin unterrichtet muslimische Kinder über ein islamisches Thema, und wir nennen das Ganze Islamunterricht, also einen Unterricht, der dem Islam entspricht. Das aber tun auch Eltern zu Hause oder Imame in der Moschee. Inwiefern hat das Ganze dann einen pädagogisch messbaren didaktischen und somit wissenschaftlichen Wert? Daher halte ich an dem „Neuen“ fest, dass nach einer neuen muslimischen Fachdidaktik gesucht wird, auf die sich Staat und Muslime einigen müssen, und zwar so, dass es den Bedürfnissen der muslimischen Schülerinnen und Schüler entspricht - vor allem was ihre Identitätsentwicklung in einer pluralistischen, säkularen Gesellschaft, in der Muslime aus aller Welt als Minderheit leben, betrifft.

Damit treffen wir nämlich den zentralen Punkt der Frage nach einer neuen Fachdidaktik. Wo soll wie, was vermittelt werden? Nicht in der Türkei oder in Ägypten, sondern in Deutschland, nicht in einer islamischen, sondern in einer überwiegend von Christen und Atheisten dominierten Gesellschaft, nicht in einem laizistischen, sondern in einem säkularen Staat soll das Ganze realisiert werden. Es ließen sich noch viel mehr historisch, kulturell sowie auf das politische und wirtschaftliche System bezogene gesellschaftlich relevante Differenzen nennen, die Berücksichtigt werden müssten, um das Neue, auf das Muslime hierzulande stoBen, entwickeln zu können. Denn sowohl wir (Muslime) müssen mit den genannten, von Land zu Land unterschiedlichen Bedingungen leben als auch die Mehrheitsgesellschaft, die sich nicht selten durch die Präsenz des Islam in dieser Gesellschaft in ihren Normen erschüttert sieht. Natürlich hat der Islam das Gesellschaftsbild verändert und mitgeprägt. Muslime wollen und dürfen aber auch hier leben, wie es der Islam von ihnen als Gläubige erfordert. Das Grundgesetz garantiert Religionsfreiheit und somit auch den Bau von Moscheen oder eben einen Islamischen Religionsunterricht. Diese Veränderungen im Stadtbild oder in der Schullandschaft können einerseits nicht reibungslos verlaufen und müssen andererseits als Realität akzeptiert werden.

Die neue Fachdidaktik muss also sowohl auf die Bedürfnisse der muslimischen Schülerinnen und Schüler als auch auf die Sensibilitäten in der Gesellschaft als Ganzes Rücksicht nehmen. Daher gestaltet sich vieles schwierig. Es wäre zu einfach zu sagen: Wir geben den muslimischen Kindern das, was sie zur Entwicklung 
einer gesunden islamischen Identität benötigen. Wir können genau definieren, was die sechs Glaubenselemente und die fünf Säulen des Islam sind. Wer an diese Elemente glaubt und diese Säulen erfüllt, ohne einen Zweifel an ihnen zu haben, ist ein gläubiger Muslim. Das ist der Konsens. Wie aber soll ich meinen Glauben in dieser Gesellschaft praktizieren, ist die Frage, der die Fachdidaktik nachgehen muss. Bin ich ein guter Muslim, obwohl ich nicht zum Freitagsgebet kann, weil mich mein Arbeitgeber nicht freistellt? Kann ich als Schüler oder Student an der Tankstelle arbeiten, wo ich auch Alkohol verkaufen muss?

Die Fachdidaktik muss sich also an Inhalten orientieren, die im Rahmenplan für den Islamunterricht vorkommen, diese aber nicht als theoretische Vorgaben behandeln, die von der Gegenwart und Gesellschaft isoliert sind. Daher benötigen wir auch Lehrerinnen und Lehrer, die mitten im Leben stehen und somit die Fragen ihrer Schülerinnen und Schüler kennen.

\section{Moscheepädagogik}

In Deutschland gibt es derzeit ca. 2500 Moscheen. In fast jeder Moschee werden Korankurse angeboten. So kommen die Moscheen den Wünschen muslimischer Eltern nach, den Kindern das Lesen des Korans und einige Suren für das rituelle Gebet beizubringen. Der Begriff der „Pädagogik“ in Zusammenhang mit einer Moschee ist insofern nicht fremd, als Moscheen früher - ähnlich wie die Kirchen - die zentralen Bildungseinrichtungen islamischer Gesellschaften waren (von der Schule bis zur Universität). Im Laufe des Säkularisierungsprozesses hat sich vielerorts die Schule von der Moschee gelöst und ist in den staatlichen Verantwortungsbereich übergegangen. Der schulische Religionsunterricht hat jedoch die Daseinsberechtigung der Moschee oder Kirche nicht überflüssig gemacht, denn es gibt weiterhin zusätzlich Kommunions- oder Konfirmandenunterricht oder eben Korankurse.

Obwohl die historische Realität zeigt, dass auch in Moscheen Bildung eine wichtige Rolle spielt, ist unter den gegenwärtigen Bedingungen zu fragen, ob das, was in Moscheen stattfindet, als „Pädagogik“ bezeichnet werden kann. Wenn wir Pädagogik in ihrer ursprünglichen griechischen Bedeutung als „Führung eines Kindes" verstehen, trifft dies sicherlich auf die Kurse in Moscheen zu. Messen wir die Kurse jedoch an der wissenschaftlichen Disziplin an Universitäten oder Schulen, dann ist dies in den meisten Moscheen mit einem großen Fragezeichen zu versehen.

Ich habe den Begriff der Moscheepädagogik als Äquivalent zur Kirchenpädagogik benutzt und auf eine bestimmte Moschee in Hamburg bezogen, in der ich selbst zwei Jahre lang mit muslimischen Kindern gearbeitet habe, aber auch als ein Angebot für Schulklassen, die in die Moschee kommen, um dort etwas über den Islam zu lernen; die Moschee also als Ort für pädagogisches Lernen. Als eine Studentin der Erziehungswissenschaft an der Universität Hamburg zu mir ins Institut 
kam, weil sie die Idee hatte, eine Examensarbeit über Moscheepädagogik zu schreiben, war sie lediglich auf eine einzige wissenschaftlich verwertbare Quelle gestoßen, in der der Begriff Pädagogik in Zusammenhang mit Moschee genannt wird, nämlich auf mein Buch „Wenn sich die Moscheen öffnen“ (vgl. Özdil 2002). Also auch auf diesem Gebiet muss sich einiges an Forschungsarbeit tun. Damit meine ich nicht die vielen Quellen aus den 1970er und 1980er Jahren, in denen es fast ausschließlich darum ging, Moscheevereine anzugreifen und Korankurse zu verdammen, statt sie ernsthaft in ihrer Bedeutung für die Erziehung muslimischer Kinder $\mathrm{zu}$ analysieren. Ich will auch nicht verschweigen, dass sich inzwischen etwas tut (vgl. Rauf 2008). Vielmehr meine ich, dass die Moschee als Lernort keine Konkurrenz zum schulischen Religionsunterricht ist, sondern eine sinnvolle Ergänzung sein kann, wenn sie gefördert wird, statt verteufelt zu werden.

\section{Schlusswort}

In Hamburg haben die Vertreter verschiedener Religionen eine „Didaktik der Religionen“ formuliert. ${ }^{36}$ Diese betrifft jedoch einen Religionsunterricht, in dem Schülerinnen und Schüler verschiedener Religionen, Konfessionen und Weltanschauungen sitzen. Dort heißt es: Wir leben in einer pluralistischen Gesellschaft mit Menschen unterschiedlicher Glaubensrichtungen. Auch an den Schulen gibt es Schülerinnen und Schüler, die von verschiedenen Religionen geprägt sind. Religionen wie Islam oder Buddhismus, die bisher als fremd empfunden wurden, sind nun die Religionen von den Mitschülerinnen und -schülern und deren Eltern, ja sogar von einigen Lehrerinnen und Lehrern. In einem Unterricht, der die

„unterschiedlichen religiösen Biographien seiner Schülerinnen und Schüler ernst nimmt, wird es vor allem darauf ankommen, miteinander nach Orientierungen im Füblen und Denken, im Glauben und Handeln zu suchen, die einen offenen Dialog über Grunderfahrungen und Grundbedingungen des Lebens ermöglichen und eine lebensfreundliche, menschenwürdige Zukunft für alle in einer endlichen Welt im Sinn haben" (aus dem Lehrplanentwurf Religion für die Grundschule in Hamburg).

Der Unterricht muss dazu beitragen, dass sich der Bezug bzw. das Verhältnis zu den Mitmenschen und ihren jeweiligen Religionen positiv verändern muss. Sie müssen nicht mehr als ferne, fremde Objekte betrachtet werden, sondern als unmittelbar nahe Subjekte des eigenen Lebens wahrgenommen werden.

Welche Folgerungen ergeben sich in der Thematisierung einer der Lehrkraft fremden Religion?

- Die andere Religion darf nicht allein ausgehend von dem alleinigen Wahrheitsanspruch der eigenen Religion vermittelt werden. Die eigene

36 Zur Situation des Religionsunterrichts in Hamburg habe ich mich bereits in verschiedenen Aufsätzen geäußert:, z.B. Özdil 2008, Özdil 2006 
Religion darf nicht als „Angebot“ an die Anhänger einer anderen Religion herangetragen werden. Dieses Denken schließt eine inhaltliche und didaktische Kürzung der anderen ein.

- Nur die authentische Antwort von Vertretern der Religionen kann helfen, sie in ihrem Selbstverständnis zu verstehen und zu beurteilen. Bei einer „Außensicht“ werden fremde Maßstäbe angelegt.

- Ein Gespräch mit kompetenten VertreterInnen der Religionen kann deutlich machen, wo die echten Schwerpunkte der Religionen liegen. Missverständnisse lassen sich so aufklären, Vorurteile werden abgebaut. (Beispiel: Kompetente Vertreter werden in die Klasse eingeladen; Gebetsstätten der Religionen werden besucht).

- Eine vorschnelle vergleichende Betrachtung von Religionen kann zu einer möglichen Nivellierung führen, wenn ein entsprechendes Fundament nicht vorhanden ist (Beispiel: Bibel $=$ Koran).

- Die Lehrkraft darf nicht die anderen Religionen aus der Sichtweise der eigenen Religion darstellen (Beispiele: Moschee $=$ Kirche der Muslime, Koran $=$ Bibel der Muslime).

- Sind in der Klasse Anhänger einer thematisierten Religion, sollte die unterrichtende Lehrkraft Zurückhaltung üben, wenn die Schülerinnen und Schüler über Praktiken ihrer Religion erzählen, die der Lehrkraft unbekannt sind.

Wenn wir die Situation muslimischer Schülerinnen und Schüler vor Augen haben, so sehen wir auch hier eine Vielfalt, die einerseits Probleme schaffen kann (etwa, wenn zu einem Sachverhalt unterschiedliche bzw. gegensätzliche Ansichten vertreten werden) oder eine Chance sein kann, um die Vielfalt und den Reichtum unter Muslimen zu thematisieren. Fakt ist, dass im Islamunterricht nicht nur Schülerinnen und Schüler sitzen, die alle türkischer Herkunft und hanafitisch geprägt sind. Darauf müssen Lehrerinnen und Lehrer Rücksicht nehmen, was ihnen die Arbeit jedoch nicht unbedingt erleichtern wird.

\section{Literatur}

Ceylan, Rauf Islamische Religionspädagogik in Moscheen und Schulen. Hamburg 2008

IWB Jahresbericht 2005, abrufbar unter: http://www.iwb-

ham-

burg.de/index.php?option $=$ com_content\&view $=$ article\&id $=47 \&$ Itemid $=54$

IWB Jahresbericht 2006, abrufbar unter: http:/ /www.iwb-

ham-

burg.de/index.php?option $=$ com_content\&view $=$ article\&id $=63 \&$ Itemid $=70$ 
IWB Jahresbericht 2007, abrufbar unter: http://www.iwbham-

burg.de/index.php?option $=$ com_content\&view $=$ article\&id $=64 \&$ Itemid $=71$

Nachrichten 2008, abrufbar unter:

http://www.islamarchiv.de/akver/in_online.html (abgerufen am 30.03.09)

Özdil, A. Ö.:Wenn sich die Moscheen öffnen. Münster 2002

Özdil, A. Ö.: Religiöse Identitätsbildung muslimischer Kinder im Rahmen des Hamburg Modells, in: Kaddor, Lamya: Islamische Erziehungs- und Bildungslehre. LIT Verlag Münster-Hamburg-Berlin-Wien-London-Zürich 2008

Özdil, A. Ö.: Religionsunterricht. In: Gerber, Uwe (Hrg.): Auf die Differenz kommt es an. Interreligiöser Dialog mit Muslimen. Leipzig 2006, S. 185-198 


\section{Katja Koch: Was sollen Lehrerinnen und Lehrer für den islamischen Religionsunterricht können?}

Der Beitrag stellt die relativ profane Frage: Was sollen Lebrerinnen und Lebrer können? und geht vor allem auf aktuelle Anforderungen an die Qualifikation von Lehrerinnen und Lehrern ein. Ich möchte die in der schulpädagogischen Forschung diskutierten Anforderungen des Lehrberufs verdeutlichen und betone daher die pädagogische Dimension religiöser Erziehung. Inhaltlich ist der Beitrag zunächst durch folgende drei allgemeine Thesen zum Können von Lehrerinnen und Lehrern strukturiert:

Diese lauten:

- Das Können der Lehrerinnen und Lehrer leitet sich aus den Funktionen von Schule ab.

- Das Können der Lehrerinnen und Lehrer wird von den Charakteristika des Lehrberufs determiniert.

- Das Können der Lehrerinnen und Lehrer muss auf „guten Unterricht“ zielen. Vor diesem Hintergrund versuche ich die Frage zu erläutern, welche Konsequenzen sich hieraus für das Können der Lehrerinnen und Lehrer für islamischen Religionsunterricht ableiten lassen. Eine Bemerkung vorweg: Ich bin keine Religionspädagogin, meine Überlegungen hierzu sind eher allgemeiner Art. Ich vertrete eine 
schulpädagogische Sicht der Dinge, die zudem noch empirisch gefärbt ist. Dies bedeutet, dass ich davon ausgehe, dass sich die Welt zwar nicht vollständig durch empirische Studien erklären lässt, wir sie jedoch ein Stück weit realistischer einschätzen können, wenn wir uns an deren Ergebnissen orientieren.

Ich komme nun zu meiner ersten These:

\section{Das Können der Lehrer leitet sich aus den Funktionen von Schule ab.}

Wenn wir eine Antwort auf die Frage nach dem für Lehrerinnen und Lehrer und notwendigen Können suchen, ist es zunächst einmal sinnvoll sich mit den Aufgaben von Schule zu befassen, da sich dieses Können innerhalb des dort abgesteckten Rahmens bewegt. Fend beschreibt in seiner Theorie der Schule, Schule als ein Zweck-Mittel-System, das darauf zielt, Lernprozesse für große Massen von Schülern zu organisieren. Schule schafft Lernvoraussetzungen, formuliert Lernziele, systematisiert Lernbedingungen und kontrolliert den Lernerfolg. Schule sichert so ein Wertesystem, das bestimmt wird von Unabhängigkeit, Leistung, Universalismus und Spezifität und das charakteristisch ist für moderne Industriegesellschaften (Fend 1980). Dabei erfüllt Schule drei Funktionen:

Sie hat zunächst die Aufgabe, die Schülerinnen und Schüler mit jenen Qualifikationen auszustatten, die sie später brauchen, um die Anforderungen im Arbeitsprozess erfüllen zu können. Die Qualifikationsfunktion der Schule beinhaltet dabei sowohl funktionale Qualifikationen, wie z.B. Lesen, Schreiben, Rechnen aber auch solche als extrafunktionale Qualifikationen bezeichnete Einstellungen und Fähigkeiten wie z.B. Fleiß, Ausdauer, Konzentrationsfähigkeit. Qualifikation meint also nicht nur den Erwerb von Fertigkeiten, sondern auch die Vorbereitung auf die Teilhabe am gesellschaftlichen Leben.

Die Selektions- und Allokationsfunktion der Schule verweist darauf, dass mit dem Besuch unterschiedlicher Schulformen die Vergabe unterschiedlicher Schulabschlüsse verknüpft ist und von diesen wiederum verschiedene Zuordnungen zu beruflichen Positionen abhängen. Anders als in ständisch gegliederten Gesellschaften, in denen die gesellschaftlichen Positionen nach solchen Kriterien wie Abstammung, Besitz oder Geschlecht vergeben werden, erfolgt in bürgerlichen, demokratisch verfassten Gesellschaften die Vergabe der unterschiedlichen mit Macht, Einkommen und Ansehen ausgestatteten gesellschaftlichen Positionen nach der individuellen Leistungsfähigkeit. Die Aufgabe Schüler nach ihrer erbrachten Leistung zu sortieren wird durch die Selektionsfunktion der Schule beschrieben. Die verschiedenen Berechtigungen zum Aufsteigen in die nächsthöhere Schulstufe, zum Übertritt in verschiedene Schultypen, zum Eintritt in bestimmte Positionen im Beschäftigungssystem, zur Aufnahme von Studien an Universitäten und Hochschulen wird auf Grund von gesellschaftlich normierten Leistungs- 
nachweisen in Form von Zeugnisse verliehen. Dies wird mit der Allokationsfunktion der Schule umschrieben.

Die Güte eines Schulsystems zeichnet sich dabei dadurch aus, wie gerecht diese Funktionen ausgeübt werden. Für das deutsche System lässt sich festhalten, dass es hier eine deutliche Koppelung von Allokation und sozialer Herkunft der Schüler gibt (vgl. Tillmann 2008, Bellenberg/Klemm 1995).

Belegen lassen sich dies u.a. am geringen Anteil ausländischer Kinder und Jugendlicher in prestigeträchtigen Schulformen. Ausländische Kinder und Jugendliche z.B. besuchen häufiger die Hauptschule und seltener das Gymnasium als deutsche Kinder und Jugendliche und sind im Vergleich zu ihren deutschen Altersgenossen insbesondere in der Sonderschule überrepräsentiert. Zwar profitiert auch diese Gruppe von der im Kontext der Bildungsexpansion gestiegenen faktischen Bildungsbeteiligung, der Abstand zu den gleichaltrigen deutschen Jugendlichen ist jedoch insbesondere im Bereich der höheren Abschlüsse noch beträchtlich. So ging z.B. im Zeitraum von 1984 bis 2003 der Anteil ausländischer Schülerinnen und Schüler, die ohne Hauptschulabschluss von der Schule abgehen, deutlich zurück, und gleichzeitig stieg der Anteil ausländischer Schülerinnen und Schüler, die die Schule mit einem Realschulabschluss oder mit der Hochschulreife verlieBen (vgl. BMBF 2005).

Die Integrations- und Legitimationsfunktion der Schule thematisiert den Zusammenhang zwischen Schule und dem politischen System. Über die Integrationsfunktion sollen die Heranwachsenden zur Übernahme und Anerkennung der das Zusammenleben in der Gesellschaft (insbesondere im Staat) regelnden Werte und Normen geführt werden. Eine Unterweisung findet eher indirekt statt, auch wenn in den Fächern Politik und Geschichte, z.B. das notwendige Wissen über Staatsverfassung, Rechtsordnung und politische Phänomene vermittelt wird. Wesentlich bedeutsamer ist hier Schule als "Lernfeld der Demokratie", z.B. durch die Mitbestimmung der Schülerinnen und Schüler und die Mitverantwortung in den Einrichtungen der Schulverwaltung oder durch eine demokratische Unterrichtskultur, die auf einer prinzipiellen Gleichwertigkeit von Lehrer und Schüler fußt. . Durch die Leistungen im Bereich der Integrationsfunktion wird erwartet, dass die heranwachsenden Generation die staatlich-politische Ordnung, in unserem Fall die Demokratie und das wirtschaftliche System, die Marktwirtschaft als gerecht und zweckmäßig ansieht und diese dadurch erhalten bleiben. Dies bezeichnet man als (Legitimationsfunktion der Schule).

Lehrerinnen und Lehrer, egal welche Fächer sie unterrichten, bewegen sich in Ihrem schulischen Tun innerhalb dieser Funktionen. Indem sie Schülerinnen und Schüler unterrichten, versuchen sie zu deren Qualifikationen beizutragen, wenn Sie Noten verteilen, handeln sie im Sinn der Selektions- und Allokationsfunktion und durch ihr Verhalten in der Klasse tragen sie - hoffentlich - dazu bei, die demokratische Ordnung unserer Gesellschaft zu legitimieren. 
Bis hierhin lässt sich nun bezüglich des Könnens von Lehrerinnen und Lehrern festhalten, dass dieses so strukturiert sein muss, dass es die Funktionen von Schule erfüllen kann. Konkret bedeutet dies, dass Lehrerinnen und Lehrer in der Lage sein müssen,

- ihre Schüler auf das Leben vorzubereiten

- $\quad$ über die Fähigkeiten der Schüler gerecht zu urteilen.

Diese sollen eine für sie geeignete Schullaufbahn einschlagen.

- $\quad$ ihren Schülern die Werte eines demokratischen Systems zu vermitteln.

Zugegebener Maßen sind das noch keine allzu spezifischen Fähigkeiten für das Können der Lehrerinnen und Lehrer.

Wenden wir uns nun meiner zweiten These zu:

\section{Das Können der Lehrer wird von den Charakteristika und Aufgaben des Lehrberufs determiniert.}

Beschreibt man den Lehrberuf im Unterschied zu anderen Berufen, dann lassen sich folgende Charakteristika feststellen (Ulich 1995, Krause u.a. 2008):

\section{Der Lehrberuf ist ein sicherer Beruf}

Lehrerinnen und Lehrer an den öffentlichen Schulen sind in der Regel Beamte auf Lebenszeit im Dienst der Länder und damit unkündbar. Lehrkräfte an den GrundHaupt- real- und Sonderschulen werden der Laufbahngruppe der gehobenen, Lehrkräfte an Gymnasien und beruflichen Schulen der Gruppe des hohen Dienstes zugeordnet. Angestellte Lehrerinnen und Lehrer stellen die Ausnahme dar .Der Beamtenstatus sichert Unabhängigkeit von Einzelinteressen, er bedeutet jedoch auch Abhängigkeit vom Dienstherren und damit auch von der Öffentlichkeit.

\section{Der Lehrberuf ist ein überalteter Beruf}

Das Durchschnittsalter der Lehrer in Deutschland liegt derzeit bei 47 Jahren und damit ist der deutsche Lehrer im internationalen Vergleich relativ alt. Problematisch ist dabei aber nicht unbedingt die „Überalterung“ der Kollegien in den Schulen, sondern die Tatsache, dass ein großer Teil der Kollegien in den Schulen sich im gleichen fortgeschrittenen Alter befindet. Damit wächst nicht nur die generationenspezifische Distanz zu den Schülern, sondern auch die in jedem Beruf mit fortschreitendem Alter verbundenen Belastungen, können nicht auf unterschiedliche Altersgruppe verteilt werden, sondern ballen sich in einer Altersklasse (vgl. OECD 2003) 


\section{Der Lehrberuf ist ein bürokratischer Beruf}

Lehrerinnen und Lehrer verbringen einen Teil ihrer Arbeitszeit damit, bürokratische Tätigkeiten auszuführen. Flaake betont, dass Verwaltungsarbeit und die in den Erlassen und Reglementierungen erhaltenen Vorschriften von den Lehrern eher ungeliebte Tätigkeiten darstellen. „Richtlinien, Erlasse, Verordnungen werden als Bevormundung, Entmündigung und Entwertung beruflicher Kompetenz erlebt“" (Flaake 1989, S. 91) und verhindern aus Sicht der Lehrkräfte Eigenständigkeit und eigenverantwortliches Handeln. Dies wird von den Lehrern als deutliche Belastung erlebt.

\section{Der Lehrberuf ist ein weiblicher Beruf}

Der Anteil weiblicher Lehrkräfte an den allgemeinbildenden Schulen in Deutschland betrug im Schuljahr 2005/06 68\%. Allerdings variiert der Anteil weiblicher Lehrkräfte stark in den unterschiedlichen Schulformen (von 85\% in den Grundschulen bis zu 48\% in den Gymnasien), so dass die These von der Feminisierung des Lehrberufs vor allem in den unteren Schulstufen und Schultypen zutrifft. Eine mögliche Erklärung für die immer größer werdende Anzahl von Frauen im Lehrberuf lässt sich vor allem auf die Veränderungen des Berufsfeldes «Lehrer/in» zurückzuführen sei. Dazu gehören die vergleichsweise geringen Aufstiegschancen aber auch die gute Kompatibilität von Berufsausübung und Kinderwunsch. Ersteres sorgt dafür, dass Männer eher andere Berufe wählen, zweiteres, dass Frauen sich gehäuft für den Lehrberuf entscheiden. Die Feminisierung der Lehrberufe ist allerdings ein vielschichtiges und komplexes Phänomen. Ein wichtigstes Ziel wäre hier die Attraktivität der Lehrberufe längerfristig und nachhaltig für beide Geschlechter zu steigern. Mangelnde männliche Vorbilder für Jungen und Mädchen in den ersten Schuljahren sind für die Gleichstellung der Geschlechter ebenso wenig förderlich wie die fehlenden weiblichen Vorbilder auf den höheren Schulstufen (vgl. Ulich 1995).

\section{Der Lehrberuf ist ein belastender Beruf}

Der Lehrberuf geht mit einer Reihe von Belastungen einher, die mittlerweile relativ gut untersucht sind, wobei vor allem die nur schwer zu messenden sichtbaren Erfolge der eigenen Arbeit, den Lehrern zu schaffen machen. „Die Ergebnisse der Arbeit bei Lehrenden schlagen sich nicht in einem sichtbaren Produkt oder einem eindeutig meßbarem Ergebnis nieder, sondern in kaum adäquat objektivierbaren geistigen oder psychischen Prozessen.“ (Flaake 1989, S. 114). Viele Lehrer haben zudem das Gefühl, nie mit ihrer Arbeit fertig zu werden, können nur schlecht abschalten und tragen ihre Probleme aus der Schule in die Familie. Die Beanspruchung als Person wird zudem gesteigert durch das unter Lehrern weit verbreitete Gefühl im Unterricht ständig präsent und topfit sein zu müssen. Inwiefern diese belastenden Momente zu negativen Folgewirkungen, wie Burnout oder Stress 
führen, hängt immer von den konkreten schulischen und individuellen Rahmenbedingungen und Bewältigungsressourcen ab. Derzeit gehen neuere arbeitsmedizinische und -psychologische Studien davon aus, dass bis über ein Drittel der deutschen Lehrkräfte sich in einer gesundheitlichen Situation befindet, „bei der sich physische, psychosomatische und psychische Beschwerden vielfältiger Ausprägung überlagern und zu höchst komplexen Krankheitsbildern verdichten können“ (OECD 2003 S.44).

\section{Der Lehrberuf ist kein angesehener Beruf}

Die gesellschaftliche Wahrnehmung der Lehrerschaft ist durch eine geringe öffentliche Wertschätzung gekennzeichnet. Die regelässig vom Institut für Demoskopie Allensbach durchgeführten Berufsprestige Skalen belegen dies für den Gymnasiallehrerruf. Unter den 18 in der Berufsprestige-Skala 2003 vertretenen Berufen, nahm der Studienrat den 14. Platz ein, nur noch unterboten von Offizieren, Politikern, Gewerkschaftsführern und Buchhändlern. Kein anderer vergleichbarer Beruf hat einen dermaßen starken Imageverlust hinnehmen müssen und wird in der Öffentlichkeit so verkürzt und pauschal dargestellt. So titelte der der Spiegel z.B. 2003 ,überfordert, allein gelassen, ausgebrannt“.

\section{Der Lehrberuf ist ein sozialer Beruf}

Lehrer haben - wie Radtke es nennt - ein besonderes von der Gesellschaft lizenziertes Interventions- und Eingriffsrecht in die Lebenspraxis ihrer Schülerinnen und Schüler und gehören damit zur Gruppe der people processing professions (Radtke 2000). Damit sind jene Berufsgruppen gemeint, deren Vertreter folgenreiche Eingriffe in das Leben von Menschen vornehmen können und die deshalb einer besonderen (Selbst-)Kontrolle unterliegen. Im gegliederten Schulsystem fällen Lehrer vor allem an den Gelenkstellen des Schulwesens, also bei allen Übergängen von einer Schulform in die andere, wichtige Entscheidungen über die Bildungskarrieren ihrer Schülerinnen und Schüler. Deswegen tragen sie eine besondere soziale Verantwortung für ihr Tun.

\section{Der Lehrberuf ist ein akademischer Beruf}

Ein Lehrer muss ein Studium absolvieren, wobei diese in der Regel aus zwei Unterrichtsfächern und einem je nach Bundesland unterschiedlich strukturierten erziehungswissenschaftlichen Begleitstudium besteht. Mit den Bemühungen um eine höhere Professionalität im Lehrberuf ist auch die bisherige Struktur der Lehrerbildung in die Kritik geraten und mittlerweile gilt sie als deutlich revisonsbedürftig. Angemahnt wird dabei eine bessere Verzahnung der drei Ausbildungsabschnitte (Studium- Referendariat - Weiterbildung) eine Ausweitung erziehungswissenschaftlicher und fachdidaktischer Anteile und eine an den Bedürfnissen des 
Lehrberufs orientierte Zuschneidung der fachwissenschaftlichen Ausbildungsteile (vgl. z.B. Gogolin 2002, S.2).

\section{Der Lehrberuf ist ein zu professionalisierender Beruf}

Dem Lehrberuf wird im Gegensatz zu den klassischen Professionen (Arzt, Rechtsanwalt, Professor) lediglich der Status der Semi-Professionalität zugestanden, da Lehrer bei ihrer Berufsausübung z.B. keine vollständige Autonomie über die zu lehrenden Inhalte haben, zugestanden. Die Frage nach der Professionalität des Lehrberufs wurde insbesondere in den 1990ger Jahren intensiv diskutiert. Als wesentliche Merkmale einer Profession gelten 1. die Ausübung derselben auf der Basis wissenschaftlicher Grundlagen, 2. eine lange Spezialausbildung, 3. eine altruistische, klientenbezogene Berufsausübung 4. ein gut organisierter Berufsverband der über die Einhaltung der berufsethischen Grundsätze und die Ausbildung und Ausübung des Berufs wacht sowie 5. die weitgehende Autonomie der Professionsinhaber. In der Regel werden Ärzte, Rechtsanwälte, Kleriker und Professoren zu den klassischen Professionen gerechnet und anderen Berufen anhand dieser Kriterien lediglich der Status der Semi-Professionalität zugestanden. Hierzu gehören Krankenschwestern, Sozialarbeiter und auch Lehrerinnen und Lehrer.

Betrachtet man die spezifischen Merkmale der Lehrerarbeit, rechtfertigt sich dieser semi- professionelle Status durchaus, da einige professionstypischen Merkmale erfüllt werden, andere hingegen nicht. Als für eine Profession „typisch“ gelten kann die lange und spezialisierte Ausbildung der Lehrerinnen und Lehrer durch Studium und Referendariat sowie die fachwissenschaftlich fundierte Berufstätigkeit. Zudem können altruistische Motive, die zur Berufswahl beitragen, bei der Mehrheit der Lehrerinnen und Lehrer angenommen werden, da die Verdienstmöglichkeiten für Akademiker außerhalb staatlicher Organisationen deutlich höher liegen. Gegen den Professionsstatus des Lehrberufs sprechen, dass die Berufsverbände weder die Ausbildung, noch die Ausübung des Berufs kontrollieren, da diese Aufgaben von staatlichen Organisationen wahrgenommen werden und Lehrerinnen und Lehrer zudem über keine allzu große Autonomie verfügen, sondern als Beamte weisungsgebunden sind. Im Gegensatz zu anderen Professionen bildete sich auch kein für alle Lehrämter gleichermaßen gültiges Berufsethos heraus, das gleichsam als Gegengewicht zur Autonomie verhindert, dass Privilegien missbraucht werden und auf der Bindung an überpersönliche Werte beruht. Bauer/Kopka/Brindt sehen in diesem Kontext die Ausbildung eines beruflichen Selbst, das allen anderen Komponenten der Professionalität übergeordnet ist und eine strukturierende und integrierende Funktion besitzt, als wichtiges Merkmal der Professionalität von Lehrerinnen und Lehrer an (vgl. Bauer/Kopka/Brindt 1996). 


\section{Der Lehrberuf ist ein didaktischer Beruf}

Mittlerweile wieder stärker in den Fokus gerückt wird die Forderung, den Lehrberuf vor allem als didaktischen Beruf wahrzunehmen und die ,gezielte Planung, Organisation, Gestaltung und Reflexion von Lehr-Lern-Prozessen als den Kernbereich der Aufgaben von Lehrern“ zu betrachten. Die Aufgaben, das Wissen und Können eines Lehrers werden so von der Qualität des Unterrichts her bestimmt. Diese Forderung wendet sich gegen die Ansicht, Lehrerinnen und Lehrer könnten ihre beruflichen Aufgaben allein auf der Basis ihrer Persönlichkeit ausüben. Frühe Veröffentlichungen zum Lehrberuf aus den 1920er und 1930er Jahren des letzten Jahrhunderts z.B. überhöhten persönliche Merkmale des Lehrers normativ und betrachteten sie als einzige Grundlage für gelungene Bildungsprozesse. Kerschensteiner etwa idealisiert den Lehrer als „eine im Dienste der Gemeinschaft stehende Lebensform (...) die aus reiner Neigung zum werdenden unmündigen Menschen (...) dessen seelische Gestaltung nach Maßgabe seiner besonderen Bildsamkeit in dauernder Bestimmtheit zu beeinflussen imstande ist und in der Bestätigung dieser Neigung ihre höchste Befriedigung findet" (Kerschensteiner 1930, S. 46f). Spätere Studien zur Lehrerpersönlichkeit zielten demgegenüber eher auf die Identifikation überindividueller Lehrertypen, wobei insbesondere die Vorstellungen Caselmanns vom logotrophen, der Wissenschaft zugeneigten Lehrer und vom paidotrophen, dem Schüler zugewandten Lehrer (vgl. Caselmann 1949) scheinbar unausrottbar ist. Diese naiven Vorstellungen vom ,guten“ Lehrer gelten mittlerweile als überholt, u.a. auch da sich die Wirksamkeit persönlicher Merkmale eines Lehrers auf die Kompetenzen der Schüler nicht nachweisen lässt (vgl. Helmke 2003, S. ).

In der aktuellen Diskussion um die Kompetenzen von Lehrern wird daher vor allem auf die Entwicklung pädagogischer Professionalität abgehoben. Hierzu gehören berufsspezifische Werte, ebenso wie ein existierendes Handlungsrepertoire zur Bewältigung von Arbeitsaufgaben, die Fähigkeit, sich mit Kollegen in einer nicht-alltäglichen Fachsprache unterhalten zu können, den Bezug auf eine Berufswissenschaft und die Ausbildung eines beruflichen Selbst (vgl. Bauer/Kopka/Brindt 1996). Dieses Selbst stellt den Zusammenhang zwischen Erfahrung, Diagnosekompetenz, Handlungsrepertoire und pädagogischen Werten und Zielen her und ist somit eine Instanz, die „die Aufmerksamkeit eines Pädagogen so steuert, dass Informationen verarbeitet und Handlungsmuster ausgewählt werden, die im Hinblick auf pädagogische Ziele relevant sind“" (Bauer/Kopka/Brindt 1996, S. 14) und die den Arbeitsalltag von Lehrerinnen und Lehrern erleichtern.

Doch welches sind nun die konkreten Aufgaben des Lehrberufs?

Nach der klassischen Definition des Bildungsrates von 1970 bestehen die Aufgaben des Lehrers aus:

Lehren: Hierzu gehört es nicht nur Kenntnisse und Fertigkeiten zu vermitteln, sondern auch das Verständnis für Zusammenhänge zu wecken und Problembewusstsein zu fördern. 
Erziehen: womit die Hilfe zur persönlichen Entfaltung und zur Selbstbestimmung des Schülers gemeint ist.

Beurteilen: Dies meint, die Feststellung der Leistungen genauso wie eine fördernde Diagnose der individuellen Entwicklungs- und Entfaltungsmöglichkeiten.

Beraten: hinsichtlich der persönlichen Schullaufbahn aber auch bei Verhaltensschwierigkeiten

Innovieren: womit gemeint ist, dass Schulentwicklung zur Sache der Lehrer wird, die dazu beitragen sollen Reformkonzepte an den Schulen umsetzten.

Setzt man diese Aufgabenskala mit den bereits erwähnten Charakteristika des Lehrberufs in Verbindung werden die Paradoxien des Lehreralltags und die hiermit einhergehend Überfrachtung des Lehrberufs deutlich. Wie soll ein Lehrer einem Kind dabei helfen, sich persönlich zu entfalten, wenn er es gleichzeitig beurteilen muss und ihm damit u.a. Lebenschancen verbaut? Lehrende sollen Handlungsspielräume nutzen und sich doch an die Verwaltungsvorschriften halten, sie sollen Schülerinteressen berücksichtigen und den Lehrplan erfüllen etc..

Von daher ist es aus meiner Sicht notwendig und durchaus auch entlastend, wenn im nationalen Hintergrundbericht zur Ausbildung, Einstellung und Förderung von Lehrerinnen und Lehrern festgestellt wird,

„dass es nicht die Aufgabe der Schulen und Lehrkeäfte sein kann, Eriiehungsrechte und -pflichten der Eltern zu übernehmen oder problematische Entwicklungen und Defizite zu beheben, die andernorts gesellschaftlich-kulturell verursacht wurden. Die Lehrkräfte müssen zwar für derartige Fragen sensibilisiert werden, und ggf. in der Lage sein, Kontakte zu entsprechenden Fachleuten zu knüpfen: das Zentrum der Lehrertätigkeit bleibt jedoch die Organisation von Lernprozessen. "(OECD 2003, S.30)

Mit dieser Fokussierung des Lehrerhandelns auf den Unterricht ist die Gültigkeit der vom Bildungsrat formulierten Aufgaben nicht generell aufgelöst, sondern sie bekommen eine neue Wertigkeit: Zunächst muss guter Unterricht gemacht werden, dann kommen die anderen Aufgaben hinzu. Dies stellt auch keine Vereinfachung des Lehrerberufs dar, wie man vielleicht meinen könnte, vielmehr müssen Lehrer in die Lage versetzt werden, guten Unterricht geben zu können.

Legt man die Charakteristika und Aufgaben des Lehrberufs als Meßlatte für das Können von Lehrerinnen und Lehrer an, dann scheinen mir folgende Feststellungen besonders wichtig: Das Können der Lehrerinnen und Lehrer: darf nicht mit gesellschaftlich verursachten Problemen überfrachtet werden,

wird in Orientierung an den Standards der jeweilig einschlägigen Wissenschaften erworben und vermittelt, muss zur Kompetenzentwicklung der Schülerinnen und Schüler eingesetzt werden und wird im Unterricht vermittelt. Nun die dritte These: 


\section{Das Können der Lehrer muss auf „guten Unterricht“ zie- len.}

Die Frage, was „guten Unterricht“ ausmacht, ist eine zutiefst normative, und je nach Standpunkt fallen die Aussagen unterschiedlich aus. Befragt man Schülerinnen und Schüler nach ihrer Sicht der Dinge, dann ist Unterricht ,gut", wenn er Spaß macht, sie gute Noten bekommen, ihre Leistungen besser werden, der Lehrer gerecht ist, die Aufgaben verstanden werden, sie sich beteiligen können und der Lehrer hilft, wenn etwas nicht verstanden wurde (vgl. Ditton 2002, S. 281). Die Lehrerinnen und Lehrer wiederum setzen andere Prioritäten. Ein aus Lehrersicht gelungener Unterricht zeichnet sich durch eine geschickte Unterrichtsgestaltung und Wissensvermittlung, eine persönliche Atmosphäre im Unterricht und ein gutes Verhältnis zu den Schülerinnen und Schülern aus (vgl. Terhart u.a. 1994, S. 115-124). Während die Schülerinnen und Schüler also etwas lernen möchten und der Lehrer ihnen dies vermitteln soll, ist aus Lehrersicht das Klima im Unterricht wichtig, damit Schüler gut lernen.

Innerhalb der psychologisch orientierten Lehr-Lernforschung erfolgt die Definition dessen, was guten Unterricht ausmacht, über empirisch fundierte Merkmale. Die Münchner Scholastik-Studie bestimmt Unterrichtsqualität z.B. durch die Dimensionen Klassenführung, Strukturiertheit, Unterstützung, Förderorientierung, soziales Klima und Variabilität der Unterrichtsformen (vgl. Helmke/Weinert 1997, S. 242f). Roßbach, der in seiner Untersuchung zur Unterrichtsqualität im 2. Schuljahr mit ähnlichen Dimensionen arbeitet, unterscheidet zwischen der Prozessqualität, mit der Interaktionen im Unterricht betrachtet werden, der Strukturqualität, mit der die Rahmenbedingungen des Unterrichtens gemeint sind (z.B. Unterrichtszeit, Klassenkontext, räumliche und materielle Ausstattung) und der Qualität der pädagogischen Orientierungen, mit der die Überzeugungen und Werte der Lehrerin untersucht werden (vgl. Roßbach 2002). Ein wichtiger Befund liegt dabei darin, dass bei Qualitätsprozessen manche Faktoren eine größere Rolle spielen als andere. Helmke kommt in seiner zusammenfassenden Einschätzung zur Unterrichtsqualität zu dem Schluss, dass die Effektivität des Unterrichts vor allem durch eine vorhandene fachwissenschaftliche, diagnostische und didaktische Expertise sowie eine stringente Klassenführung gesteigert werden kann, wohingegen eher ,weichere“ Faktoren wie die Gestaltung der Lernumgebung oder die Einstellungen und die Persönlichkeit der Lehrer kaum Effekte zeigen (vgl. Helmke 2003, S. 49ff).

Dies bedeutet nicht, dass die eben genannten „weichen“ Faktoren völlig unwichtig sind für das Klassen- und Schulklima (vgl. z.B. Eder/Gehmacher/Kroismayr 2006, Combe/Helsper 1994) und das Lehrer-SchülerVerhältnis (vgl. z.B. Kanders/Rösner/Rolff 1996, Randoll 1995). Sie lassen sich jedoch nicht kausal belegen. Die von Meyer formulierten zehn Gütekriterien eines „guten Unterrichts“, die er selbst als „theoretisch begründete und in Kenntnis empirischer Forschungsergebnisse formulierte Maßstäbe zur Unterrichtsqualität“ 
(Meyer 2004, S. 20) bezeichnet, können vor diesem Hintergrund als eine Mischung aus empirisch relevanten Merkmalen von Unterrichtsqualität und pädagogisch erwünschten Rahmenbedingungen bewertet werden.

Nach Meyer sind folgende Merkmale für guten Unterricht relevant (Meyer 2004):

Klare Strukturierung des Unterrichts (Prozess-, Ziel- und Inhaltsklarheit; Rollenklarheit, Absprache von Regeln, Ritualen und Freiräumen)

Hoher Anteil echter Lernzeit (durch gutes Zeitmanagement, Pünktlichkeit; Auslagerung von Organisationskram; Rhythmisierung des Tagesablaufs)

Lernförderliches Klima (durch gegenseitigen Respekt, verlässlich eingehaltene Regeln, Verantwortungsübernahme, Gerechtigkeit und Fürsorge)

Methodenvielfalt (Reichtum an Inszenierungstechniken; Vielfalt der Handlungsmuster; Variabilität der Verlaufsformen und Ausbalancierung der methodischen Großformen)

Intelligentes Üben (durch Bewusstmachen von Lernstrategien, Passgenauigkeit der Übungsaufgaben, methodische Variation und Anwendungsbezüge)

Klare Leistungserwartungen (durch Passung und Transparenz) und klare Rückmeldungen (gerecht und zügig)

Zur Umsetzung dieser Anforderung besonders wichtig sind laut Helmke drei basale Expertisen von Lehrerinnen und Lehrern: Die fachwissenschaftliche Expertise umfasst Aspekte der fachlichen Kompetenz eines Lehrers. Sie führt dazu, dass die Lehrperson die zu vermittelnden Lerninhalte sowohl in ihrem wissenschaftlichen Gehalt als auch in ihrer didaktischen Strukturierbarkeit beherrscht. Bestandteile dieser Expertise sind neben dem Fachwissen auch pädagogischpsychologische Kenntnisse darüber, wie sich die Lerninhalte in Bezug auf unterschiedliche Lerngruppen am besten vermitteln lassen. Eine vorhandene didaktische Expertise ist gekennzeichnet durch eine Klarheit der Aufgabenstellung, eine Strukturierung und Überwachung des Lernverlaufs und die Verständlichkeit der Arbeitsaufträge. Sie ist notwendig, um der Vielfalt von Lern- und Leistungsunterschieden in der Klasse und unterschiedlichen Lernzielen gerecht zu werden und zeichnet sich durch „das Gewusst-Wann (welche Unterrichtsziele und curricularen Inhalte eignen sich für welche Unterrichtsmethoden?) und das Gewusst-Für-Wen (welche Schülergruppe profitiert von einer bestimmten Unterrichtsmethode oder leidet unter ihr)“ aus (Helmke 2003, S. 65).

Die Diagnostische Expertise trägt der Tatsache Rechung, dass im Kontext von Schule und Unterricht zahlreiche diagnostische Aufgaben anfallen, die mit ganz unterschiedlichen Zielsetzungen verbunden sind. Hier weiß man mittlerweile, dass die entsprechenden Lerneffekte bei den Schülern dann besonders hoch sind, wenn die Lehrkräfte über eine hohe diagnostische Kompetenz verfügen und ihren Schülern auf dieser Basis gezielte Strukturierungshilfen anbieten können (vgl. Schrader/Helmke 1987, auch Schrader/Helmke 2001). 
Ich formuliere deswegen für die dritte These, dass das Können der Lehrer dazu dient eine fachwissenschaftliche, didaktische und diagnostische Expertise abgeben zu können.

Nachdem ich nun die von mir aufgeworfenen drei Thesen für Sie hoffentlich befriedigend dargestellt habe, möchte ich nun zu der eingangs erwähnten Frage kommen.

\section{Was sollen Lehrer für islamischen Religionsunterricht können?}

Ich will auch dies anhand von 6 Forderungen zusammenfassen und beziehe mich dabei auf meine bisherigen Ausführungen:

\section{Lehrer für Islamischen Religionsunterricht müssen in der Lage sein, die von mir erwähnten} Funktionen von Schule zu erfüllen.

Dies bedeutet, dass sie ihre Schülerinnen und Schüler durch ihren Unterricht befähigen müssen, am gesellschaftlichen Leben in Deutschland teilzuhaben. Hierzu sollten Sie Ihnen im Rahmen ihres Faches -,,Islamischer Religionsunterricht“ religiöse Kompetenzen vermitteln. Was ich damit meine, werde ich gleich erläutern.

\section{Lehrer für Islamischen Religionsunterricht müssen im Rabmen der Selektionsfunktion von Schule, ihre Schüler beurteilen.}

Damit sie dies gerecht tun können, müssen sie über eine diagnostische Expertise verfügen. Auch hierzu werde ich mich später noch äußern.

\section{Lehrer für Islamischen Religionsunterricht sollten dazu beitragen, dass ihre Schüler die demo-} kratische Grundordnung als legitim anerkennen und bereit sind sich in diese zu integrieren.

Dies gelingt allerdings nur, wenn die Lehrerinnen und Lehrer selbst integraler Bestandteil des Schulsystems sind. Sie sollten deswegen formal die gleichen Qualifikationen aufweisen wie andere Lehrerinnen und Lehrer auch, also ein Studium in mindestens zwei Fächern absolviert, ein Staatsexamen und ein Referendariat abgelegt haben. Ich betone dies deswegen so deutlich, da wir eine Situation wie die der Lehrer für muttersprachlichen Ergänzungsunterricht vermeiden sollten. Diese sind einer bayerischen Studie zufolge, nicht in den regulären Schulbetrieb eingebunden, haben kaum Möglichkeiten zur Kommunikation mit Kollegen und werden deswegen von diesen mit ihrer Arbeit auch nicht wahrgenommen (Unbehaun/Schüler 1998). Selbstverständlich sollten die Lehrerinnen und Lehrer für islamischen Religionsunterricht auch Beamte sein, denn nur dies sichert ihnen die 
für den Beruf notwendige Autonomie gegen eine Reglementierung ihres Unterrichts von Seiten des Staates oder religiöser Gemeinschaften.

\section{Lehrer für islamischen Religionsunterricht sollten professionell agieren können.}

Sie können nicht einfach so Lehrerin oder Lehrer werden, weil sie z.B. gläubige Muslime sind, weil sie zufällig bereits andere Aufgaben in einer Schule übernommen und noch einige Stunden frei haben, weil sie von jemand bezahlt werden oder weil sie über eine ideale Persönlichkeit verfügen. Ziel professionellen Handelns ist die Entwicklung eines professionellen Selbst, das dem Lehrer dabei helfen kann, zu entscheiden, wie viel Staat und wie viel Glauben in ihrem Fach notwendig ist, um guten Unterricht zu halten.

Von daher lautet meine fünfte Forderung:

\section{Lehrer für islamischen Religionsunterricht sollten in der Lage sein „guten Unterricht" zu balten.}

Die hierfür notwendigen Kompetenzen müssen sie sich in einem ordentlichen Studium erwerben. Insofern plädiere ich für einen „normalen“ Lehramtstudiengang „Islamischer Religionsunterricht“, mit eigenen Lehrstühlen und eigener Forschung. Die derzeit praktizierten Modelle des Ergänzungsstudiums wie z.B. in Münster mögen für den Übergang taugen, sollten aber keine Orientierung für die Zukunft sein.

Aus einem in Zukunft auszubauenden ordentlichen Studienfach „Islamischer Religionsunterricht" ergeben sich inhaltlich fachwissenschaftliche, fachdidaktische und erziehungswissenschaftliche Anforderungen:

Die erziehungswissenschaftliche Seite scheint hierbei die einfachste zu sein, denn Lehramtstudierende für Islamischen Religionsunterricht müssten in dieser Disziplin die gleichen Anforderungen erfüllen wie alle anderen Lehramtstudierende auch. Ich möchte allerdings betonen, dass die Erziehungswissenschaft keine Hilfswissenschaft für die Fachwissenschaften ist, sondern Lehrern wichtige theoretische Impulse für ihren zukünftigen Beruf liefert.

Hinsichtlich der fachdidaktischen Ausbildung gilt es zunächst Studieninhalte für den Islamischen Religionsunterricht zu entwickeln. Sicher kann hier zunächst eine Anlehnung an allgemeine religionsdidaktische Inhalte erfolgen kann, die dann allerdings von einem Lehrstuhl für islamische Fachdidaktik auf die spezifische Situation im islamischen Religionsunterricht zu übertragen wären.

Was die fachwissenschaftliche Seite angeht, so scheint dies aus meiner Sicht momentan am klärungsbedürftigsten, zu sein, zumindest was das Verhältnis von theologischen, historischen und sprachlichen Anteilen ausmacht. Derzeit scheinen mir die bisherigen Vorschriften, Curricula etc. etwas ,geschichtslastig“ zu sein und hier wäre es wünschenswert auf Dauer zu einem ausgewogenen Verhältnis zu kommen. Allerdings - und das möchte ich hier nochmals betonen - der Bezugspunkt aller Bemühungen müssen die jeweiligen wissenschaftlichen Standards der 
beteiligten religionswissenschaftlichen Teildisziplinen sein, nicht der Glaube an sich.

Ich komme nun nochmals auf die in der ersten Forderung angesprochenen Kompetenzen zurück:

\section{Lehrer für islamischen Religionsunterricht sollen durch ibren Unterricht die Kompetenzen ihrer Schüler erweitern.}

Hiermit ist ein Problem formuliert, dass alle mit der Lehrerbildung betrauten Fächer derzeit haben: Nämlich die Frage: Welche Kompetenzen sollen Schülerinnen und Schüler eigentlich durch das Fach erwerben?

Im Hinblick auf den Religionsunterricht - nicht nur islamischer, sondern jeglicher Art, ist die Annahme, dass es dort darum geht, das „Glauben“ zu lernen oder vorwiegend zu „Beten“, weit verbreitet, aber aus meiner Sicht völlig verkürzt und naiv. Auch wenn ich nun den sicheren Boden der Erziehungswissenschaft verlasse und mich auf das für mich unsichere Terrain der Religionspädagogik begebe, sollte der Religionsunterricht nicht diesen beiden Zwecken allein dienen, sondern Kompetenzen vermitteln, die weit über diese hinausgehen. Rothgangel (2004) betont, dass in einem guten Religionsunterricht Kompetenzen erworben werden,

- die Schülerinnen und Schüler befähigen, eine sachliche Kommunikation über religiöse und gesellschaftliche Schlüsselprobleme zu führen,

- die deren Wissen über religiöse Inhalte erweitern und

- die ihnen Einblicke in und Erfahrung mit religiöser Praxis ermöglichen.

Ich halte dies für eine relativ sinnvolle und empirisch überprüfbare Definition von religiösen Kompetenzen. Die Aufgabe für alle Religionslehrer und damit auch für die Lehrerinnen und Lehrer für islamischen Religionsunterricht bestünde hier darin, diese Kompetenzen zu vermitteln.

Und so lässt sich abschließend die Frage, was Lehrerinnen und Lehrer für islamischen Religionsunterricht können sollen, beantworten mit: Das Gleiche wie andere Lehrerinnen und Lehrer auch. Sie sollten fachlich kompetent sein, Lernleistungen diagnostizieren und Schülerinnen und Schüler in ihrem Lernen unterstützen können.

Wenn Ihnen diese Antwort zu nüchtern ist und sie eigentlich meinen, dass ein Religionslehrer allgemein und ein Lehrer für islamischen Religionsunterricht im Besonderen, doch auch selbst glauben sollte, dann stimmt dies und es stimmt auch wieder nicht. Genauso wie man von einem Sportlehrer erwarten kann, dass er sportlich ist, sollte man auch von einem Religionslehrer erwarten, dass er gläubig ist. Empirisch ist es allerdings nahezu egal, was ein Lehrer glaubt, aber nicht, was ein Lehrer kann. 


\section{Literatur}

Bauer, K. O./ Kopka, A./ Brindt, S.: Pädagogische Professionalität und Lehrerarbeit. Eine qualitativ empirische Studie über professionelles Handeln und Bewusstsein. Weinheim/München 1996

Bellenberg, Gabriele / Klemm, Klaus (1995): Bildungsexpansion und Bildungsbeteiligung. in: Böttcher, Wolfgang / Klemm, Klaus (Hg.): Bildung in Zahlen: Statistisches Handbuch zu Daten und Trends im Bildungsbereich. Weinheim, München, Juventa Verlag, S.217-226.

BMBF Bundesministerium für Bildung und Forschung: Grund- und Strukturdaten 2005. Bonn, Berlin 2005c. Abrufbar unter http://www.bmbf.de/pub/GuS_2005_ges_de.pdf

Caselmann, C.: Wesensformen des Lehrers. Stuttgart 1949

Combe, A./ Helsper, W.: Was geschieht im Klassenzimmer? Perspektiven einer hermeneutischen Schul- und Unterrichtsforschung. Zur Konzeptualisierung der Pädagogik als Handlungstheorie. Weinheim 1994

Ditton, H.: Lehrkräfte und Unterricht aus Schülersicht. Ergebnisse einer Untersuchung im Fach Mathematik. In: Zeitschrift für Pädagogik, 48 (2002) 2, S. 262286

Eder/Gehmacher/Kroismayr 2006,

Fend, Helmut (1980): Theorie der Schule. München, Wien, Baltimore.

Flaake, K.: Berufliche Orientierungen von Lehrerinnen und Lehrern. Eine empirische Untersuchung. Frankfurt/Main 1989

Gogolin, I.: Interkulturelle Bildungsforschung. In: Tippelt, R. (Hrsg.): Handbuch Bildungsforschung. Opladen 2002, S. 263-279

Gudjons, H.: Pädagogisches Grundwissen. 8. Auflage, Bad Heilbrunn 2003

Helmke, A.: Unterrichtsqualität erfassen, bewerten, verbessern. 4. Aufl. Seelze 2003

Helmke, A./ Weinert, F. E.: Bedingungsfaktoren schulischer Leistungen. In: Weinert, F. E. (Hrsg.): Psychologie des Unterrichts und der Schule. Göttingen 1997 
Kanders, M./ Rösner, E./ Rolff, H.-G.: Das Bild der Schule aus der Sicht von Schülern und Lehrern. Ergebnisse zweier IFS-Repräsentativbefragungen. In: Jahrbuch der Schulentwicklung (1996) 9, S. 57-113

Kerschensteiner, G. M.: Die Seele des Erziehers und das Problem der Lehrerbildung. 3. Auflage, Leipzig 1930

Krause, Andreas; Schüpbach, Heinz; Ulich, Eberhard; Wülser, Marc Titel: Arbeitsort Schule.

Organisations- und arbeitspsychologische Perspektiven. Quelle: Wiesbaden: Gabler (2008),

Meyer, H.: Was ist guter Unterricht? 2. Auflage, Berlin 2004

OECD 2003: Nationaler Hintergrundbericht. Online abrufbar unter: http://www.oecd.org/dataoecd/56/38/31076222.pdf:

OECD Education at a glance

Radke, F.O.: Professionalisierung der Lehrerbildung durch Autonomisierung, Entstaatlichung, Modularisierung. In: sowi-onlinejournal0/2000. Abrufbar unter http://www.sowi-onlinejournal.de/lehrerbildung/radtke.htm

Randoll, D.: Schule im Urteil von Lehrern. Ergebnisse einer Befragung von Oberstufenlehrern aus vier alten und einem neuen Bundesland zu ihrer Wahrnehmung von Schule. Göttingen 1995

Rossbach, H.-G.: Unterrichtsqualität im 2. Schuljahr - Ergebnisse einer empirischen Untersuchung. In: Unterrichtswissenschaft, 30 (2002) 3, S. 230-245

Qualitätskriterien "guten" Religionsunterrichts, in: Rothgangel, M.; Fischer, D. (Hrsg.), Standards für religiöse Bildung? Zur Reformdiskussion in Schule und Lehrerbildung, Münster 2004, 104-118.

Schrader, F.-W./ Helmke, A.: Alltägliche Leistungsbeurteilung durch Lehrer. In: Weinert, F. E. (Hrsg.): Leistungsmessungen in Schulen. Weinheim 2001, S. 45-58

Schrader, F.-W./ Helmke, A.: Diagnostische Kompetenz von Lehrern: Komponenten und Wirkungen. In: Empirische Pädagogik,1 (1987) 1, S. 27-52

Terhart, E./ Czerwenka, K./ Ehrich, K./ Jordan, F./ Schmidt, H.-J.: Berufsbiographien von Lehrern und Lehrerinnen. Frankfurt/Main 1994

Tillmann, Klaus-Jürgen: Viel Selektion - wenig Leistung. Ein empirischer Blick auf Erfolg und Scheitern in deutschen Schulen.In: Liebau, Eckart / Zirfas, Jörg: Ungerechtigkeit der Bildung - Bildung der Ungerechtigkeit. Opladen u.a.: Budrich (2008), S. 155-173

Ulich,. K. Beruf Lehrer/in. Arbeitsbelastungen, Beziehungskonflikte, Zufriedenheit. Beltz: Weinheim/Basel 1996

Unbehaun, H./ Schüler, H.: Türkische Lehrer in Bayern. Bamberg 1998. Abrufbar unter http://www.opus-bayern.de/uni-

bamberg/volltexte/2005/10/pdf/Tubayonline.pdf [23.11.2009] 

Bemerkungen zum Schluss 


\section{Betram Schmitz, Kinan Darwisch: Zwischen Würdigung und Verantwortung - Perspektiven zum Islamunterricht an deutschen Schulen}

\section{Verheißungsvolle Anfänge}

„Machen Sie irgendetwas, aber machen Sie was. - Es muss losgehen. Egal wie.“ Als Dozent im Osnabrücker Versuchsprojekt der Bund-Länder-Kommission zur Ausbildung muslimischer Islamlehrer habe ich diese Äußerung mehrmals gehört. Einladender kann man mit einem Unternehmen nicht empfangen werden - wobei es wie selbstverständlich ein Vertrauen in unsere Arbeit implizierte, denn das „Egal wie“ meint natürlich „,in verantwortungsvoller Weise“. Drei Jahre haben mein Kollege Dr. Bodenstein und ich unter der damaligen pädagogischen Leitung von Professor Peter Graf Lehrerkandidaten für den Islamunterricht ausgebildet. Der eine Schwerpunkt lag darin, die eigenen Kenntnisse über den Islam in theologischer, historischer und sozialer Weise zu vertiefen, der andere, aus religionswissenschaftlicher Sicht den Islam im Kontext der Nachbarreligionen, Judentum und Christentum zu verstehen, ihn in der Innen- und Außenperspektive wahrzunehmen. Wir haben nicht nur mit den Studierenden sehr gute Erfahrungen gemacht, 
sondern haben auch die Bereitschaft gespürt, von Seiten muslimischer Bürger, solche Ausbildungsmöglichkeiten anzunehmen, damit der Islamunterricht an Schulen fachkundig geführt werden kann. Die von Frau Ballasch zitierten positiven Ergebnisse des Schulversuchs zeigen: So schlimm wie befürchtet werden könnte, kann es mit dem Islamunterricht an Schulen eigentlich gar nicht werden. Anfang muss nicht schwer sein, denn es gibt das uneingeschränkte, offenherzige, grundsätzliche Interesse, dass ich als Ausbilder möglicher Islamlehrer in Osnabrück erfahren habe.

Es gibt viele Bücher zum Religionsunterricht insgesamt, gleich welcher Konfession und Religion, psychologische Entwicklungsmodelle über Kinder und Religionsverständnis, engagierte Kinderbücher und viele Lehrer, die schon seit langen Jahren mit Liebe, Kreativität und Geist dabei sind, muslimischen Kindern ihre Religion zu vermitteln. Auch die Islamlehrerausbildung fängt nicht von vorne an, weder in der Türkei noch in anderen Ländern, noch in Deutschland selbst. Unser universitärer Versuch, mögliche Islamlehrer auszubilden ist für die erste Generation schon seit über zwei Jahren abgeschlossen. Die Studierenden waren zufrieden, die Lehrenden ebenfalls. Aber vielleicht liegt an diesem Punkt das eigentliche Problem: Der damalige Leiter, Professor Graf, zitierte mit seinem Interesse für den Buddhismus gerne den klassischen Zenvers des offenen Anfängergeistes. Im Anfang ist noch alles möglich, offen, frisch - wenn der Geist dazu bereit ist. Unsere Ausbildung wähnte sich - vielleicht etwas zu freimütig - in diesem Anfängergeist und schaute auf die Chancen, die in einem Fach bereitstehen, dass noch nicht in allzu feste Bahnen gezwängt und durchnormiert ist.

Die nüchterne Realität begegnet einem nicht im Fach selbst, dem Islam mit seiner Breite, Erhabenheit und Tiefe. Sie begegnet einem noch nicht einmal in den Elternerwartungen, die weitest gehend abwartend offen $\mathrm{zu}$ sein scheinen, auch wenn sich einzelne strenge Blicke unter ihnen finden. Sie begegnen einem schon eher, wenn eine Fraktion der Kopftuchtragenden befürchtet, nicht eingestellt zu werden, weil sie im Unterricht dieses Kopftuch nicht ablegen mag - und der weit größeren Fraktion, die befürchten muss, wenn das Kopftuch einmal gebilligt ist, dass es dann für alle anderen zur unausgesprochenen, aber umso wirksameren Forderung wird und sie sich nicht vorstellen können, weder in der Türkei noch in Deutschland noch im Unterricht eines tragen zu müssen. Ebenso ist die Frage, ob nicht auch eine Muslima ohne Kopftuch eine vollwertige Muslima gar IslamLehrerin, sein kann. Mitunter scheinen sogar, was die Anzahl der Fotographien in Berichten über Geschehnisse im Zusammenhang mit dem Islam oder z. B. christlich-muslimische Begegnungen betrifft, nur Bekopftuchte richtige Muslimas zu sein. Aus den Erfahrungen als Hochschuldozent bin ich gerne bereit, für die von uns ausgebildeten Lehrer und Lehrerinnen einzutreten, für die mit Kopftuch, wie für die ohne ein solches! Leider werden unsere Kursteilnehmer (und evtl. andere in anderen Kursen) trotz ihres 3-jährigen Kurses im probeweisen Ausbildungsgang offensichtlich nicht als „ausgebildet“ verstanden, sonst könnte nicht davon 
gesprochen werden, dass es keine ausgebildeten Lehrer gäbe. An solchen Punkten sehe ich eher die oben angesprochenen Probleme: Wird das, was in den verschiedenen Ausbildungsgängen gemacht wird, anerkannt, oder sprechen sich die Ausbildungsformen gegenseitig ihre Kompetenz ab?

\section{Religionsunterricht im Experiment}

An unserer Studieneinheit in der Sommerakademie in Canakkale ermutigte ich eine Studierende, ihren Standpunkt gegenüber den dortigen Professoren zu artikulieren. Darauf fragte sie (unbedarft) nach einem „liberalen Islam“, worauf sie die viel sagende Antwort erhielt, man sei Muslim oder nicht. Wenn die innere Logik der Antwort nichts zu wünschen übrig lässt, so bleibt auch der dahinter stehende Anspruch nicht verborgen. Ist damit ein Experimentieren unerwünscht, gar unerlaubt? Phasen, die christlichen Religionslehrern, evangelischen wie katholischen, selbstverständlich sind, Experimentiermöglichkeiten, die sie (hoffentlich noch lange) haben, sollen Islamlehrern/lehrerinnen und Ausbildern verschlossen bleiben - gerade in einer Zeit, in der der „Islam in Deutschland“ experimentieren muss, weil er noch nicht auf eine jahrzehntelange etablierte Geschichte zurückblickt?

Selbst überzeugte Muslime scheinen dabei zwischen dem doppelten Anspruch zu stehen, dass ihre Religion von der Umgebung entweder nicht „für voll“37 genommen wird, oder aber, dass sie selbst verteidigen müssen, dass sie die beste, einzige und wahre ${ }^{38}$ Religion gegenüber allen anderen Religionen und erst Recht dem Unglauben ist. Umso mehr könnte diese polare Diskrepanz diejenigen verunsichern, die nicht so tief in ihrem Glauben verwurzelt sind. Die Einrichtung des Schulunterrichts könnte gerade diese Lücke füllen - der Islam wird ernst genommen, er wird unterrichtet (in einer Form, wie sie auch für die so genannte Mehrheitsgesellschaft akzeptabel ist) und damit anerkannt, ebenso wie auf der einen Seite der Katholizismus, auf der anderen Seite der Protestantismus, - oder auch andere Religionen. Es mag dann eher selbstverständlich werden, dass man in

37 Auch von christlich theologischer Seite scheint es oft schwierig, sich auf die Perspektive des Islams ernsthaft einzulassen und dabei einerseits die Diskrepanz zwischen Christentum und Islam in ihrer Tiefe zu erfassen und andererseits die Theologie des Korans und des Islams zu würdigen, auch wenn sie einem fremd ist. Ein Pseudoverständnis hilft an dieser Stelle, so wird deutlich, nicht wirklich weiter, sondern offenbart eher die Untiefen.

38 Damit soll keiner Religion der Anspruch aberkannt werden, sich für „,wahr“ halten zu dürfen genau dieser Anspruch definiert mitunter nach religionsinternen Definitionen „Religion“, als der Glaube an das „Wahre“, etwa als das, was einen (als wahr) unbedingt angeht (vgl. Paul Tillich, ähnlich, nur mit andern Worten, Martin Luther). Eine Relativierungsforderung an Religionen, sich als eine (beliebige) von vielen zu verstehen, käme damit letztlich einer Auflösung des existenziellen Anspruchs nah. Entscheidender ist dabei der Respekt vor dem jeweiligen Anspruch des Anderen, der in einem anderen Kontext lebt, der ihn im Innersten bestimmt. 
Deutschland Jude, Christ, Muslim, Hindu, Buddhist, Bahai ist, oder sonst einer anderen traditionellen Religionsgemeinschaft angehört.

\section{Religion und gesellschaftliche Verantwortung}

Bei den beispielhaft aufgelisteten Religionsgruppen handelt sich um Gemeinschaften, die in gewissem Sinn gereift sind, was sie nicht gegen Polarisierung immun macht, doch gewährleistet, dass sie auch ihren inneren Selbstwiderspruch, ihre Fähigkeit zur Selbstkritik in sich haben. Es sind damit Religionsgemeinschaften gemeint, die im Laufe ihrer Geschichte selbst schon oben waren und unten, die gemäß den sozialen Schichten durchwachsen sind, von Armen und Reichen, Intellektuellen und Praktikern, Reformern und Restaurateuren, plural geworden sind und in sich vielgestaltig und die diesen langen Transformationsprozess überstanden haben. Aus dieser gewachsenen Haltung heraus können sie wiederum ihren Reichtum in die Gesellschaft einbringen - und werden mehr und mehr zu gefragten Teilnehmern, nicht unbedingt, weil sie (etwa aufgrund göttlichen Anspruchs) alles besser wissen, sondern weil sie sich in der Gesellschaft bewährt haben und Teil von ihr sind und so aus ihrer spezifischen Perspektive einen bedenkenswerten Blickwinkel beitragen können.

Dieses Letztgenannte ist ein entscheidender Grund, warum Religion an Schulen gelehrt werden soll, - und er war für die Verankerung im Grundgesetz nicht unerheblich: Jede Institution, sei sie religiös, kulturell, staatspolitisch oder juristisch, ist nicht unfehlbar. Sie bedarf ständig der Möglichkeit eines Korrektivs, das sie nicht zum Schweigen bringen darf!

Das ist eine Lehre, die bei der Einrichtung des Grundgesetzes aus der deutschen Vergangenheit gezogen wurde. Kein Mensch, aber auch keine Partei, Religion oder sonstige Institution darf sich absolut setzen, vielmehr noch, sie darf sich nicht einmal absolut setzten können. So ist sind z. B. auch bewusst die rechtlichen Bereiche von einander getrennt und in der Gewaltenteilung je in sich unabhängig (Legislative, Jurisdiktion, Exekutive). In dieser Weise liegt es nicht nur am Staat, den Religionsgemeinschaften ihren Rahmen zu geben, sondern auch an den Religionsgemeinschaften, sich politisch zu äußern. Die Voraussetzung, damit dies in beide Richtungen gelingt, ist die gegenseitige Würdigung und Wertschätzung.

Dies setzt allerdings voraus, dass „Religion“ nicht von vorn herein im Contraverhältnis zum Staat an den Schulen gelehrt wird, sondern in grundsätzlicher Anerkennung seiner Prinzipien. Erst daraus erwächst ihm die Möglichkeit, den Staat an seinen eigenen Prinzipien zu messen, wie etwa an der Verwirklichung der Würde von Mann und Frau, dem Recht, Einfluss auf die Politik zu nehmen, sich in der Presse frei zu äußern etc. Diese Möglichkeit der Kritik am Staat, an anderen Einrichtungen und ebenso an anderen Religionen setzt wiederum die Befähigung zur Selbstkritik und Selbsthinterfragung voraus. Es ist die eine Sache, dass es erst einer französischen Revolution bedurfte, auch der Kirche zu verdeutlichen, dass es 
keine Geburtsstandesvorrechte geben dürfte. Es ist eine andere Sache, dass das Christentum der Gegenwart diesen Punkt aufs Tiefste verinnerlicht hat und nun als eigenes Anliegen vertritt, ebenso etwa die die Gleichberechtigung der Ehe. Solche Momente sind dem Christentum nicht leichter oder schwerer gefallen, als dies beim Islam der Fall sein müsste! Der Koran gibt beides problemlos her wobei das Standesrecht weniger ein Thema des Islams war.

\section{Der Schulversuch}

Vielleicht zeigt die Evaluation des Schulversuchs noch etwas anderes: Zunächst könnte festgehalten werden, dass allein die Würdigung des Islams als anerkannte Religion etwas positives bewirken und aus einer Verteidigungshaltung ein Gegenüber machen kann. Darüber hinaus könnte sich aber auch die Selbstregulierung eines flexibel-offenen Systems zeigen, das grundsätzlich auf Integration und friedliches Zusammenleben zielt und dies umso mehr, wie es offen und flexibel gehalten und nicht zentral genormt wird.

Unser Bildungssystem basiert und vertraut (eigentlich) stark auf die Fähigkeit, nicht nur Informationsmengen aufzunehmen, sondern kritisch-selbstkritischen Wesen die Möglichkeit zur Entfaltung zu geben, dafür allerdings einen Rahmen bereit zu stellen. Vom Anspruch des Korans her ist der Islam im höchsten Maße eine ethische Religion. Durch seine - zumeist von der Außenwelt überwiegend negativ bewertete - durchgehende Betonung des göttlichen Gerichts muss sie den Glaubenden nicht in eine beständige Lebenssituation der Angst versetzen. Sie kann ihm vielmehr verdeutlichen, dass sein gesamtes Denken, Fühlen und Tun unter den Augen Gottes steht und vor ihm verantwortet werden muss. Das Gericht liegt an ihm selbst, es gibt keinen Ausweg, kein Dunkel, keine Flucht, - aber auch nichts an Guten, Rechten, Hilfsbereitem und Schönen, dass Gott je vergisst. So ist jeder Einzelne für sich bei Gott aufgehoben, im Guten wie im Bösen, es wird ihm kein Unrecht getan - er oder sie kann sich gewiss sein, dass das göttliche Gericht fair vollzogen, vielleicht sogar (durch die göttliche Gnade) mehr als dass. Er hat sich in Aufrichtigkeit (!) für das Gute in der Welt einzusetzen, sie nicht zu vernichten, kein Unheil auf der Welt anzurichten. Wird dieser Anspruch in Würde und ohne Doppelbödigkeit von einem Muslim umgesetzt, wie sollte seine Stimme nicht von der Gemeinschaft um ihn mit Achtung gehört werden. Für den Staat wird diese Stimme seines Bürgers so wichtig sein, wie die einer Person, die sich in der Gnade Gottes angenommen weiß und nun sein Leben aus dieser göttlichen Gnade heraus in christlicher (Nächsten- und Feindes)Liebe zu gestalten aufgerufen ist.

Demgegenüber - so ist es gedacht - verspricht der Staat jedem Bürger, gleich welcher Religion, dass Recht auf körperlicher Unversehrtheit, Gleichberechtigung, Achtung und etwa die freie Wahl des Glaubens, aus dem einzigen Grund heraus, weil diese Person Bürger des Staats ist. Er erwartet dafür, dass dieser Bürger gera- 
de diese Rechte für alle achtet und nicht sein eigenes Recht oder das seiner Glaubensgemeinschaft über das des Einzelnen setzt. So wird erwartet, dass auch im Religionsunterricht der Rahmen dieses Staats und seiner Gesetze geachtet wird, in dem die unterschiedlichen Religionsgemeinschaften ihren Platz eine neben der anderen erhalten sollen, - wohl wissend, dass nicht eine wie die andere ist, sondern jede ihre Charakteristik und Qualität hat. Über diese und ihren Wahrheitsanspruch zu urteilen, ist dann wiederum nicht Angelegenheit des Staats, solange der weltliche Rahmen erfüllt wird. Es ist vielmehr Aufgabe der Selbstkritik dieser Religionsgemeinschaften und der Kritik dieser untereinander, sowie der Anfragen von Außen.

Diese Momente von Kritik, Selbstkritik und Anfrage von Außen sind besonders gefragt, wenn der Islam in die universitäre Ausbildung übergeht - denn diese setzt die genannten Momente geradezu voraus. Auch wenn die Selbstfindung des Islams in Deutschland gerade einen bedeutenden Prozess durchläuft, so sollte diese - besonders an der Universität - doch das kritische Reflexionspotential integrieren und sich nicht auf Tradition und Empirie verlassen. Es ist gerade die Stärke der Theologie - und ein bedeutsamer Grund sie in den Universitätskanon einzuschließen -, dass sie sich damit diesen wissenschaftlichen Forderungen eindeutig verpflichtet, selbst wenn ihr eigentliches Fundament sich auf die Transzendenz beruft. Umso wichtiger ist es, diesem Anspruch nicht blind und unreflektiert zu folgen, sondern sich hinterfragend dessen bewusst zu sein, was man tut - und selbst, was man tut, wenn man glaubt.

\section{Fazit}

Diese das aktuelle Geschehen sowie die damit verbundenen Probleme und Fragen sowie Bedenken -insbesondere seitens der mehrheitsgesellschaftlichen und wissenschaftlichen Seite- im Kontext der Einrichtung eines islamischen Religionsunterrichts und der Integration des Islams hierzulande widerspiegelnden Worte meines religionswissenschaftlichen Kollegen Dr. Dr. Bertram Schmitz machen auf den Punkt gebracht vor allem eines deutlich: Wir brauchen mehr Transparenz, Vertrauen und vor allem ein stärkeres Aufeinanderzugehen, welches losgelöst ist von jeglichem Nischendenken, um bei der Integration der Muslime sowie vor allem der Einrichtung eines ordentlichen islamischen Religionsunterrichts voranzukommen.

Dieses Aufeinanderzugehen sollte aber auch eine von allen Akteuren getragene Zielsetzung aufweisen, nämlich das gemeinsame konstruktive und kooperative Miteinanderleben von Muslimen und Nicht-Muslimen in der Bundesrepublik auf dem Boden des Grundgesetzes.

Die Mehrheitsgesellschaft scheint hierbei partiell immer noch Nachholbedarf im verständnisvollen Umgang mit den Muslimen zu haben, wenngleich vor allem im Kontext der begrüßenswerten Islam-Konferenz Fortschritte gemacht worden 
sind. Es muss dabei verstanden werden, dass Integration nicht mit Assimilation gleichzusetzen ist und auch Unterschiede anerkannt werden müssen. Es sollte zwar kein Zweifel darin bestehen, dass die pluralistischen und säkularen Werte des Grundgesetzes nicht zur Debatte stehen und der pluralistische Geist des Grundgesetzes von jedem Gesellschaftsmitglied respektiert werden muss, aber dennoch ist es von Relevanz, die muslimische Minderheit mit Respekt zu behandeln und Unterschiede anzuerkennen sowie die Muslime als gleichwertige und gleichberechtigte Partner zu sehen. Zu oft gewinnt man noch den Eindruck, dass dies nicht geschieht und man die Muslime bevormunden möchte und sie dementsprechend auch behandelt werden sowie letztlich auch mangelhaftes Wissen über ihre Religion und Kultur in weiten Breiten der Mehrheitsgesellschaft zu existieren scheint.

Auf der anderen Seite ist von muslimischer Seite die Bildung von Religionsgemeinschaften auf Landesebene zu verlangen, vor allem aber endlich eine deutliche, sachkundige und vor allem transparente Bekennung zum Grundgesetz.

Auch müssen sich die muslimischen Verbände eines bewusst sein, beziehungsweise werden: Wenn sie nicht ihren Part erfüllen und der Prozess der Einrichtung eines ordentlichen islamischen Religionsunterrichts und der Integration der Muslime hierzulande generell nur schleppend voran kommt, so sind vor allem die muslimischen Jugendlichen die Leidtragenden und gerade auch die muslimischen Verbände tragen dabei eine Mitverantwortung. Zu gerne scheint von Teilen der muslimischen Community mit dem Finger auf die politische, mediale und kirchliche Ebene gezeigt und diesen die Alleinverantwortung gegeben zu werden. Dies führt aber nicht weiter und zeigt vor allem eines, nämlich dass man noch nicht bereit zu sein scheint als souveräner Ansprechpartner im Sinne einer Religionsgemeinschaft zu fungieren.

Ich hoffe daher, dass sich die muslimischen Vertreter dies zu Herzen nehmen und endlich anfangen in einer nachhaltigen selbstkritischen, aber dennoch selbstbewussten Art und Weise für die Belange der Muslime in Deutschland, beziehungsweise ihrer Mitglieder einzutreten. Dazu bedarf es auch einer Loslösung von politischen und national bedingten Streitigkeiten, bzw. Interessen der hierzulande sesshaften islamischen Dachverbände und der damit verbundenen Agitationsorientierung. Damit liegt letztlich immer noch ein langer Weg vor allen Beteiligten und man scheint das Anfangsstadium dieses Weges immer noch nicht verlassen zu haben. Doch dieser Weg erscheint machbar und alle Seiten können diesen Weg bewältigen, wenn man sich im gegenseitigen Respekt an die Kernthemen wagt und diese konstruktiv sowie kompromissbereit lösen will. 


\section{Firouz Vladi: Was nötig ist: Mit Vertrauen auf Klassenfahrt, interkulturelle Kompetenz für Schulen und Jugendeinrichtungen sowie einige Anmerkungen zu einer korrekten islamischen Terminologie}

\section{Integration statt Assimilation: welche Veränderungen erfährt die aufnehmende Gesellschaft?}

In der Wertedebatte gilt auch weiterhin der verfassungsrechtliche Grundsatz: der Staat lebt von Voraussetzungen, die er nicht selber schaffen kann. Die Grundwerte unserer Verfassung sind nach christlichem Verständnis vorrangig christliche Grundwerte; aber auch Ergebnis der Aufklärung aus den europäischen Befreiungsbewegungen. Diese Werte konnten 1949 erfolgreich in den bundesdeutschen Verfassungsstaat freiheitlich demokratischer Grundordnung übernommen werden. Fünf vom Hundert der heutigen deutschen Bevölkerung, mit steigender Tendenz, stellt der muslimische Bevölkerungsanteil. Die Folge des demografischen Wandels sowie des demokratischen und religionsneutralen Grundkonzeptes: die Mehrheitsgesellschaft muss und wird sich verändern, sich öffnen für andere. Diese 
bringen meist keine neuen und anderen, vielmehr die alten, früher auch hier gepflegten Werte, Sitten und Regeln mit; deutlich wird dies im Stellenwert der gröBeren Familie.

Die Hinwendung des Menschen zu Gott und zum Nächsten ist im Islam als Dualismus ausgelegt. Dazu gehört untrennbar die Verwirklichung von Gerechtigkeit aufgrund der authentischen islamischen Quellen: Qur'an und Sunnah. Das islamische Recht (Scharia) gebiert die Chance für die hiesigen Muslime und verpflichtet sie darauf, die Methoden der islamischen Rechtsfindung für ihr Leben in Europa und neu zu beleben und im Kontext hier verwirklichter Gerechtigkeitsmodelle zu verstehen; wir sehen hierin eine Herausforderung für die islamischwissenschaftlichen Forschung und Lehre in den Universitäten.

Gemeinsam mit gläubigen Christen und Juden möchten muslimische Bürgerinnen und Bürger Deutschlands das Gemeingut der aus der Religion begründeten Werte und Lebensregeln gegen Agnostizismus und Atheismus verteidigen. Solche Strömungen sollten u. E. nicht den kleinsten gemeinsamen Nenner Deutschlands bilden.

\section{Muss sich eine Weltreligion der durchgehend koeduka- tiven Schulpädagogik anschließen?}

Die Koedukation ist eine bürgerliche Errungenschaft. Ging es zunächst darum, Mädchen einen Zugang zu höherer Bildung zu ermöglichen, stehen heute eher Fragen der Bildungsgerechtigkeit und einer geschlechterbewussten Schule im Vordergrund. Neu hinzu kommen - auch im Lichte der verfassungsmäßigen Freiheitsgarantien - religiöse Aspekte der Erziehung, des Verhältnisses der Geschlechter oder Generationen zueinander sowie insbesondere von Sittlichkeit.

Muslime wollen nicht aus Biologie-, Schwimm- und Sportunterricht aussteigen, sie wollen ihre Töchter nicht ertrinken sehen. Über die Zeugung und das Wachsen im Mutterleib hat Gott im Qur'an für alle offenbart; warum sollen Kinder dies in der Schule getrennt wahrnehmen? Muslimische Eltern und Kinder wollen diese Bildungsziele auf bestem Niveau verwirklicht wissen. Es gibt ja bzgl. eines getrennten Schwimmunterrichts schon viele positive Beispiele in großen Städten. Deutschland ist kein armes Land und die Implementation von Phasen getrennten Unterrichts wird hierfür eingefordert; und so sollte aus pädagogischer Sicht sicher nichts dagegen sprechen, die Koedukation punktuell aufzuheben. Für den Sportunterricht kann sittliche Kleidung ohne Schwierigkeiten angenommen werden, ohne Merkmale der Unfallsicherheit in den Vordergrund zu stellen. Entsprechendes gilt für Klassenfahrten, wo ja bis heute die Geschlechtertrennung in Schlaf- und Waschräumen unverändert fortgilt. Der Topos Schwimmunterricht darf nicht länger Teil kollektiver Polemik gegen die muslimischen MitbürgerInnen sein. 


\section{Scheitert Schule, solange Instrumente des Vertrauens Eltern-Schule nicht installiert werden?}

Veröffentlichungen zeigen, dass zugewanderte Familien sich in ihren Wertvorstellungen nicht wesentlich von einheimischen Familien unterscheiden (vgl. SinusStudie 200939). Dennoch kommt es vor allen in schulischen Kontexten oftmals zu „Missverständnissen“ zwischen Migranten und Migrantinnen einerseits und Lehrerinnen und Lehrern andererseits. Dies gilt besonders in Bezug auf muslimische Kinder.

In einer globalisierenden und zunehmend anonymeren Welt werden Vertrauensinstrumente institutionalisiert, um erfolgreich zu bleiben, dies reicht von Qualitätszertifikaten in der Erwachsenenbildung bis zu DIN- und ISO-Normen in der Industrie. Qualitätsmanagement mit Einbeziehung aller Beteiligten sichert den Erfolg, weil der Kunde eine Grundlage für Vertrauen in Qualität, Service oder Umweltkonformität findet. Ebensolche Instrumente könnten auch zu einer diskriminierungsfreien Multikulturalität und -religiosität in der Schule, im Kindergarten, der Jugendherberge oder im Landschulheim beitragen sowie zur Berücksichtigung von religiösen und rituellen Alltagsaspekten im Tagesablauf oder Jahresverlauf von Schülerinnen und Schülern. Es gibt erfolgreich arbeitende Institutionen, die sich auf das Training von Multiplikatoren u.a. spezialisiert haben wie etwa Pro Dialog Köln ${ }^{40}$.

Solche Weiterbildung ist an Inhalte geknüpft. Wie im schulischen Religionsunterricht so muss auch hier sichergestellt werden, dass die Inhalte der Religion von der Religionsgemeinschaft getragen werden. Dies ist hier auch deshalb wichtig, weil in Kindergarten wie Schule muslimische Kinder (und Eltern) der verschiedensten Prägung zusammen kommen: nicht nur Sunniten und Schiiten, vielmehr trifft hier auch die gelebte Religion samt ihrer Orthopraxie auf alle Formen der Säkularisierung. Man muss wissen, dass Migration oft auch Selektion ist, wie wir dies z.B. stark bei iranischen Familien nach 1979 beobachten, die mit dem Verlassen des Landes oft genug auch die Orthopraxie, die gelebten Aspekte der Religion hinter sich lassen. Nun steht es im Erziehungs- und Schulbereich der Kultusverwaltung nicht an, aus diesen unterschiedlichen Tiefen des Glaubens, ausgedrückt in Orthopraxie und religiösem Recht, einen pflegeleichten oder angepassten „Wunsch-Islam“ zum Maßstab zu erklären.

Welche Themen sollten in einem solchen interkulturellen Training in Hinblick auf die Belange der Muslime abgearbeitet werden? Hier nur Stichworte, die an anderer Stelle der Wertung und Vertiefung bedürfen: Gender, Generationen, Speisegebote, Fasten (Oberstufe), Gebetszeiten und -möglichkeiten, Kleidung, Feier-

\footnotetext{
39 Erste Ergebnisse der Studie abrufbar unter:

http://www.sociovision.de/uploads/tx_mpdownloadcenter/MigrantenMilieus_Zentrale_Ergeb nisse_09122008.pdf, Weitere Veröffentlichungen unter http://www1.bpb.de/files/R32I01.pdf 40 www.pro-dialog-koeln.de/dateien/prodialog.php
} 
tage, Schutz der Intimsphäre und vor den Gefahren religiös nicht erlaubter sexueller Beziehungen, Sprache und Begriffe, Abgewöhnen patriarchalischer Missbräuche und Trennen von religiösen Bindungen durch Nichtfürwichtighalten; Nutzenlernen von Informationsquellen (Fähigkeit, Kenntnisse statt Vorurteile jenseits der Medien zu erlangen). Beispiel Fasten: Im Ramadhan werden Kinder allmählich an das reguläre Fasten herangeführt. Sie erleben es als Freude und sind stolz, wenn sie fünf Stunden enthaltsam bleiben konnten. Lehrkräfte aber vermuten elterlichen Zwang und wollen es den Kindern - auch mit Rundschreiben an die Eltern austreiben; so erlebt in einer südniedersächsischen Realschule; wie können sich in einem derart religionsunsensiblen Umfeld Kinder angenommen fühlen? Hierher gehört auch der Rechtsstreit an einem Berliner Gymnasium in Bezug auf das muslimische Gebet in Schulräumen.

Schura Niedersachsen möchte einen Anstoß zu Modellprojekten geben und würde sich freuen, wenn dieser Gedanke von der Landesintegrations- und der Schulverwaltung in Kooperation mit der Religionsgemeinschaft aufgegriffen würde.

„Mit Vertrauen auf Klassenfahrt“ ist oben getitelt. Schura Niedersachsen erreichen immer wieder solche und ähnliche Meldungen von Eltern und Schulkindern über erfahrene Diskriminierungen; Meldungen, die erkennen lassen, dass mancherorts fehlende oder mangelnde Kenntnis oder gar Unverständnis vorliegen. Diese Vorgänge sollen hier nicht bewertet oder vertieft werden. Es ist aber verständlich, dass vor solchem Erfahrungshintergrund gläubige Familien sich schwer tun, ihre Kinder vorbehaltlos mehrtägig von sich zu lassen; hier hätte das Wort ,anvertrauen“ stehen müssen, aber genau darum geht es hier.

Zielgruppe interkulturellen Trainings sind insbesondere Mitarbeiterinnen und Mitarbeiter in Schule, Kindergarten, Jugendherberge oder Landschulheim. Schura Niedersachsen hat heute noch kein konkretes Maßnahmekonzept. Einen vorbildlichen Beitrag wird das Projekt „Religionssensible Schulkultur“ der AlbertSchweitzer-Schule in Hannover liefern. Kern und Ergebnis der Bemühungen müssen alle Maßnahmen sein, die Vertrauen nachhaltig begründen.

\section{4. „Gott im Himmel, glauben nach ihrem heiligen Koran die Mohammedaner auch an Moses?،}

Diese Frage ist hypothetisch; sie verdichtet holzschnittartig die Position des nichtmuslimischen Fragenden hierzulande, ausgedrückt in Denkmustern, die erlebter deutscher christlicher Tradition entspringen. Wir stehen am Anfang islamischer Religionspädagogik in Deutschland, auch der Produktion von Curricula und Schulbüchern zum islamischen Religionsunterricht sowie zur universitären Bildung in islamischen Studien. Schon heute ist eine Begriffsvielfalt zur Beschreibung islamisch-theologischer und -gesellschaftlicher Inhalte in deutschsprachiger Lite- 
ratur zu beklagen. Bevor sich Fehlübersetzungen und -konnotationen in Schrift und im Gedächtnis nachfolgender Schüler- und Studentengenerationen verfestigen, benötigen wir eine von Muslimen zu leistende wissenschaftliche Inventur.

Einige Fundament- und Bausteine sowie die Motivation zu diesem Diskurs werden hier vorgestellt. Es bedarf in jedem Fall der Abstimmung mit der Religionsgemeinschaft, auch um am Ende die heterogene Verwendung von arabischen, deutschen und eingedeutschten Ausdrücken und Namen nicht auf dem Rücken der Kinder auszutragen.

Warum übersetzen wir nicht Synode (Zusammenkunft) oder Pastor (Hirte) ins Deutsche? Ist Schura Synode? Die Frage weist auf die unhinterfragte Gebräuchlichkeit der griechischstämmigen Fremdworte hin und lässt erahnen, dass sich die Gesellschaft schwer tun wird und es dennoch hinnehmen muss, mit der Zuwanderung - quantitativ tatsächlich von Religion - auch deren Begriffswelt als - hier arabische - Fremdworte zu ,integrieren“. Andernfalls würden deutschsprachige Adäquate wegen der i.d.R. christlichen Konnotation einer inhaltlichen und damit verständnismäßigen Schieflage unterworfen.

Dazu gehören dezidierte Positionen gegen eine äußerliche Verchristlichung im islamischen Religionsunterricht: Gott ist nicht im Himmel! Gott, jenseits von Zeit und Raum, hat keinen Ort; Muslime „glauben“ nicht, sie bezeugen aufgrund Vertrauens und bestmöglichen Wissens, dessen Erlangung religiöse Pflicht ist und für das das Individuum unmittelbar vor Gott verantwortlich ist. Soll es im Islam hierzulande Allah oder Gott heißen? Hier sind sich auch die Muslime nicht einig. Koran oder Qur'an: wieweit kann Eindeutschung gehen, ohne den mit dem Arabischen verknüpften Assoziationsinhalt zu verlieren? Im Islam ist nichts „heilig“, die abendländische Trennung in profan und heilig wird nicht nachvollzogen. Nur die Selbstbezeichnung (Muslime) kann authentisch sein, nicht der ältere deutsche Begriff „Mohammedaner“.

Muslime bezeugen, dass Musa (a.s.) Gottes Gesandter war, sie bedürfen weder der hebräischen (Moshe) noch der griechischen Namensform (Moses). Können Jesus (lateinisch für aramäisches Jeshua), Christus und Isa (a.s.) synonym verwendet werden? Der Qur'an transportiert zur Frage der Identität von Jesus/Isa (a.s.) und $\mathrm{zu}$ dem, was er wirklich sagte und tat, deutlich andere Inhalte als die griechisch-europäischen Traditionen und dogmatischen Positionen. Sind Jesus, Moses, Abraham (nur) deutsche Namen? Mit seinem Vater spricht der Schüler am Opferfest über Ibrahim und Gottes Forderung, ihm seinen Sohn (Ismael) zu opfern; und er vergisst nicht, aleihi salam (a.s., Friede sei mit ihm) zu sagen. In der Schule lernt er über Abraham: ein Identitätskonflikt? Ist die Kultusverwaltung befugt, die Sprachform „Jesus“ für den islamischen Religionsunterricht festzulegen?

Weitere Begriffe seien beispielhaft erwähnt, für die es keine deutschen Entsprechungen gibt bzw. deren aus dem christlichen Ritus entlehnte deutsche, griechische oder lateinische Begriffe den islamischen Sinngehalt nicht (treffend) wie- 
dergeben: Iman Glaube, Zakah Armensteuer, Salah Gebet, Du'a Gebet, Rahma Barmherzigkeit, Islam Hingabe, Ayah Wunderzeichen, Vers, Kufr-Kafir UnglaubeUngläubiger, Djibad heiliger Krieg, Tauba Reue, Isnad. Weite Bereiche des rituellen islamischen Rechts sind ohne deutsche Begriffsäquivalente oder - wie beim letztgenannten Begriff - aus dem frühen christlichen Kontext der Genese des Kanons völlig unbekannt.

Grundlage des islamischen Religionsunterrichts muss ein islamisch-theologisch korrektes Begriffsinventar für den Gebrauch innerhalb der deutschen Sprache sein. Es zeichnet sich ab, dass - auch auf Grundlage erfolgreicher Ansätze im englischsprachigen Raum - Forschungsarbeit zu leisten ist, Arbeit, deren alsbaldig zu erzielende Ergebnisse im deutschsprachigen Raum möglichst einheitlich, also auch jenseits des föderalen Prinzips, umgesetzt und durch Erklärungen islamischer Autoritäten und Repräsentanz hinreichend verbindlich werden müssen. Schura Niedersachsen bietet der Universität Osnabrück und der Lehrplankommission beim MK ihre Mitarbeit an. 


\section{Die Autorinnen und Autoren}

Avni Altiner, Vorsitzender der Schura e.V. Niedersachsen

Heidemarie Ballasch, Regierungsdirektorin im niedersächsischen Kultusministerium

Kinan Darwisch, Promotionsstudent an der Sozialwissenschaftlichen Fakultät der Georg-August-Universität Göttingen

Gerdien Jonker, Dr., wissenschaftliche Mitarbeiterin am Georg-Eckert-Institut für Schulbuchforschung Braunschweig

Katja Koch, Prof. Dr., Professorin für Schulpädagogik, Institut für Erziehungswissenschaft, Technische Universität Braunschweig

Ali Özgur Özdil, Dr. des., Direktor des Islamischen Wissenschafts- und Bildungsinstitut e.V. Hamburg

Betram Schmitz, PD Dr. Dr. , Institut für Theologie und Religionswissenschaft, Leibniz-Universität Hannover 
Nazife Toklu, Dipl. Sozialpädagogin, Dipl. Sozialarbeiterin, Sozialberaterin und Organisatorin für Integrationskurse bei verikom - Verbund für interkulturelle Kommunikation und Bildung e.V. - Hamburg.

Haci-Halil Uslucan, PD Dr., Vertretung der Professur für Pädagogische Psychologie, Fakultät für Geistes- und Sozialwissenschaften, Helmut-SchmidtUniversität Hamburg

Firouz Vladi, Bildungsreferent im Vorstand der Schura Niedersachsen. und Vertreter der Schura Niedersachsen am Runden Tisch im niedersächsischen Kultusministerium 
An niedersächsischen Schulen lernen schätzungsweise 45.000 muslimische Schülerinnen und Schüler. Ihre Integration in das deutsche Schul- und Gesellschaftssystem verlangt komplexe pädagogische und politische Maßnahmen, aber auch eine aktive und verantwortungsbewusste Mitarbeit der muslimischen Organisationen. Um diesem Umstand Rechnung zu tragen führte das Land Niedersachsen im Schuljahr 2003/2004 den Schulversuch „Islamischer Religionsunterricht" in Zusammenarbeit mit führenden muslimischen Organisationen ein.

Der vorliegende Band fasst u.a. die Ergebnisse eines Zusammentreffens von Wissenschaftlern verschiedener Disziplinen sowie von Vertretern des Landes Niedersachsens und der Schura Niedersachsen e.V. auf der Tagung „Dimensionen religiöser Erziehung muslimischer Kinder in Niedersachsen" in Göttingen zusammen. Dabei wird über historische, schulische, politische sowie pädagogische Aspekte auf die Komplexität der Thematik, aber auch die Relevanz eines islamischen Religionsunterrichts und dessen Potential aufmerksam gemacht. 